Supporting Information

for

\title{
Measurement of Heme Ruffling Changes in MhuD Using
}

\section{UV-vis Spectroscopy}

Amanda B. Graves, Max T. Graves, Matthew D. Liptak

Department of Chemistry, University of Vermont, Burlington, Vermont, 05405, United States

\section{Contents}

Supplementary Experimental Section

S3-9

Supplementary Results

S9-11

Table S1 W66F MhuD Mutagenic Primer Sequence

S12

Table S2 W66A MhuD Mutagenic Primer Sequence

S12

Table S3 W66F MhuD Gene Sequence

S12

Table S4 W66A MhuD Gene Sequence

S13

Table S5 Secondary Structure Analysis

S13

Table S6 NSD Program Test Results

S14-17

Figure S1 W66F MhuD SDS-PAGE Gel

S17

Figure S2 W66A MhuD SDS-PAGE Gel

S18 
$\begin{array}{llr}\text { Figure S3 } & \text { Angle Constraint used for DFT Models } & \text { S18 }\end{array}$

$\begin{array}{lll}\text { Figure S4 Heme Degradation by WT MhuD with Catalase } & \text { S19 }\end{array}$

Figure S5 Heme Degradation by W66F MhuD with Catalase $\quad$ S19

Figure S6 Heme Degradation by W66A MhuD with Catalase $\quad$ S20

$\begin{array}{llr}\text { Figure S7 Non-enzymatic Heme Degradation } & \text { S20 }\end{array}$

$\begin{array}{lll}\text { Figure S8 UV CD Spectroscopy } & \text { S21 }\end{array}$

Figure S9 $\quad d$ Orbital Occupation of Singly Occupied Molecular Orbital $\quad$ S21

$\begin{array}{llr}\text { Figure S10 } & \text { PBE0/TZVP-computed Valence Orbitals } & \text { S22 }\end{array}$

Cartesian Coordinates for all computational models $\quad$ S22-76 


\section{Supplementary methods.}

CD Spectroscopy. Circular dichroism (CD) spectra of the wild type (WT), W66F, and W66A forms of cyanide-inhibited (MhuD-heme- $\mathrm{CN}$ ) were acquired in the $\mathrm{UV}$ region of the spectrum to investigate the overall fold of each variant. Samples of MhuD-heme-CN in $10 \mathrm{mM}$ potassium phosphate (KPi) pH 7.4 were loaded into quartz cuvettes, and the UV CD spectra from 240 to 190 $\mathrm{nm}$ were acquired on a Jasco J-815 spectropolarimeter using a scanning speed of $20 \mathrm{~nm} / \mathrm{min}$, a bandwidth of $1 \mathrm{~nm}$, a digital integration time of $8 \mathrm{~s}$, and a data pitch of $0.5 \mathrm{~nm}$. The spectra were analyzed using the SELCON3 program of the CDPro software package with IBasis=4 in order to evaluate the secondary structure of each variant. ${ }^{1-5}$

Normal Coordinate Structural Decomposition. In order to analyze the out-of-plane distortions of the heme structures presented in this work in terms of their displacements from $D_{4 h}$ symmetry along low-energy vibrational normal modes, a new version of the normal-coordinate structural decomposition program, originally developed by Shelnutt and co-workers, ${ }^{6}$ was written. This was necessary because, unfortunately, the original program is no longer available on the internet. The new program features a more flexible input format, and is available for use on the corresponding author's website. Based upon the compelling correlations between out-of-plane deformation type and function, ${ }^{7}$ and the lack of compelling correlations between in-plane deformation type and function, the new program only provides out-of-plane normal coordinate displacements. Similar to the original program, the new program transforms the out-of-plane distortions of the 24 atoms of the porphyrin $\pi$ system into a sum of deformations along six normal modes. The first step of the program aligns the observed porphyrin coordinates $\left(\boldsymbol{S}_{o b s}\right)$ with a set of reference coordinates $\left(\boldsymbol{S}_{r e f}\right)$ where the reference coordinates are an ideal, planar porphyrin so the displacement of each individual atom in the observed structure can be determined. $\boldsymbol{S}_{r e f}$ was chosen to be the same 
energy minimized structure as used in the original NSD program, copper(II) porphine, and the atomic coordinates are found in Table S1 of Shelnutt and coworkers. ${ }^{6}$ The 24 x 3 matrix was written as a 72-element column vector whose first three elements are the $\mathrm{x}, \mathrm{y}$, and $\mathrm{z}$ atomic position coordinates of the first atom, then the next three elements are the $\mathrm{x}, \mathrm{y}$, and $\mathrm{z}$ coordinates of the second atom, etc., where the z-values are all 0 to ensure the reference structure is completely planar. $\boldsymbol{S}_{r e f}$ was subtracted from $\boldsymbol{S}_{\text {obs }}$ to provide the out-of-plane distortion for the observed system $\left(\boldsymbol{D}_{o b s}\right)$ :

$$
\boldsymbol{D}_{o b s}=\boldsymbol{S}_{o b s}-\boldsymbol{S}_{\text {ref }}
$$

However, before $\boldsymbol{D}_{o b s}$ is computed, the coordinates for $\boldsymbol{S}_{o b s}$ were properly aligned with the reference structure as described below.

The center-of-coordinate (non-weighted center-of-mass) is computed from the position coordinates of all the atoms in the observed molecule by:

$$
\vec{r}_{c m}=\sum_{i=1}^{N} \vec{r}_{i}
$$

where $\vec{r}_{i}$ is the coordinate of each individual atom and $\mathrm{N}$ is the total number of atoms. $\vec{r}_{c m}$ was subtracted from each individual coordinate, putting the observed center-of-coordinate at the origin.

The coordinates of the observed molecule were rotated such that the mean plane (or "best fit plane") of the reference molecule (reference plane) and the mean plane of the observed molecule are co-planar, each with an associated unit normal vector. The coordinate system was chosen such that the mean plane for the reference molecule is defined by the unit normal $\hat{n}=\hat{z}$. The unit normal for the mean plane of the observed molecule, $\hat{m}=m_{x} \hat{x}+m_{y} \hat{y}+m_{z} \hat{z}$, was determined via consideration of the residual sum of squares: 


$$
\Delta_{z}=\sum_{i=1}^{N}\left(\vec{m} \cdot \vec{r}_{i}^{o b s}\right)^{2}
$$

The residual sum of squares is a measure of the discrepancy between the observed coordinates and the reference positions. ${ }^{8}$ When the mean planes are co-planar, the residual sum of squares will be minimized, thus finding the proper $\vec{m}$ is an extremization problem solved by taking the length of $\vec{m}$ as a stationary condition and introducing a Lagrange multiplier $\eta$.

$$
F=\sum_{i=1}^{N}\left(\vec{m} \cdot \vec{r}_{i}^{o b s}\right)^{2}-\eta\left(m_{x}^{2}+m_{y}^{2}+m_{z}^{2}\right)
$$

The equation is extremized when the following conditions are fulfilled:

$$
\frac{1}{2} \frac{\partial}{\partial m_{j}} F=\frac{\partial}{\partial m_{j}} \sum_{i=1}^{N}\left(\vec{m} \cdot \vec{r}_{i}^{o b s}\right)^{2}-\eta m_{j}=0, j=x, y, z
$$

This was represented, in matrix notation, as the eigenvalue problem

$$
\boldsymbol{U} \vec{m}=\eta \vec{m}
$$

where $\mathbf{U}$ is given by

$$
\boldsymbol{U}=\left[\begin{array}{lll}
\vec{S}_{o b s}^{x} \vec{S}_{o b s}^{x} & \vec{S}_{o b s}^{x} \vec{S}_{o b s}^{y} & \vec{S}_{o b s}^{x} \vec{S}_{o b s}^{z} \\
\vec{S}_{o b s}^{y} \vec{S}_{o b s}^{x} & \vec{S}_{o b s}^{y} \vec{S}_{o b s}^{y} & \vec{S}_{o b s}^{y} \vec{S}_{o b s}^{z} \\
\vec{S}_{o b s}^{z} \vec{S}_{o b s}^{x} & \vec{S}_{o b s}^{z} \vec{S}_{o b s}^{y} & \vec{S}_{o b s}^{z} \vec{S}_{o b s}^{z}
\end{array}\right]
$$

and $\vec{S}_{o b s}^{x}$ (for example) is a 24-element column vector containing the x-coordinates of the observed molecule. The unit normal $\widehat{m}$ was found by constructing this matrix and computing its eigenvectors. Once normalized, the eigenvector with the lowest corresponding eigenvalue was chosen as the unit normal $\widehat{m}$. 
Upon determining $\widehat{m}$, the axis of rotation to align the observed coordinates with the reference coordinates was computed with the unit vector that points along that axis:

$$
\widehat{u}=\frac{\widehat{m} \times \hat{n}}{|\widehat{m} \times \hat{n}|}
$$

This unit vector defines the axis of rotation used in order to align the mean plane of the observed coordinates co-planar with the reference plane. The observed coordinates were chosen to lie in the $x y$-plane, and the unit vector about which to rotate becomes

$$
\hat{u}=\frac{m_{y} \hat{x}-m_{x} \hat{y}}{\sqrt{m_{x}^{2}+m_{y}^{2}}}
$$

Then a rotation around $\hat{u}$ by an angle

$$
\beta=\cos ^{-1} m_{z}
$$

was performed using the Rodriguez Rotation Formula given by:

$$
\begin{gathered}
\boldsymbol{R}(\beta)= \\
{\left[\begin{array}{ccc}
\cos \beta+u_{x}^{2}(1-\cos \beta) & u_{x} u_{y}(1-\cos \beta)-u_{z} \sin \beta & u_{x} u_{z}(1-\cos \beta)+u_{y} \sin \beta \\
u_{x} u_{y}(1-\cos \beta)+u_{z} \sin \beta & \cos \beta+u_{y}^{2}(1-\cos \beta) & u_{y} u_{z}(1-\cos \beta)-u_{x} \sin \beta \\
u_{x} u_{z}(1-\cos \beta)-u_{y} \sin \beta & u_{y} u_{z}(1-\cos \beta)+u_{x} \sin \beta & \cos \beta+u_{z}^{2}(1-\cos \beta)
\end{array}\right]}
\end{gathered}
$$

Since $u_{z}=0$, this rotation matrix is simplified to

$$
R(\beta) \rightarrow\left[\begin{array}{ccc}
\cos \beta+u_{x}^{2}(1-\cos \beta) & u_{x} u_{y}(1-\cos \beta) & u_{y} \sin \beta \\
u_{x} u_{y}(1-\cos \beta) & \cos \beta+u_{y}^{2}(1-\cos \beta) & -u_{x} \sin \beta \\
-u_{y} \sin \beta & u_{x} \sin \beta & \cos \beta
\end{array}\right]
$$

In order to rotate the observed coordinates' mean plane into the reference plane, the kth atom's observed coordinates were rotated to compute $\vec{r}_{k}^{\text {obs,coplanar }}=\boldsymbol{R}(\beta) \cdot \vec{r}_{k}^{\text {obs }}$ (herein $\vec{r}_{k}^{o b s}$ ). This 
operation was done for all atoms and the resulting observed coordinates' mean plane lies in the $x y$ plane. The $\vec{S}_{\text {obs }}$ vectors refer to those whose coordinates' mean plane has been aligned with that of the reference structure.

The second step for aligning the observed structure with the reference structure was to rotate the observed coordinates around the z-axis by an angle $\theta$. The sum of the residual sum of squares was extremized to find the desired quantity, the angle $\theta$. The residual sum of squares for this rotation is given by

$$
\Delta_{x y}=\sum_{i=1}^{N}\left(\boldsymbol{R}(\theta) \cdot \vec{r}_{i}^{o b s}-\vec{r}_{i}^{r e f}\right)^{2},
$$

where $\boldsymbol{R}(\theta)$ is the rotation matrix

$$
\boldsymbol{R}(\theta)=\left[\begin{array}{ccc}
\cos (\theta) & \sin (\theta) & 0 \\
-\sin (\theta) & \cos (\theta) & 0 \\
0 & 0 & 1
\end{array}\right]
$$

The residual sum of squares must once again be minimized, with the following condition met:

$$
\frac{1}{2} \frac{d}{d \theta} \Delta_{x y}=\sum_{i=1}^{N}\left(\boldsymbol{R}(\theta) \cdot \vec{r}_{i}^{o b s}-\vec{r}_{i}^{r e f}\right)\left(\frac{d \boldsymbol{R}(\theta)}{d \theta} \vec{r}_{i}^{o b s}\right)=0
$$

This had the solution

$$
\theta+n \pi=\arctan \left(\frac{\vec{S}_{r e f}^{x} \vec{S}_{o b s}^{y}-\vec{S}_{r e f}^{y} \vec{S}_{o b s}^{x}}{\vec{S}_{r e f}^{x} \vec{S}_{o b s}^{x}+\vec{S}_{r e f}^{y} \vec{S}_{o b s}^{y}}\right), n \in \mathbb{Z}
$$

Since $0 \leq \theta \leq 2 \pi$, there is only one minimum and one maximum. Taking the second derivative of the previous equation and rearranging yielded the following condition for the minimum value of $\theta$ : 


$$
\left(\vec{S}_{r e f}^{x} \cdot \vec{S}_{o b s}^{y}-\vec{S}_{r e f}^{y} \cdot \vec{S}_{o b s}^{x}\right) \cos (\theta)+\left(\vec{S}_{r e f}^{x} \cdot \vec{S}_{o b s}^{x}+\vec{S}_{r e f}^{y} \cdot \vec{S}_{o b s}^{y}\right) \sin (\theta)>0
$$

These conditions determine the value of $\theta$ to rotate the coordinates to align the observed and reference structures.

The distortion coordinates are represented as a linear combination of the harmonic eigenvectors:

$$
\boldsymbol{D}_{o b s}=\sum_{\Gamma, m} d_{m}^{\Gamma} \widehat{\boldsymbol{D}}_{m}^{\Gamma} \cos \left(2 \pi c \tilde{v}_{m}^{\Gamma} \mathrm{t}+\phi_{\mathrm{m}}^{\Gamma}\right)
$$

where $d_{m}^{\Gamma}$ is the magnitude of the displacement along the normal coordinate contributed from the $\mathrm{m}^{\text {th }}$ mode of symmetry type $\Gamma$ (e.g. $a_{1 u}, a_{2 u}, b_{1 u}$, etc...), $\tilde{v}_{m}^{\Gamma}$ is the vibrational frequency of that normal mode, $c$ is the speed of light, and $\widehat{\boldsymbol{D}}_{m}^{\Gamma}$ are the normal displacement eigenvectors. These displacement eigenvectors were treated as 72-element column vectors (ordered the same as $\boldsymbol{D}_{o b s}$ ), with values of 0 entered for the $\mathrm{x}$ - and $\mathrm{y}$-coordinates. In the static case (no time dependence), $t=$ 0 , creates an initial phase shift $\phi_{\mathrm{m}}^{\Gamma}=0$, which leaves the observed distortion vectors in the form:

$$
\boldsymbol{D}_{o b s}=\sum_{\Gamma, m} d_{m}^{\Gamma} \widehat{\boldsymbol{D}}_{m}^{\Gamma}
$$

The normal displacement eigenvectors for the three lowest-frequency normal modes $(\mathrm{m}=1,2,3)$ are given in Tables S3 and S4 in Jentzen et. al. ${ }^{6}$ The magnitudes of these distortions, $d_{m}^{\Gamma}$, were computed as:

$$
d_{m}^{\Gamma}=\sum_{k=1}^{N_{\Gamma}} \bar{B}_{m k}^{\Gamma} P_{k}^{\Gamma}
$$

with $\bar{B}_{m k}^{\Gamma}$ being the matrix element from the $m$ th row and $k$ th column of the matrix $\overline{\boldsymbol{B}}^{\Gamma}=\left(\boldsymbol{B}^{\Gamma}\right)^{-1}$, where $B_{m k}^{\Gamma}=\widehat{D}_{m}^{\Gamma \prime} \cdot \widehat{D}_{k}^{\Gamma}$, and $P_{k}^{\Gamma}=\widehat{D}_{m}^{{ }^{\prime}} \cdot \boldsymbol{D}_{o b s}$. For clarity, an example of the $\boldsymbol{B}^{B 1 u}$ matrix is provided: 


$$
\boldsymbol{B}^{B 1 u}=\left[\begin{array}{lll}
\widehat{D}_{1}^{\prime B 1 u} \cdot \widehat{D}_{1}^{B 1 u} & \widehat{D}_{1}^{\prime B 1 u} \cdot \widehat{D}_{2}^{B 1 u} & \widehat{D}_{1}^{\prime B 1 u} \cdot \widehat{D}_{3}^{B 1 u} \\
\widehat{D}_{2}^{\prime B 1 u} \cdot \widehat{D}_{1}^{B 1 u} & \widehat{D}_{2}^{\prime B 1 u} \cdot \widehat{D}_{2}^{B 1 u} & \widehat{D}_{2}^{\prime B 1 u} \cdot \widehat{D}_{3}^{B 1 u} \\
\widehat{D}_{3}^{\prime B 1 u} \cdot \widehat{D}_{1}^{B 1 u} & \widehat{D}_{3}^{\prime B 1 u} \cdot \widehat{D}_{2}^{B 1 u} & \widehat{D}_{3}^{\prime B 1 u} \cdot \widehat{D}_{3}^{B 1 u}
\end{array}\right]
$$

and therefore $B_{21}^{\Gamma}=\widehat{D}_{2}^{\prime B 1 u} \cdot \widehat{D}_{1}^{B 1 u}$. Then,

$$
d_{1}^{\mathrm{B} 1 \mathrm{u}}=\sum_{k=1}^{N_{\Gamma}=24} \bar{B}_{1 k}^{\mathrm{B} 1 \mathrm{u}} P_{k}^{\mathrm{B} 1 \mathrm{u}}
$$

is the displacement of the $1^{\text {st }}$ frequency mode of the $b_{1 u}$ (ruffling) irreducible representation. This value is reported as the displacement from the minimal basis. The displacement value from the extended basis is the sum of the $m=1,2,3$ displacement contributions.

\section{Supplementary results.}

CD Spectroscopy. CD spectra of WT, W66F, and W66A MhuD-heme-CN were acquired in the UV region and analyzed to determine whether the dependence of the MhuD-catalyzed heme degradation rate on the identity of residue 66 could be attributed to polypeptide structural changes. This experiment is an excellent probe of the overall protein fold as the fractional contributions of $\alpha$-helices and $\beta$-sheets to the polypeptide secondary structure can be determined from careful analysis of the spectroscopic data. ${ }^{9}$ All three CD spectra have similar intensity negative bands at $209 \mathrm{~nm}$, but there are small differences between variants for the shoulder observed at $222 \mathrm{~nm}$ and the positive peak detected at $191 \mathrm{~nm}$ in WT MhuD-heme-CN (Figure S8). In addition, the zerocrossing point of the spectrum shifts from $198 \mathrm{~nm}$ in WT and W66A MhuD-heme-CN to $197 \mathrm{~nm}$ in the W66F variant. In order to determine whether these differences have significant structural implications, all three spectra were analyzed using the CDPro software package. ${ }^{1,2}$ The fractional contributions of $\alpha$-helices and $\beta$-sheets to the MhuD-heme- $\mathrm{CN}$ secondary structure in solution, as detected using CD spectroscopy, are 30\% less than those observed in the solid state by X-ray 
crystallography (PDB ID 4NL5, Table S5). ${ }^{10}$ This provides an estimate of the systematic error for $\mathrm{CD}$ analysis of the MhuD-heme-CN secondary structure, which is similar to the RMSD error for the SELCON3 algorithm. The ratio of $\alpha$-helices to $\beta$-sheets deduced by X-ray crystallography and CD spectroscopy is nearly identical, suggesting that the protein fold in solution is similar to that detected by crystallography. Analysis of the CD data for W66F and W66A MhuD-heme-CN indicates that these substitutions may increase the $\alpha$-helical content and decrease the $\beta$-sheet content, but the changes are smaller than the estimated systematic error. Thus, all three forms of MhuD-heme-CN are likely to have protein folds similar to that of the available crystal structure.

Normal structural decomposition program. The consistency of the old and new NSD program output was assessed by comparison of the out-of-plane heme distortions for a library of 42 heme structures. ${ }^{6}$ The maximum difference between the normal coordinate distortions calculated by both programs was $0.01 \AA$, and 289 of the 294 values had differences of less than $0.005 \AA$ (Table S6). Based upon this analysis, and the errors inherent to the DREIDING II force field used to generate the normal mode vectors, ${ }^{10}$ NSD program output is rounded to the nearest $0.01 \AA$.

\section{Supplementary references.}

1. Sreerama, N., and Woody, R. W. A Self-consistent Method for the Analysis of Protein Secondary Structure from Circular Dichroism. Anal. Biochem. 1993, 209, 32-44.

2. Sreerama, N., and Woody, R. W. Estimation of Protein Secondary Structure from Circular Dichroism Spectra: Comparison of CONTIN, SELCON, and CDSSTR Methods with an Expanded Reference Set. Anal. Biochem. 2000, 287, 252-260.

3. Sreerama, N., Venyaminov, S. Y., and Woody, R. W. Estimation of the Number of Alpha-helical and Beta-strand Segments in Proteins Using Circular Dichroism Spectroscopy. Protein Sci. 1999, 8, 370 380.

4. Sreerama, N., and Woody, R. W. Poly(pro)II Helices in Globular Proteins: Identification and Circular Dichroic Analysis. Biochemistry. 1994, 33, 10022-10025.

5. Johnson, W. C. Analyzing Protein Circular Dichroism Spectra for Accurate Secondary Structures. Proteins. 1999, 35, 307-312.

6. Jentzen, W., Song, X. Z., and Shelnutt, J. A. Structural Characterization of Synthetic and Proteinbound Porphyrins in Terms of the Lowest-Frequency Normal Coordinates of the Macrocycle. J. Phys. Chem. B. 1997, 101, 1684-1699. 
7. Jentzen, W., Ma, J. G., and Shelnutt, J. A. Conservation of the Conformation of the Porphyrin Macrocycle in Hemoproteins. Biophys. J. 1998, 74, 753-763.

8. Schomaker, G., Waser, J., Marsh, R. E., and Bergman, G. To Fit a Plane or Line to a Set of Points by Least Squares. Acta Crystallogr. 1959, 12, 600-604.

9. Greenfield, N. J. Using Circular Dichroism Spectra to Estimate Protein Secondary Structure. Nat. Proto. 2006, 1, 2876-2890.

10. Graves, A. B., Morse, R. P., Chao, A., Iniguez, A., Goulding, C. W., and Liptak, M. D. Crystallographic and Spectroscopic Insights into Heme Degradation by Mycobacterium tuberculosis MhuD. Inorg. Chem. 2014, 53, 5931-5940. 
Table S1. W66F MhuD mutagenic primer sequence

\begin{tabular}{|l|c|}
\hline Primer & \multicolumn{1}{|c|}{ Sequence } \\
\hline Forward & $5^{\prime}$ - GAAGCATTCCAGGCGTTCGCAAACGGGCCC-3' \\
\cline { 2 - 2 } Reverse & 5'- GGGCCCGTTTGCGAACGCCTGGAATGCTTC -3' \\
\hline
\end{tabular}

Table S2. W66A MhuD mutagenic primer sequence

\begin{tabular}{|l|c|}
\hline Primer & Sequence \\
\hline Forward & 5'-GCATTCCAGGCGGCGGCAAACGGGCCC-3' \\
\cline { 2 - 2 } Reverse & 5'-GGGCCCGTTTGCCGCCGCCTGAAGTGC-3' \\
\hline
\end{tabular}

Table S3. W66F MhuD gene sequence

\begin{tabular}{|c|c|c|c|c|c|c|c|c|}
\hline $\begin{array}{c}\text { M1 } \\
\text { ATG }\end{array}$ & $\begin{array}{c}\mathrm{P} 2 \\
\mathrm{CCA}\end{array}$ & $\begin{array}{c}\text { V3 } \\
\text { GTG }\end{array}$ & $\begin{array}{c}\text { V4 } \\
\text { GTG }\end{array}$ & $\begin{array}{c}\mathrm{K} 5 \\
\mathrm{AAG}\end{array}$ & $\begin{array}{c}\text { I6 } \\
\text { ATC }\end{array}$ & $\begin{array}{c}\text { N7 } \\
\text { AAC }\end{array}$ & $\begin{array}{c}\text { A8 } \\
\text { GCA }\end{array}$ & $\begin{array}{c}\text { I9 } \\
\text { ATC }\end{array}$ \\
\hline E10 & V11 & P12 & A13 & G14 & A15 & G16 & P17 & E18 \\
\hline GAG & GTG & $\mathrm{CCC}$ & GCC & GGC & GCT & GGC & $\mathrm{CCC}$ & GAG \\
\hline L19 & E20 & K21 & $\mathrm{R} 22$ & F23 & A24 & $\mathrm{H} 25$ & $\mathrm{R} 26$ & A27 \\
\hline CTG & GAG & $\mathrm{AAG}$ & CGG & TTC & GCT & CAC & CGC & GCG \\
\hline $\mathrm{H} 28$ & A29 & V30 & E31 & N32 & S33 & P34 & G35 & F36 \\
\hline CAC & GCG & GTC & GAG & AAC & TCC & $\mathrm{CCG}$ & GGT & TTC \\
\hline L37 & G38 & F39 & Q40 & L41 & L42 & R43 & $\mathrm{P} 44$ & V45 \\
\hline CTC & GGC & TTT & $\mathrm{CAG}$ & CTG & TTA & CGT & CCG & GTC \\
\hline K46 & G47 & E48 & E49 & R50 & Y51 & F52 & V53 & V54 \\
\hline AAG & GGT & GAA & GAA & CGC & TAC & TTC & GTG & GTG \\
\hline T55 & H56 & W57 & E58 & S59 & D60 & E61 & A62 & F63 \\
\hline ACA & CAC & TGG & GAG & TCC & GAT & GAA & GCA & TTC \\
\hline Q64 & A65 & F66 & A67 & N68 & G69 & P70 & A71 & $\mathrm{I} 72$ \\
\hline CAG & GCG & TTC & GCA & $\mathrm{AAC}$ & GGG & $\mathrm{CCC}$ & GCC & ATC \\
\hline A73 & A74 & H75 & A76 & G77 & $\mathrm{H} 78$ & R79 & A80 & N81 \\
\hline GCA & GCC & CAT & $\mathrm{GCC}$ & GGA & CAC & CGG & GCC & $\mathrm{AAC}$ \\
\hline P82 & V83 & A84 & T85 & G86 & A87 & S88 & L89 & L90 \\
\hline $\mathrm{CCC}$ & GTG & GCG & $\mathrm{ACC}$ & GGT & GCT & TCG & CTG & CTG \\
\hline E91 & F92 & E93 & V94 & V95 & L96 & D97 & V98 & G99 \\
\hline GAA & TTC & GAG & GTC & GTG & CTT & GAC & GTC & GGT \\
\hline G100 & $\mathrm{T} 101$ & G102 & K103 & $\mathrm{T} 104$ & A105 & G106 & G107 & V108 \\
\hline GGG & $\mathrm{ACC}$ & GGC & AAG & ACT & GCA & GGA & GGT & GTA \\
\hline P109 & R110 & G111 & K112 & L113 & A114 & A115 & A116 & L117 \\
\hline CCA & CGA & GGT & AAG & CTT & GCG & GCC & GCA & CTC \\
\hline E118 & H1 19 & H120 & H121 & H122 & H123 & H124 & & \\
\hline GAG & CAC & CAC & CAC & CAC & CAC & CAC & & \\
\hline
\end{tabular}


Table S4. W66A MhuD gene sequence

\begin{tabular}{|c|c|c|c|c|c|c|c|c|}
\hline $\begin{array}{c}\text { M1 } \\
\text { ATG }\end{array}$ & $\begin{array}{c}\text { P2 } \\
\text { CCA }\end{array}$ & $\begin{array}{c}\text { V3 } \\
\text { GTG }\end{array}$ & $\begin{array}{c}\text { V4 } \\
\text { GTG }\end{array}$ & $\begin{array}{c}\text { K5 } \\
\text { AAG }\end{array}$ & $\begin{array}{c}\text { I6 } \\
\text { ATC }\end{array}$ & $\begin{array}{c}\mathrm{N} 7 \\
\mathrm{AAC}\end{array}$ & $\begin{array}{c}\text { A8 } \\
\text { GCA }\end{array}$ & $\begin{array}{c}\text { I9 } \\
\text { ATC }\end{array}$ \\
\hline E10 & V11 & $\mathrm{P} 12$ & A13 & G14 & A15 & G16 & P17 & E18 \\
\hline GAG & GTG & $\mathrm{CCC}$ & GCC & GGC & GCT & GGC & $\mathrm{CCC}$ & GAG \\
\hline L19 & E20 & K21 & $\mathrm{R} 22$ & F23 & A24 & $\mathrm{H} 25$ & $\mathrm{R} 26$ & A27 \\
\hline CTG & GAG & AAG & CGG & TTC & GCT & CAC & CGC & GCG \\
\hline $\mathrm{H} 28$ & A29 & V30 & E31 & N32 & S33 & P34 & G35 & F36 \\
\hline CAC & GCG & GTC & GAG & $\mathrm{AAC}$ & TCC & $\mathrm{CCG}$ & GGT & TTC \\
\hline L37 & G38 & F39 & Q40 & L41 & L42 & $\mathrm{R} 43$ & $\mathrm{P} 44$ & V45 \\
\hline CTC & GGC & TTT & CAG & CTG & TTA & CGT & CCG & GTC \\
\hline K46 & G47 & E48 & E49 & R50 & Y51 & F52 & V53 & V54 \\
\hline AAG & GGT & GAA & GAA & CGC & TAC & TTC & GTG & GTG \\
\hline T55 & H56 & W57 & E58 & S59 & D60 & E61 & A62 & F63 \\
\hline ACA & CAC & TGG & GAG & TCC & GAT & GAA & GCA & TTC \\
\hline Q64 & A65 & A66 & A67 & N68 & G69 & P70 & A71 & I72 \\
\hline CAG & GCG & GCG & GCA & $\mathrm{AAC}$ & GGG & $\mathrm{CCC}$ & GCC & ATC \\
\hline A73 & A74 & H75 & A76 & G77 & $\mathrm{H} 78$ & R79 & A80 & N81 \\
\hline GCA & GCC & CAT & GCC & GGA & CAC & CGG & GCC & $\mathrm{AAC}$ \\
\hline P82 & V83 & A84 & T85 & G86 & A87 & S88 & L89 & L90 \\
\hline $\mathrm{CCC}$ & GTG & GCG & $\mathrm{ACC}$ & GGT & GCT & TCG & CTG & CTG \\
\hline E91 & F92 & E93 & V94 & V95 & L96 & D97 & V98 & G99 \\
\hline GAA & TTC & GAG & GTC & GTG & CTT & GAC & GTC & GGT \\
\hline G100 & $\mathrm{T} 101$ & G102 & K103 & T104 & A105 & G106 & G107 & V108 \\
\hline GGG & $\mathrm{ACC}$ & GGC & AAG & ACT & GCA & GGA & GGT & GTA \\
\hline P109 & R110 & G111 & K112 & L113 & A114 & A115 & A116 & L117 \\
\hline $\mathrm{CCA}$ & CGA & GGT & AAG & CTT & GCG & GCC & GCA & CTC \\
\hline E118 & H119 & H120 & H121 & H122 & $\mathrm{H} 123$ & H124 & & \\
\hline GAG & CAC & CAC & CAC & CAC & CAC & CAC & & \\
\hline
\end{tabular}

Table S5. Secondary Structural Content for MhuD-heme-CN Variants

\begin{tabular}{|c|c|c|}
\hline Variant & $\alpha$ helix & $\beta$ sheet \\
\hline WT $^{\mathrm{a}}$ & 0.14 & 0.21 \\
\hline W66F $^{\mathrm{a}}$ & 0.17 & 0.19 \\
\hline W66A $^{\mathrm{a}}$ & 0.20 & 0.16 \\
\hline $\mathrm{WT}^{\mathrm{b}}$ & 0.19 & 0.31 \\
\hline
\end{tabular}

${ }^{a}$ Based upon analysis of the UV CD data presented in this work. ${ }^{b}$ Based upon X-ray crystal structure (PDB ID 4NL5). ${ }^{10}$ 
Table S6. Comparison of new and original NSD programs

\begin{tabular}{|c|c|c|c|c|c|c|c|c|c|c|}
\hline Label & $\begin{array}{c}\text { Heme } \\
\text { number }\end{array}$ & PDBID & Group & $\mathrm{D}_{\text {oop }}$ & $\operatorname{sad}\left(b_{2 u}\right)$ & $\operatorname{ruf}\left(b_{1 u}\right)$ & $\operatorname{dom}\left(a_{2 u}\right)$ & $\operatorname{wav}(\mathrm{x})\left(E_{g \mathrm{y}}\right)$ & $\operatorname{wav}(\mathrm{y})\left(E_{g \mathrm{x}}\right)$ & $\operatorname{pro}\left(a_{1 u}\right)$ \\
\hline cyt. $C_{550}$ & & $155 \mathrm{C}$ & Ref. 6 & 0.32 & 0.14 & 0.15 & 0.19 & 0.03 & 0.04 & 0.06 \\
\hline cyt. $C_{550}$ & & $155 \mathrm{C}$ & $\begin{array}{c}\text { this } \\
\text { work }\end{array}$ & 0.32 & 0.14 & 0.15 & 0.19 & 0.03 & 0.04 & 0.06 \\
\hline cyt. $C_{551}$ & & $451 \mathrm{C}$ & Ref. 6 & 0.52 & 0.09 & 0.50 & 0.03 & 0.06 & 0.00 & 0.01 \\
\hline cyt. $C_{551}$ & & $451 \mathrm{C}$ & $\begin{array}{c}\text { this } \\
\text { work }\end{array}$ & 0.52 & 0.09 & 0.50 & 0.03 & 0.06 & 0.00 & 0.01 \\
\hline cyt. $C_{553}$ & & $1 \mathrm{C} 53$ & Ref. 6 & 0.80 & 0.47 & 0.60 & 0.11 & 0.11 & 0.13 & 0.02 \\
\hline cyt. $C_{553}$ & & $1 \mathrm{C} 53$ & $\begin{array}{c}\text { this } \\
\text { work }\end{array}$ & 0.80 & 0.47 & 0.60 & 0.11 & 0.11 & 0.13 & 0.02 \\
\hline cyt. $C$ iso-2 & & 1YEA & Ref. 6 & 0.73 & 0.06 & 0.48 & 0.06 & 0.32 & 0.38 & 0.06 \\
\hline cyt. $C$ iso-2 & & 1YEA & $\begin{array}{c}\text { this } \\
\text { work }\end{array}$ & 0.73 & 0.06 & 0.48 & 0.06 & 0.32 & 0.38 & 0.06 \\
\hline cyt. $C^{\prime}$ & & $1 \mathrm{CGO}$ & Ref. 6 & 0.50 & 0.28 & 0.34 & 0.06 & 0.07 & 0.20 & 0.03 \\
\hline cyt. $C^{\prime}$ & & $1 \mathrm{CGO}$ & $\begin{array}{c}\text { this } \\
\text { work }\end{array}$ & 0.49 & 0.27 & 0.34 & 0.06 & 0.07 & 0.19 & 0.03 \\
\hline RC cyt. & 1 & 1PRC & Ref. 6 & 0.73 & 0.63 & 0.26 & 0.14 & 0.04 & 0.08 & 0.05 \\
\hline RC cyt. & 1 & 1PRC & $\begin{array}{c}\text { this } \\
\text { work }\end{array}$ & 0.73 & 0.63 & 0.26 & 0.14 & 0.04 & 0.08 & 0.05 \\
\hline RC cyt. & 2 & 1PRC & Ref. 6 & 0.57 & 0.39 & 0.38 & 0.05 & 0.04 & 0.08 & 0.04 \\
\hline RC cyt. & 2 & 1PRC & $\begin{array}{c}\text { this } \\
\text { work }\end{array}$ & 0.57 & 0.39 & 0.38 & 0.04 & 0.04 & 0.08 & 0.04 \\
\hline RC cyt. & 3 & 1PRC & Ref. 6 & 0.69 & 0.51 & 0.41 & 0.01 & 0.08 & 0.12 & 0.00 \\
\hline RC cyt. & 3 & 1PRC & $\begin{array}{c}\text { this } \\
\text { work }\end{array}$ & 0.69 & 0.51 & 0.41 & 0.01 & 0.08 & 0.12 & 0.00 \\
\hline RC cyt. & 4 & 1PRC & Ref. 6 & 0.30 & 0.17 & 0.03 & 0.11 & 0.10 & 0.16 & 0.04 \\
\hline RC cyt. & 4 & 1PRC & $\begin{array}{c}\text { this } \\
\text { work }\end{array}$ & 0.30 & 0.17 & 0.03 & 0.11 & 0.10 & 0.16 & 0.04 \\
\hline cyt. $\mathrm{P}_{450}$ & & $2 \mathrm{BMH}$ & Ref. 6 & 0.38 & 0.07 & 0.34 & 0.02 & 0.05 & 0.10 & 0.05 \\
\hline cyt. $\mathrm{P}_{450}$ & & $2 \mathrm{BMH}$ & $\begin{array}{c}\text { this } \\
\text { work }\end{array}$ & 0.38 & 0.07 & 0.33 & 0.02 & 0.05 & 0.09 & 0.05 \\
\hline bovine $b_{5}$ & & $1 \mathrm{CYO}$ & Ref. 6 & 0.52 & 0.37 & 0.31 & 0.07 & 0.11 & 0.09 & 0.03 \\
\hline bovine $b_{5}$ & & $1 \mathrm{CYO}$ & $\begin{array}{c}\text { this } \\
\text { work }\end{array}$ & 0.52 & 0.37 & 0.31 & 0.07 & 0.11 & 0.09 & 0.03 \\
\hline cyt. c. perox & & $2 \mathrm{CYP}$ & Ref. 6 & 0.73 & 0.62 & 0.24 & 0.01 & 0.18 & 0.05 & 0.01 \\
\hline cyt. $c$. perox & & $2 \mathrm{CYP}$ & $\begin{array}{c}\text { this } \\
\text { work }\end{array}$ & 0.73 & 0.62 & 0.24 & 0.01 & 0.18 & 0.05 & 0.01 \\
\hline flavocyt. $b_{2}$ & & $1 \mathrm{FCB}$ & Ref. 6 & 0.36 & 0.03 & 0.22 & 0.16 & 0.12 & 0.15 & 0.05 \\
\hline flavocyt. $b_{2}$ & & $1 \mathrm{FCB}$ & $\begin{array}{c}\text { this } \\
\text { work }\end{array}$ & 0.36 & 0.03 & 0.22 & 0.16 & 0.12 & 0.15 & 0.05 \\
\hline myoglobin & & $1 \mathrm{VXG}$ & Ref. 6 & 0.42 & 0.06 & 0.08 & 0.31 & 0.02 & 0.17 & 0.04 \\
\hline myoglobin & & $1 \mathrm{VXG}$ & $\begin{array}{c}\text { this } \\
\text { work }\end{array}$ & 0.42 & 0.06 & 0.08 & 0.31 & 0.02 & 0.17 & 0.04 \\
\hline Hildb & 1 & $2 \mathrm{CYM}$ & Ref. 6 & 0.85 & 0.06 & 0.66 & 0.32 & 0.09 & 0.23 & 0.20 \\
\hline Hildb & 1 & $2 \mathrm{CYM}$ & $\begin{array}{c}\text { this } \\
\text { work }\end{array}$ & 0.85 & 0.06 & 0.66 & 0.32 & 0.09 & 0.23 & 0.20 \\
\hline
\end{tabular}




\begin{tabular}{|c|c|c|c|c|c|c|c|c|c|c|}
\hline Hildb & 2 & $2 \mathrm{CYM}$ & Ref. 6 & 0.92 & 0.18 & 0.80 & 0.02 & 0.07 & 0.21 & 0.09 \\
\hline Hildb & 2 & $2 \mathrm{CYM}$ & $\begin{array}{c}\text { this } \\
\text { work }\end{array}$ & 0.92 & 0.18 & 0.80 & 0.02 & 0.07 & 0.21 & 0.09 \\
\hline Hildb & 3 & $2 \mathrm{CYM}$ & Ref. 6 & 0.67 & 0.32 & 0.27 & 0.06 & 0.03 & 0.01 & 0.22 \\
\hline Hildb & 3 & $2 \mathrm{CYM}$ & $\begin{array}{c}\text { this } \\
\text { work } \\
\end{array}$ & 0.67 & 0.32 & 0.27 & 0.06 & 0.03 & 0.01 & 0.22 \\
\hline Hildb & 4 & $2 \mathrm{CYM}$ & Ref. 6 & 1.24 & 1.02 & 0.35 & 0.27 & 0.24 & 0.33 & 0.15 \\
\hline Hildb & 4 & $2 \mathrm{CYM}$ & $\begin{array}{c}\text { this } \\
\text { work }\end{array}$ & 1.24 & 1.02 & 0.35 & 0.27 & 0.32 & 0.24 & 0.15 \\
\hline Miyaz & 1 & $2 \mathrm{CDV}$ & Ref. 6 & 0.89 & 0.17 & 0.84 & 0.14 & 0.07 & 0.02 & 0.01 \\
\hline Miyaz & 1 & $2 \mathrm{CDV}$ & $\begin{array}{c}\text { this } \\
\text { work }\end{array}$ & 0.89 & 0.17 & 0.84 & 0.14 & 0.07 & 0.02 & 0.01 \\
\hline Miyaz & 2 & $2 \mathrm{CDV}$ & Ref. 6 & 0.94 & 0.16 & 0.88 & 0.07 & 0.22 & 0.07 & 0.04 \\
\hline Miyaz & 2 & $2 \mathrm{CDV}$ & $\begin{array}{c}\text { this } \\
\text { work }\end{array}$ & 0.94 & 0.16 & 0.88 & 0.07 & 0.22 & 0.07 & 0.04 \\
\hline Miyaz & 3 & $2 \mathrm{CDV}$ & Ref. 6 & 0.56 & 0.37 & 0.39 & 0.01 & 0.09 & 0.02 & 0.03 \\
\hline Miyaz & 3 & $2 \mathrm{CDV}$ & $\begin{array}{c}\text { this } \\
\text { work }\end{array}$ & 0.56 & 0.37 & 0.39 & 0.01 & 0.09 & 0.02 & 0.03 \\
\hline Miyaz & 4 & $2 \mathrm{CDV}$ & Ref. 6 & 1.18 & 1.03 & 0.43 & 0.06 & 0.11 & 0.21 & 0.03 \\
\hline Miyaz & 4 & $2 \mathrm{CDV}$ & $\begin{array}{c}\text { this } \\
\text { work }\end{array}$ & 1.18 & 1.03 & 0.43 & 0.06 & 0.11 & 0.21 & 0.03 \\
\hline Norw. & 1 & $2 \mathrm{CY} 3$ & Ref. 6 & 0.79 & 0.27 & 0.69 & 0.12 & 0.06 & 0.08 & 0.03 \\
\hline Norw. & 1 & $2 \mathrm{CY} 3$ & $\begin{array}{c}\text { this } \\
\text { work }\end{array}$ & 0.79 & 0.27 & 0.69 & 0.12 & 0.06 & 0.08 & 0.03 \\
\hline Norw. & 2 & $2 \mathrm{CY} 3$ & Ref. 6 & 1.27 & 0.48 & 1.14 & 0.12 & 0.15 & 0.06 & 0.09 \\
\hline Norw. & 2 & $2 \mathrm{CY} 3$ & $\begin{array}{c}\text { this } \\
\text { work }\end{array}$ & 1.27 & 0.48 & 1.14 & 0.12 & 0.15 & 0.06 & 0.09 \\
\hline Norw. & 3 & $2 \mathrm{CY} 3$ & Ref. 6 & 0.33 & 0.07 & 0.27 & 0.01 & 0.09 & 0.05 & 0.00 \\
\hline Norw. & 3 & $2 \mathrm{CY} 3$ & $\begin{array}{c}\text { this } \\
\text { work }\end{array}$ & 0.33 & 0.07 & 0.27 & 0.01 & 0.08 & 0.05 & 0.00 \\
\hline Norw. & 4 & $2 \mathrm{CY} 3$ & Ref. 6 & 0.80 & 0.16 & 0.69 & 0.06 & 0.09 & 0.31 & 0.03 \\
\hline Norw. & 4 & $2 \mathrm{CY} 3$ & $\begin{array}{c}\text { this } \\
\text { work }\end{array}$ & 0.80 & 0.16 & 0.69 & 0.05 & 0.09 & 0.31 & 0.02 \\
\hline $\begin{array}{c}\text { ferrocyt. } c \\
W T\end{array}$ & & $1 Y C C$ & Ref. 6 & 0.89 & 0.31 & 0.77 & 0.01 & 0.14 & 0.20 & 0.10 \\
\hline $\begin{array}{c}\text { ferrocyt. } c \\
W T\end{array}$ & & $1 Y C C$ & $\begin{array}{c}\text { this } \\
\text { work }\end{array}$ & 0.89 & 0.31 & 0.77 & 0.01 & 0.14 & 0.20 & 0.10 \\
\hline $\begin{array}{c}\text { ferrocyt. } c \\
\text { N52I }\end{array}$ & & 1CRH & Ref. 6 & 1.12 & 0.20 & 1.02 & 0.02 & 0.33 & 0.16 & 0.00 \\
\hline $\begin{array}{c}\text { ferrocyt. } c \\
\text { N52I }\end{array}$ & & 1CRH & $\begin{array}{c}\text { this } \\
\text { work }\end{array}$ & 1.12 & 0.20 & 1.02 & 0.02 & 0.33 & 0.16 & 0.00 \\
\hline $\begin{array}{c}\text { ferrocyt. } c \\
\text { N52I C102T }\end{array}$ & & 1CRG & Ref. 6 & 1.00 & 0.30 & 0.90 & 0.08 & 0.13 & 0.18 & 0.04 \\
\hline $\begin{array}{c}\text { ferrocyt. } c \\
\text { N52I C102T }\end{array}$ & & $1 \mathrm{CRG}$ & $\begin{array}{c}\text { this } \\
\text { work }\end{array}$ & 1.00 & 0.30 & 0.90 & 0.08 & 0.13 & 0.18 & 0.04 \\
\hline $\begin{array}{c}\text { ferrocyt. } c \\
\text { Y67F C102T }\end{array}$ & & $1 \mathrm{CTZ}$ & Ref. 6 & 1.04 & 0.10 & 0.95 & 0.01 & 0.19 & 0.33 & 0.02 \\
\hline $\begin{array}{c}\text { ferrocyt. } c \\
\text { Y67F C102T }\end{array}$ & & $1 \mathrm{CTZ}$ & $\begin{array}{c}\text { this } \\
\text { work }\end{array}$ & 1.04 & 0.10 & 0.95 & 0.01 & 0.19 & 0.33 & 0.02 \\
\hline $\begin{array}{c}\text { ferrocyt. } c \\
\text { F82Y C102T }\end{array}$ & & $1 \mathrm{CHH}$ & Ref. 6 & 0.91 & 0.05 & 0.86 & 0.04 & 0.22 & 0.13 & 0.01 \\
\hline
\end{tabular}




\begin{tabular}{|c|c|c|c|c|c|c|c|c|c|}
\hline $\begin{array}{c}\text { ferrocyt. } c \\
\text { F82Y C102T }\end{array}$ & $1 \mathrm{CHH}$ & $\begin{array}{c}\text { this } \\
\text { work }\end{array}$ & 0.91 & 0.05 & 0.86 & 0.04 & 0.21 & 0.13 & 0.01 \\
\hline $\begin{array}{l}\text { ferrocyt. } c \\
\text { L85M C102T }\end{array}$ & 1CSW & Ref. 6 & 0.97 & 0.30 & 0.86 & 0.11 & 0.04 & 0.22 & 0.01 \\
\hline $\begin{array}{l}\text { ferrocyt. } c \\
\text { L85M C102T }\end{array}$ & $1 \mathrm{CSW}$ & $\begin{array}{c}\text { this } \\
\text { work }\end{array}$ & 0.97 & 0.30 & 0.86 & 0.11 & 0.04 & 0.22 & 0.01 \\
\hline $\begin{array}{c}\text { ferrocyt. } c \\
\text { L85A C102T }\end{array}$ & $1 \mathrm{CHJ}$ & Ref. 6 & 0.84 & 0.00 & 0.77 & 0.03 & 0.20 & 0.21 & 0.04 \\
\hline $\begin{array}{c}\text { ferrocyt. } c \\
\text { L85A C } 102 \mathrm{~T}\end{array}$ & $1 \mathrm{CHJ}$ & $\begin{array}{c}\text { this } \\
\text { work } \\
\end{array}$ & 0.84 & 0.00 & 0.77 & 0.03 & 0.20 & 0.21 & 0.04 \\
\hline $\begin{array}{c}\text { ferrocyt. } c \\
\text { L85C C102T }\end{array}$ & $1 \mathrm{CSU}$ & Ref. 6 & 0.92 & 0.23 & 0.83 & 0.09 & 0.07 & 0.23 & 0.00 \\
\hline $\begin{array}{c}\text { ferrocyt. } c \\
\text { L85C C102T }\end{array}$ & $1 \mathrm{CSU}$ & $\begin{array}{c}\text { this } \\
\text { work }\end{array}$ & 0.92 & 0.23 & 0.83 & 0.09 & 0.07 & 0.23 & 0.00 \\
\hline $\begin{array}{c}\text { ferrocyt. } c \\
\text { L85F C102T }\end{array}$ & $1 \mathrm{CSV}$ & Ref. 6 & 0.89 & 0.01 & 0.76 & 0.18 & 0.14 & 0.36 & 0.01 \\
\hline $\begin{array}{c}\text { ferrocyt. } c \\
\text { L85F C102T }\end{array}$ & 1CSV & $\begin{array}{c}\text { this } \\
\text { work }\end{array}$ & 0.89 & 0.01 & 0.76 & 0.18 & 0.14 & 0.36 & 0.01 \\
\hline $\begin{array}{c}\text { ferrocyt. } c \\
\text { L94S C102T }\end{array}$ & $1 \mathrm{CSX}$ & Ref. 6 & 0.99 & 0.14 & 0.85 & 0.01 & 0.29 & 0.33 & 0.03 \\
\hline $\begin{array}{c}\text { ferrocyt. } c \\
\text { L94S C102T }\end{array}$ & 1CSX & $\begin{array}{c}\text { this } \\
\text { work }\end{array}$ & 0.99 & 0.14 & 0.84 & 0.01 & 0.29 & 0.33 & 0.03 \\
\hline $\begin{array}{c}\text { ferrocyt. } c \\
\text { R38A F82S } \\
\text { C102T }\end{array}$ & $1 \mathrm{CIF}$ & Ref. 6 & 1.23 & 0.37 & 1.10 & 0.08 & 0.09 & 0.30 & 0.03 \\
\hline $\begin{array}{c}\text { ferrocyt. } c \\
\text { R38A F82S } \\
\text { C102T }\end{array}$ & $1 \mathrm{CIF}$ & $\begin{array}{c}\text { this } \\
\text { work }\end{array}$ & 1.23 & 0.37 & 1.10 & 0.08 & 0.09 & 0.30 & 0.03 \\
\hline $\begin{array}{c}\text { ferrocyt. } c \\
\text { N52I F82S } \\
\text { C102A }\end{array}$ & 1CIE & Ref. 6 & 0.81 & 0.09 & 0.69 & 0.03 & 0.22 & 0.30 & 0.01 \\
\hline $\begin{array}{c}\text { ferrocyt. } c \\
\text { N52I F82S } \\
\text { C102A }\end{array}$ & 1CIE & $\begin{array}{c}\text { this } \\
\text { work }\end{array}$ & 0.81 & 0.09 & 0.69 & 0.03 & 0.22 & 0.30 & 0.01 \\
\hline $\begin{array}{c}\text { ferrocyt. } c \\
\text { N52I y67F } \\
\text { C102T }\end{array}$ & 1CRJ & Ref. 6 & 0.79 & 0.16 & 0.69 & 0.06 & 0.14 & 0.24 & 0.00 \\
\hline $\begin{array}{c}\text { ferrocyt. } c \\
\text { N52I y67F } \\
\text { C102T }\end{array}$ & 1CRJ & $\begin{array}{c}\text { this } \\
\text { work }\end{array}$ & 0.79 & 0.16 & 0.69 & 0.06 & 0.14 & 0.24 & 0.00 \\
\hline $\begin{array}{c}\text { ferrocyt. } c \\
\text { F82Y L85A } \\
\text { C102T } \\
\end{array}$ & $1 \mathrm{CHI}$ & Ref. 6 & 0.92 & 0.07 & 0.80 & 0.04 & 0.27 & 0.33 & 0.01 \\
\hline $\begin{array}{c}\text { ferrocyt. } c \\
\text { F82Y L85A } \\
\text { C102T }\end{array}$ & $1 \mathrm{CHI}$ & $\begin{array}{l}\text { this } \\
\text { work }\end{array}$ & 0.92 & 0.07 & 0.80 & 0.04 & 0.24 & 0.33 & 0.01 \\
\hline $\begin{array}{c}\text { ferrocyt. } c \\
\text { E21D K22Q } \\
\text { P25A H26N }\end{array}$ & $1 \mathrm{RAQ}$ & Ref. 6 & 0.90 & 0.18 & 0.81 & 0.08 & 0.12 & 0.26 & 0.03 \\
\hline $\begin{array}{c}\text { ferrocyt. } c \\
\text { E21D K22Q } \\
\text { P25A H26N }\end{array}$ & $1 \mathrm{RAQ}$ & $\begin{array}{l}\text { this } \\
\text { work }\end{array}$ & 0.90 & 0.18 & 0.81 & 0.08 & 0.12 & 0.26 & 0.03 \\
\hline $\begin{array}{c}\text { ferrocyt. } c \\
\text { E21D K22Q } \\
\text { P25A H26N }\end{array}$ & 1RAP & Ref. 6 & 0.80 & 0.24 & 0.68 & 0.04 & 0.23 & 0.19 & 0.01 \\
\hline
\end{tabular}




\begin{tabular}{|c|c|c|c|c|c|c|c|c|c|}
\hline $\begin{array}{c}\text { ferrocyt. } c \\
\text { E21D K22Q } \\
\text { P25A H26N }\end{array}$ & 1RAP & $\begin{array}{c}\text { this } \\
\text { work }\end{array}$ & 0.80 & 0.24 & 0.68 & 0.04 & 0.23 & 0.18 & 0.01 \\
\hline
\end{tabular}

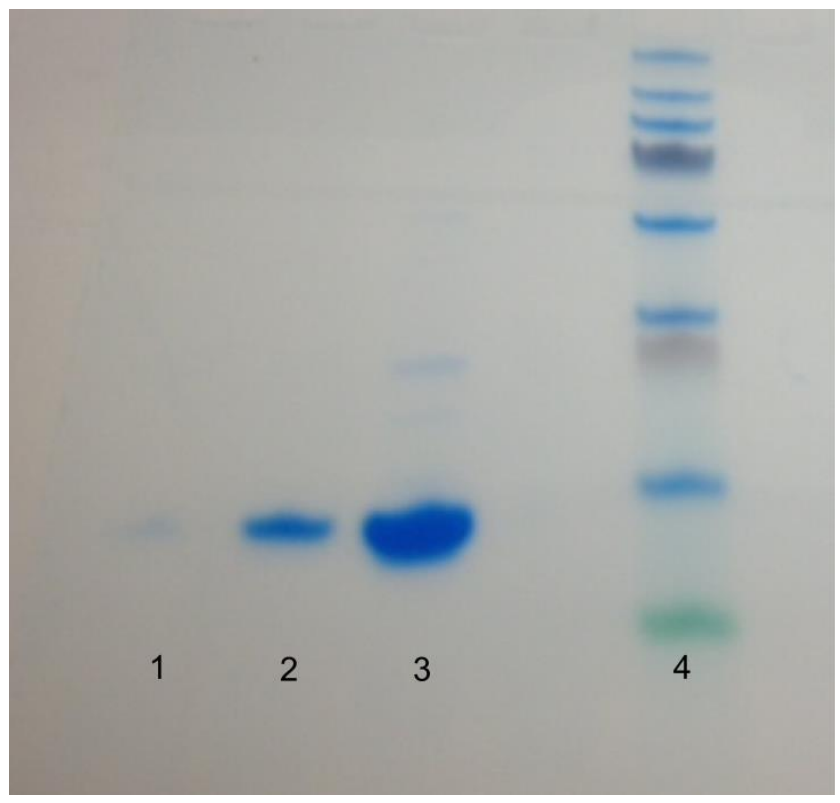

Figure S1. SDS-PAGE gel of W66F MhuD. 1/1000 dilution of W66F MhuD (1), 1/10dilution of W66F MhuD (2), >95\% Pure W66F MhuD (3), and molecular weight ladder (4). 


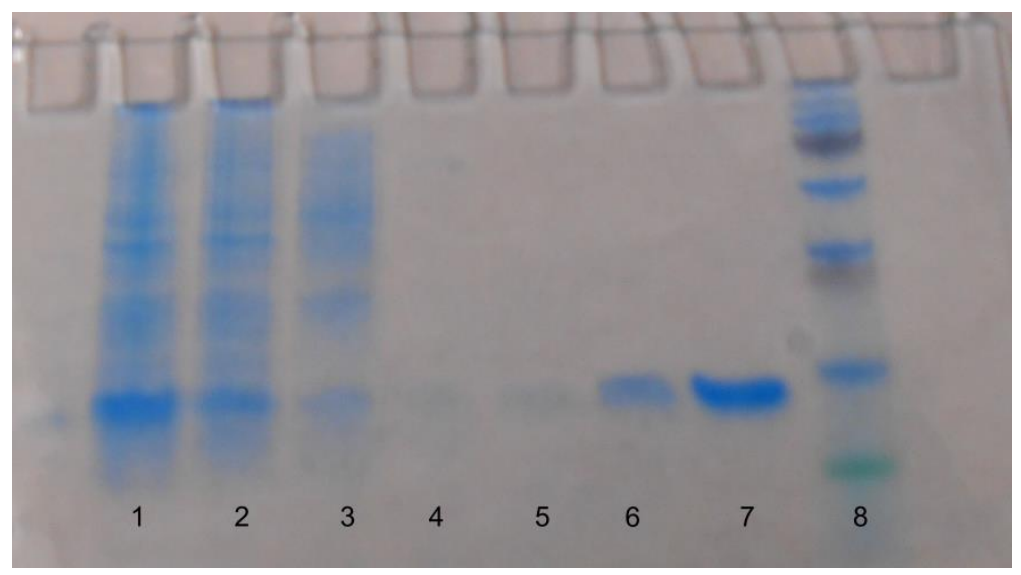

Figure S2. SDS-PAGE gel of W66A MhuD. Lysate (1), flow through (2), $20 \mathrm{mM}$ Tris, pH 7.8, $50 \mathrm{mM} \mathrm{NaCl}, 20 \mathrm{mM}$ imidazole wash (3), $20 \mathrm{mM}$ Tris, $\mathrm{pH} 7.8,50 \mathrm{mM} \mathrm{NaCl}, 50 \mathrm{mM}$ imidazole wash (4), 1/100 dilution of W66A MhuD (5), 1/10 dilution of W66A MhuD (6), Pure W66A MhuD (7), and molecular weight ladder (8).

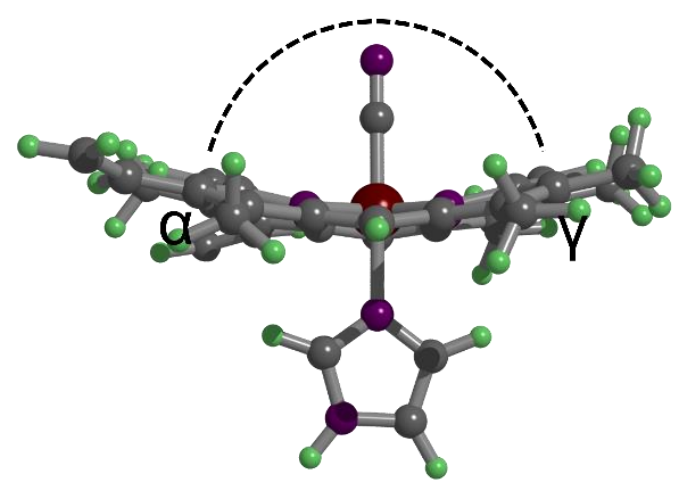

Figure S3. $\alpha$-meso-Fe- $\gamma$-meso angle constraint. 


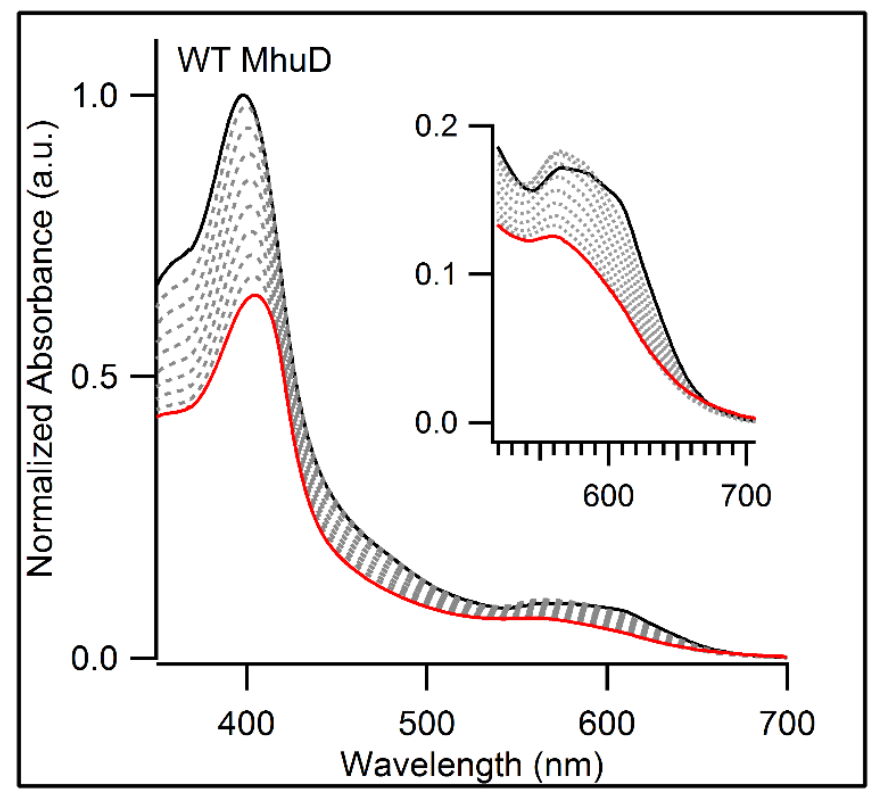

Figure S4 Heme degradation by WT MhuD with the initial (black), intermediate (grey), and final (red) UV/Vis Abs spectra shown. $1 \mathrm{mM}$ protein-heme was combined with $10 \mathrm{mM}$ ascorbate and $5 \mu \mathrm{M}$ catalase in $20 \mathrm{mM}$ Tris, $\mathrm{pH} 7.4,50 \mathrm{mM} \mathrm{NaCl}$.

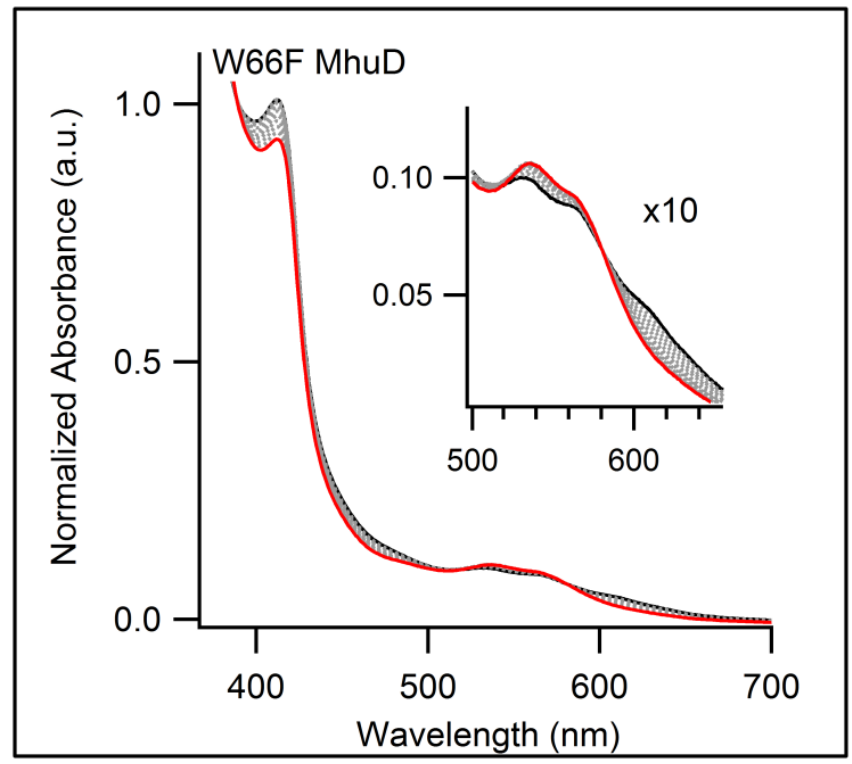

Figure S5. Heme degradation by W66F Mhu with the initial (black), intermediate (grey), and final (red) UV/Vis Abs spectra shown. $1 \mathrm{mM}$ protein-heme was combined with $10 \mathrm{mM}$ ascorbate and $5 \mu \mathrm{M}$ catalase in $20 \mathrm{mM}$ Tris, $\mathrm{pH} 7.4,50 \mathrm{mM} \mathrm{NaCl}$.. 


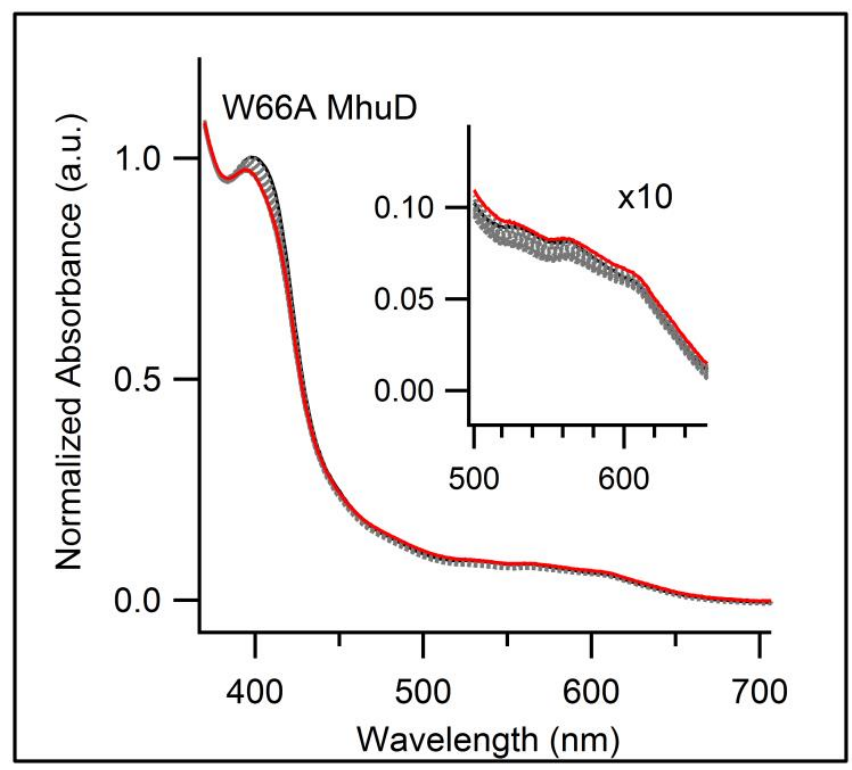

Figure S6 Heme degradation by W66A MhuD with the initial (black), intermediate (grey), and final (red) UV/Vis Abs spectra shown. $1 \mathrm{mM}$ protein-heme was combined with $10 \mathrm{mM}$ ascorbate and $5 \mu \mathrm{M}$ catalase in $20 \mathrm{mM}$ Tris, $\mathrm{pH} 7.4,50 \mathrm{mM} \mathrm{NaCl}$.

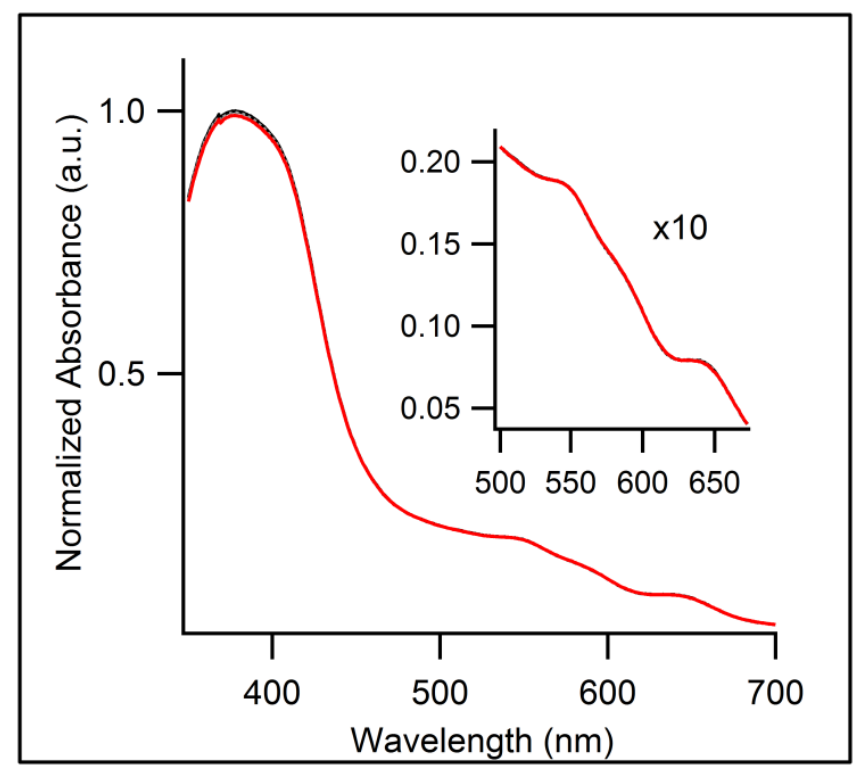

Figure S7. Initial (black), intermediate (grey), and final (red) UV/Vis Abs spectra shown.1 $\mathrm{mM}$ heme was combined with $10 \mathrm{mM}$ ascorbate and $5 \mu \mathrm{M}$ catalase in $20 \mathrm{mM}$ Tris, $\mathrm{pH} 7.4,50 \mathrm{mM} \mathrm{NaCl}$. 


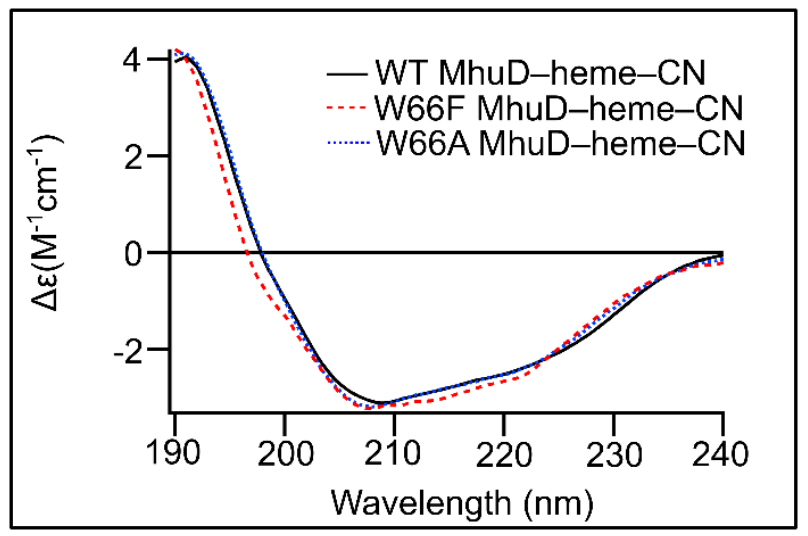

Figure S8. UV CD spectra of WT MhuD-heme-CN (black, solid line), W66F MhuD-heme-CN (red, dashed line), and W66A MhuD-heme-CN (blue, dotted line) in $10 \mu \mathrm{M}$ potassium phosphate buffer, $\mathrm{pH}$ 7.4 .

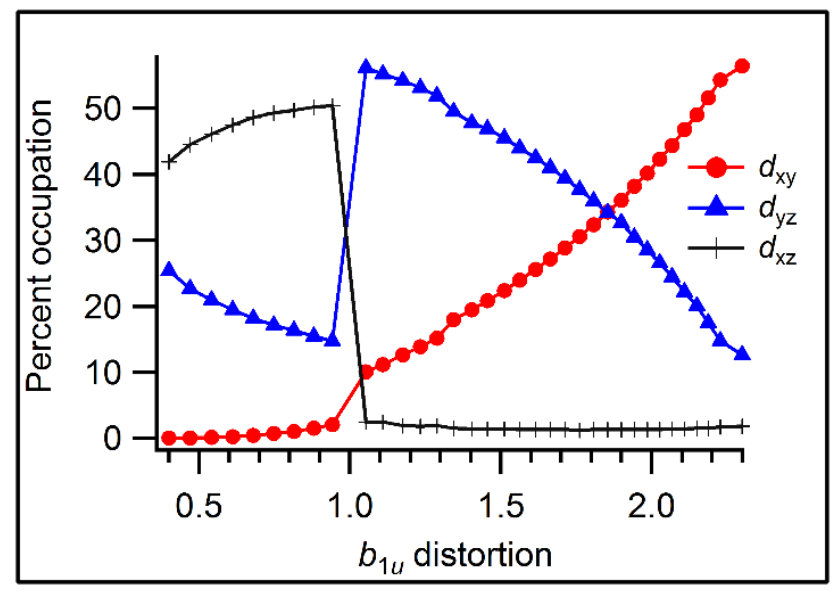

Figure S9. The individual $d$ orbital contributions to the singly occupied molecular orbital by Löwdin analysis for each geometry. 


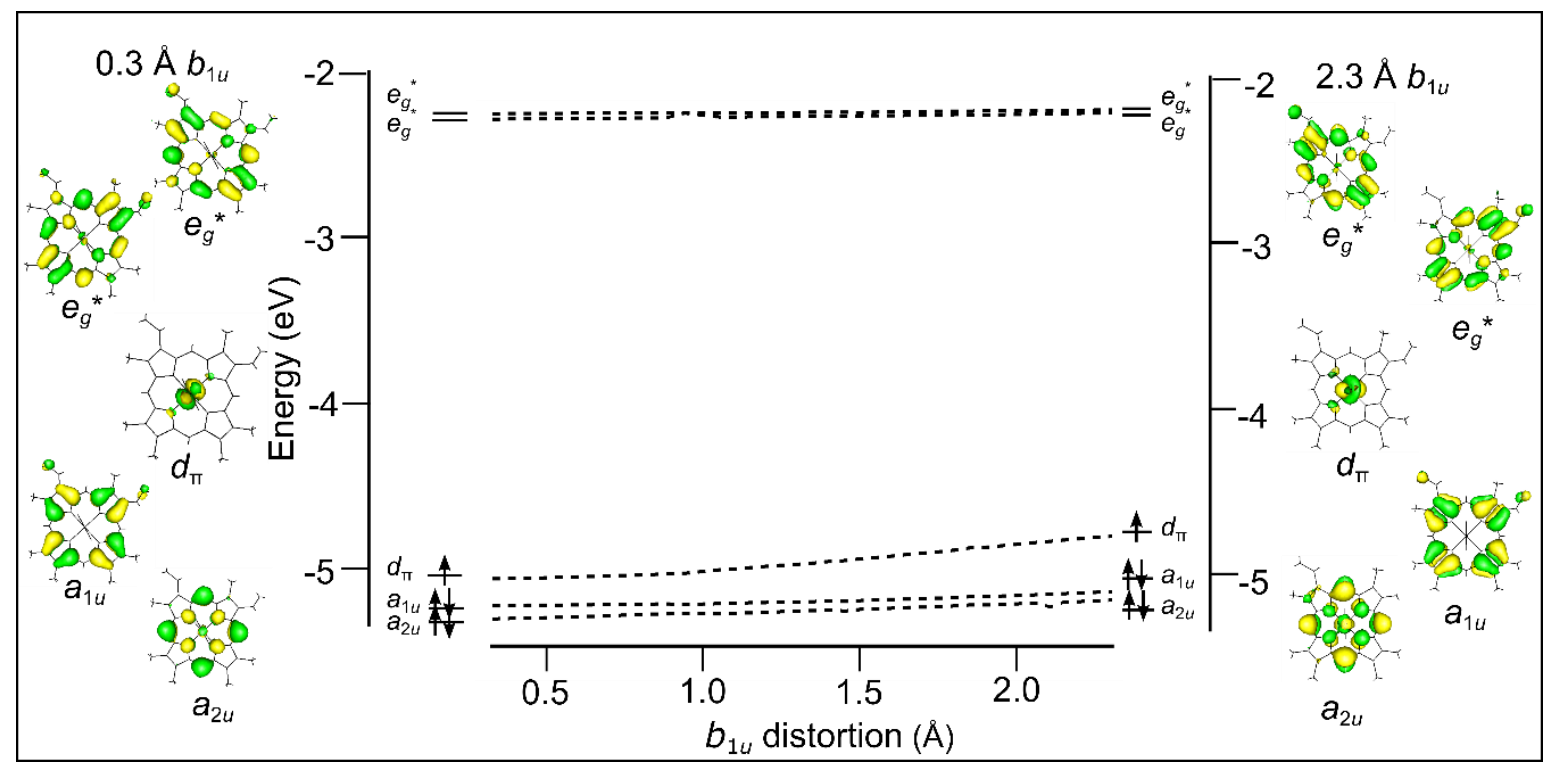

Figure S10. PBE0/TZVP-computed valence QROs for cyanide, imidazole-ligated ferric heme as a function of porphyrin ruffling. Valence QRO plots for models with $0.3 \AA$ (left) and $2.3 \AA$ (right) ruffling are shown, in addition to the influence of ruffling on the energy of each QRO (middle).

\section{Cartesian Coordinates for all Computational Models}

His-heme-CN, $161.5^{\circ} \alpha$-meso-Fe- $\gamma$-meso angle

$\begin{array}{lrrr}\mathrm{C} & 0.508110 & -0.343336 & -4.196620 \\ \mathrm{~N} & 1.324282 & 0.731600 & -3.892087 \\ \mathrm{C} & -0.041687 & -0.735263 & -3.001339 \\ \mathrm{C} & 1.248850 & 0.956353 & -2.553662 \\ \mathrm{~N} & 0.426347 & 0.080753 & -1.991173 \\ \mathrm{H} & 0.398487 & -0.719814 & -5.206741 \\ \mathrm{H} & 1.885424 & 1.262585 & -4.549844 \\ \mathrm{H} & -0.733627 & -1.543586 & -2.793447 \\ \mathrm{H} & 1.787940 & 1.740561 & -2.033085 \\ \mathrm{C} & -2.444587 & -2.412618 & -0.038818 \\ \mathrm{C} & -2.293493 & 2.373334 & -0.745122 \\ \mathrm{C} & 2.136338 & 2.325922 & 1.193383 \\ \mathrm{C} & 2.381363 & -2.384457 & 0.114540 \\ \mathrm{C} & -2.832087 & -1.101888 & -0.285136 \\ \mathrm{C} & -4.183201 & -0.685790 & -0.601493 \\ \mathrm{C} & -4.119552 & 0.655768 & -0.903093 \\ \mathrm{C} & -2.736845 & 1.058496 & -0.719791 \\ \mathrm{C} & -5.230822 & 1.578136 & -1.288918 \\ \mathrm{C} & -5.375940 & -1.587127 & -0.591485 \\ \mathrm{C} & -1.056482 & 2.805562 & -0.270657 \\ \mathrm{C} & -0.722315 & 4.186178 & 0.003240 \\ \mathrm{C} & 0.499570 & 4.175065 & 0.657932 \\ \mathrm{C} & 0.914031 & 2.777295 & 0.715962 \\ \mathrm{C} & -1.564900 & 5.361050 & -0.368546 \\ & & & \end{array}$




\begin{tabular}{|c|c|c|c|}
\hline C & 1.291133 & 5.279747 & 1.168146 \\
\hline $\mathrm{C}$ & 0.872790 & 6.522458 & 1.481834 \\
\hline $\mathrm{C}$ & 2.631776 & 1.033403 & 1.022996 \\
\hline $\mathrm{C}$ & 3.988911 & 0.628005 & 1.300892 \\
\hline $\mathrm{C}$ & 4.096031 & -0.692288 & 0.883390 \\
\hline $\mathrm{C}$ & 2.766283 & -1.080889 & 0.427319 \\
\hline $\mathrm{C}$ & 5.028125 & 1.482859 & 1.948403 \\
\hline $\mathrm{C}$ & 5.241350 & -1.583467 & 0.922259 \\
\hline $\mathrm{C}$ & 6.545326 & -1.242085 & 0.950140 \\
\hline $\mathrm{C}$ & 1.072171 & -2.823463 & -0.030738 \\
\hline $\mathrm{C}$ & 0.672249 & -4.224954 & -0.032363 \\
\hline $\mathrm{C}$ & -0.699971 & -4.237124 & 0.025904 \\
\hline C & -1.122664 & -2.845085 & 0.008467 \\
\hline $\mathrm{C}$ & 1.620076 & -5.380243 & -0.038115 \\
\hline $\mathrm{C}$ & -1.628855 & -5.406514 & 0.086663 \\
\hline $\mathrm{N}$ & -1.964360 & -0.028067 & -0.369162 \\
\hline $\mathrm{N}$ & -0.045349 & 1.967633 & 0.142693 \\
\hline $\mathrm{N}$ & 1.914499 & -0.009200 & 0.479763 \\
\hline $\mathrm{N}$ & -0.035617 & -2.010013 & -0.044091 \\
\hline $\mathrm{Fe}$ & -0.044671 & -0.013950 & 0.042873 \\
\hline $\mathrm{H}$ & -3.227635 & -3.169443 & 0.018521 \\
\hline $\mathrm{H}$ & -3.018373 & 3.136426 & -1.028287 \\
\hline $\mathrm{H}$ & 2.803471 & 3.057658 & 1.646961 \\
\hline $\mathrm{H}$ & 3.157358 & -3.149895 & 0.110760 \\
\hline $\mathrm{H}$ & -6.149419 & 1.023139 & -1.520336 \\
\hline $\mathrm{H}$ & -4.972738 & 2.177588 & -2.176291 \\
\hline $\mathrm{H}$ & -5.469581 & 2.286741 & -0.478580 \\
\hline $\mathrm{H}$ & -5.573980 & -1.981508 & 0.417863 \\
\hline $\mathrm{H}$ & -5.238526 & -2.454117 & -1.257051 \\
\hline $\mathrm{H}$ & -6.280509 & -1.058702 & -0.920393 \\
\hline $\mathrm{H}$ & -2.204588 & 5.690262 & 0.468163 \\
\hline $\mathrm{H}$ & -2.226941 & 5.133758 & -1.215933 \\
\hline $\mathrm{H}$ & -0.934911 & 6.218861 & -0.648850 \\
\hline $\mathrm{H}$ & 2.353966 & 5.064275 & 1.322877 \\
\hline $\mathrm{H}$ & -0.169848 & 6.830987 & 1.413485 \\
\hline $\mathrm{H}$ & 1.579452 & 7.266436 & 1.851728 \\
\hline $\mathrm{H}$ & 4.575897 & 2.253748 & 2.588167 \\
\hline $\mathrm{H}$ & 5.665785 & 1.999808 & 1.210813 \\
\hline $\mathrm{H}$ & 5.694236 & 0.871887 & 2.575805 \\
\hline $\mathrm{H}$ & 5.009003 & -2.654066 & 0.921838 \\
\hline $\mathrm{H}$ & 6.884735 & -0.207079 & 0.908776 \\
\hline $\mathrm{H}$ & 7.319123 & -2.009550 & 0.995065 \\
\hline $\mathrm{H}$ & 2.202671 & -5.431986 & 0.896658 \\
\hline $\mathrm{H}$ & 2.342512 & -5.312783 & -0.867479 \\
\hline $\mathrm{H}$ & 1.086698 & -6.334240 & -0.142328 \\
\hline $\mathrm{H}$ & -1.080438 & -6.356377 & 0.037607 \\
\hline $\mathrm{H}$ & -2.351601 & -5.400493 & -0.745938 \\
\hline $\mathrm{H}$ & -2.212357 & -5.411185 & 1.021573 \\
\hline $\mathrm{C}$ & -0.367586 & -0.186306 & 1.900166 \\
\hline $\mathrm{N}$ & -0.580346 & -0.286359 & 3.050616 \\
\hline
\end{tabular}

His-heme-CN, $162.5^{\circ} \alpha$-meso-Fe- $\gamma$-meso angle 


\begin{tabular}{|c|c|c|c|}
\hline $\mathrm{C}$ & 0.507851 & -0.343690 & -4.189847 \\
\hline $\mathrm{N}$ & 1.320010 & 0.734440 & -3.885836 \\
\hline $\mathrm{C}$ & -0.040536 & -0.737028 & -2.994368 \\
\hline $\mathrm{C}$ & 1.243608 & 0.959612 & -2.547528 \\
\hline $\mathrm{N}$ & 0.424362 & 0.081276 & -1.984618 \\
\hline $\mathrm{H}$ & 0.399667 & -0.721113 & -5.199775 \\
\hline $\mathrm{H}$ & 1.879241 & 1.267188 & -4.543800 \\
\hline $\mathrm{H}$ & -0.729395 & -1.547906 & -2.786224 \\
\hline $\mathrm{H}$ & 1.779734 & 1.746209 & -2.027464 \\
\hline $\mathrm{C}$ & -2.445851 & -2.418237 & -0.059326 \\
\hline $\mathrm{C}$ & -2.300520 & 2.373994 & -0.724494 \\
\hline $\mathrm{C}$ & 2.145609 & 2.332467 & 1.177538 \\
\hline C & 2.379100 & -2.387021 & 0.134700 \\
\hline $\mathrm{C}$ & -2.833507 & -1.105698 & -0.294420 \\
\hline $\mathrm{C}$ & -4.184665 & -0.689253 & -0.609864 \\
\hline $\mathrm{C}$ & -4.123144 & 0.655601 & -0.896062 \\
\hline C & -2.741521 & 1.058558 & -0.705709 \\
\hline C & -5.235254 & 1.579848 & -1.274961 \\
\hline C & -5.375381 & -1.593272 & -0.612520 \\
\hline C & -1.061738 & 2.806070 & -0.255485 \\
\hline $\mathrm{C}$ & -0.725786 & 4.187384 & 0.011856 \\
\hline $\mathrm{C}$ & 0.503375 & 4.178417 & 0.652645 \\
\hline $\mathrm{C}$ & 0.918479 & 2.780944 & 0.710323 \\
\hline $\mathrm{C}$ & -1.572352 & 5.361310 & -0.353875 \\
\hline C & 1.300504 & 5.284599 & 1.150768 \\
\hline C & 0.886164 & 6.528957 & 1.463350 \\
\hline C & 2.636356 & 1.037395 & 1.016611 \\
\hline C & 3.993279 & 0.631801 & 1.294862 \\
\hline C & 4.095734 & -0.693237 & 0.891953 \\
\hline C & 2.764303 & -1.082651 & 0.442064 \\
\hline C & 5.036266 & 1.491069 & 1.930356 \\
\hline C & 5.238677 & -1.587083 & 0.938286 \\
\hline $\mathrm{C}$ & 6.543610 & -1.248914 & 0.959033 \\
\hline $\mathrm{C}$ & 1.070568 & -2.825047 & -0.016796 \\
\hline $\mathrm{C}$ & 0.671925 & -4.226677 & -0.025955 \\
\hline $\mathrm{C}$ & -0.700792 & -4.240014 & 0.016447 \\
\hline $\mathrm{C}$ & -1.123967 & -2.848327 & -0.001059 \\
\hline $\mathrm{C}$ & 1.620619 & -5.381248 & -0.025391 \\
\hline $\mathrm{C}$ & -1.629511 & -5.410139 & 0.063681 \\
\hline $\mathrm{N}$ & -1.967171 & -0.029564 & -0.363993 \\
\hline $\mathrm{N}$ & -0.046657 & 1.968653 & 0.150110 \\
\hline $\mathrm{N}$ & 1.914169 & -0.008972 & 0.486337 \\
\hline $\mathrm{N}$ & -0.037123 & -2.011469 & -0.038624 \\
\hline $\mathrm{Fe}$ & -0.046810 & -0.014047 & 0.051682 \\
\hline $\mathrm{H}$ & -3.228067 & -3.176666 & -0.014168 \\
\hline $\mathrm{H}$ & -3.027203 & 3.137119 & -1.002775 \\
\hline $\mathrm{H}$ & 2.817545 & 3.066552 & 1.619931 \\
\hline $\mathrm{H}$ & 3.154443 & -3.153067 & 0.135477 \\
\hline $\mathrm{H}$ & -6.152339 & 1.025493 & -1.513872 \\
\hline $\mathrm{H}$ & -4.976435 & 2.188643 & -2.155708 \\
\hline $\mathrm{H}$ & -5.476965 & 2.279879 & -0.458073 \\
\hline $\mathrm{H}$ & -5.573975 & -2.000107 & 0.391790 \\
\hline
\end{tabular}




\begin{tabular}{|c|c|c|c|}
\hline $\mathrm{H}$ & & & \\
\hline & -5.235277 & -2.451998 & -1.288208 \\
\hline $\mathrm{H}$ & -6.280608 & -1.062749 & -0.936249 \\
\hline $\mathrm{H}$ & -2.199493 & 5.695524 & 0.490351 \\
\hline $\mathrm{H}$ & -2.246748 & 5.130658 & -1.190436 \\
\hline $\mathrm{H}$ & -0.945645 & 6.217074 & -0.647687 \\
\hline $\mathrm{H}$ & 2.364661 & 5.069125 & 1.296153 \\
\hline $\mathrm{H}$ & -0.156757 & 6.838206 & 1.403534 \\
\hline $\mathrm{H}$ & 1.596934 & 7.273751 & 1.823599 \\
\hline $\mathrm{H}$ & 4.587552 & 2.270886 & 2.561685 \\
\hline $\mathrm{H}$ & 5.674189 & 1.997345 & 1.185649 \\
\hline $\mathrm{H}$ & 5.701581 & 0.885508 & 2.563879 \\
\hline $\mathrm{H}$ & 5.003480 & -2.656950 & 0.951332 \\
\hline $\mathrm{H}$ & 6.885753 & -0.215428 & 0.904422 \\
\hline $\mathrm{H}$ & 7.315411 & -2.017937 & 1.011222 \\
\hline $\mathrm{H}$ & 2.194704 & -5.434366 & 0.914503 \\
\hline $\mathrm{H}$ & 2.350509 & -5.311550 & -0.848033 \\
\hline $\mathrm{H}$ & 1.088902 & -6.335408 & -0.136449 \\
\hline $\mathrm{H}$ & -1.079834 & -6.359458 & 0.018706 \\
\hline $\mathrm{H}$ & -2.342722 & -5.402824 & -0.777074 \\
\hline $\mathrm{H}$ & -2.223587 & -5.417481 & 0.991927 \\
\hline C & -0.369447 & -0.187560 & 1.908382 \\
\hline $\mathrm{N}$ & -0.582125 & -0.288371 & 3.058824 \\
\hline His $-\mathrm{r}$ & $\mathrm{N}, 163.4^{\circ} \alpha-\mathrm{me}$ & e- $\gamma$-meso angle & \\
\hline $\mathrm{C}$ & 0.511281 & -0.343618 & -4.182876 \\
\hline $\mathrm{N}$ & 1.315492 & 0.740494 & -3.878972 \\
\hline C & -0.035382 & -0.739933 & -2.987585 \\
\hline $\mathrm{C}$ & 1.236462 & 0.965986 & -2.540877 \\
\hline $\mathrm{N}$ & 0.423009 & 0.082253 & -1.978032 \\
\hline $\mathrm{H}$ & 0.406602 & -0.722478 & -5.192642 \\
\hline $\mathrm{H}$ & 1.871403 & 1.276793 & -4.536869 \\
\hline $\mathrm{H}$ & -0.718633 & -1.555584 & -2.779629 \\
\hline $\mathrm{H}$ & 1.766495 & 1.756787 & -2.020937 \\
\hline $\mathrm{C}$ & -2.446469 & -2.423496 & -0.080143 \\
\hline $\mathrm{C}$ & -2.308480 & 2.374915 & -0.702575 \\
\hline $\mathrm{C}$ & 2.154318 & 2.338329 & 1.160414 \\
\hline $\mathrm{C}$ & 2.376797 & -2.390450 & 0.155964 \\
\hline C & -2.834726 & -1.109355 & -0.304363 \\
\hline C & -4.186111 & -0.692971 & -0.618577 \\
\hline $\mathrm{C}$ & -4.127277 & 0.655258 & -0.888547 \\
\hline $\mathrm{C}$ & -2.746835 & 1.058769 & -0.691363 \\
\hline $\mathrm{C}$ & -5.240736 & 1.581102 & -1.259496 \\
\hline $\mathrm{C}$ & -5.374676 & -1.599660 & -0.633784 \\
\hline C & -1.067534 & 2.806995 & -0.240230 \\
\hline C & -0.729405 & 4.189037 & 0.020590 \\
\hline $\mathrm{C}$ & 0.507470 & 4.181913 & 0.646081 \\
\hline $\mathrm{C}$ & 0.922738 & 2.784480 & 0.703221 \\
\hline C & -1.580215 & 5.362189 & -0.337724 \\
\hline C & 1.310685 & 5.289391 & 1.131312 \\
\hline C & 0.900853 & 6.535511 & 1.442967 \\
\hline $\mathrm{C}$ & 2.640783 & 1.040866 & 1.009482 \\
\hline . & 3.997569 & 0.635349 & 1.288248 \\
\hline
\end{tabular}




\begin{tabular}{|c|c|c|c|}
\hline $\mathrm{C}$ & 4.095270 & -0.694574 & 0.901055 \\
\hline $\mathrm{C}$ & 2.762240 & -1.085123 & 0.457510 \\
\hline C & 5.044533 & 1.499108 & 1.910958 \\
\hline $\mathrm{C}$ & 5.235621 & -1.591290 & 0.956613 \\
\hline C & 6.541534 & -1.256620 & 0.968470 \\
\hline $\mathrm{C}$ & 1.069121 & -2.827436 & -0.003061 \\
\hline C & 0.671444 & -4.229212 & -0.020020 \\
\hline C & -0.701524 & -4.243337 & 0.006024 \\
\hline $\mathrm{C}$ & -1.124892 & -2.851773 & -0.011322 \\
\hline $\mathrm{C}$ & 1.620759 & -5.383228 & -0.012375 \\
\hline $\mathrm{C}$ & -1.630160 & -5.413955 & 0.039795 \\
\hline $\mathrm{N}$ & -1.970408 & -0.030876 & -0.359247 \\
\hline $\mathrm{N}$ & -0.048388 & 1.970074 & 0.156736 \\
\hline $\mathrm{N}$ & 1.913861 & -0.009537 & 0.493162 \\
\hline $\mathrm{N}$ & -0.038247 & -2.013601 & -0.033500 \\
\hline $\mathrm{Fe}$ & -0.048765 & -0.014606 & 0.059910 \\
\hline $\mathrm{H}$ & -3.227886 & -3.183317 & -0.046780 \\
\hline $\mathrm{H}$ & -3.037485 & 3.137994 & -0.974746 \\
\hline $\mathrm{H}$ & 2.830935 & 3.074688 & 1.591700 \\
\hline $\mathrm{H}$ & 3.151508 & -3.157056 & 0.162010 \\
\hline $\mathrm{H}$ & -6.156436 & 1.027317 & -1.504999 \\
\hline $\mathrm{H}$ & -4.982090 & 2.199100 & -2.133840 \\
\hline $\mathrm{H}$ & -5.484751 & 2.272597 & -0.436054 \\
\hline $\mathrm{H}$ & -5.573981 & -2.018675 & 0.365401 \\
\hline $\mathrm{H}$ & -5.231763 & -2.450159 & -1.319256 \\
\hline $\mathrm{H}$ & -6.280602 & -1.067290 & -0.952542 \\
\hline $\mathrm{H}$ & -2.192926 & 5.702020 & 0.514846 \\
\hline $\mathrm{H}$ & -2.268487 & 5.128150 & -1.161838 \\
\hline $\mathrm{H}$ & -0.957322 & 6.215556 & -0.646494 \\
\hline $\mathrm{H}$ & 2.376150 & 5.073629 & 1.266435 \\
\hline $\mathrm{H}$ & -0.142295 & 6.845600 & 1.392446 \\
\hline $\mathrm{H}$ & 1.616025 & 7.281020 & 1.792861 \\
\hline $\mathrm{H}$ & 4.599722 & 2.287720 & 2.534052 \\
\hline $\mathrm{H}$ & 5.681804 & 1.994793 & 1.158623 \\
\hline $\mathrm{H}$ & 5.709849 & 0.899247 & 2.549906 \\
\hline $\mathrm{H}$ & 4.997252 & -2.660084 & 0.986481 \\
\hline $\mathrm{H}$ & 6.886417 & -0.225048 & 0.897381 \\
\hline $\mathrm{H}$ & 7.311285 & -2.027037 & 1.029789 \\
\hline $\mathrm{H}$ & 2.185762 & -5.437705 & 0.932875 \\
\hline $\mathrm{H}$ & 2.358554 & -5.311404 & -0.827769 \\
\hline $\mathrm{H}$ & 1.090714 & -6.337475 & -0.130528 \\
\hline $\mathrm{H}$ & -1.079460 & -6.362875 & -0.000677 \\
\hline $\mathrm{H}$ & -2.333571 & -5.405399 & -0.809147 \\
\hline $\mathrm{H}$ & -2.234956 & -5.423653 & 0.961108 \\
\hline $\mathrm{C}$ & -0.372904 & -0.187711 & 1.915963 \\
\hline $\mathrm{N}$ & -0.586568 & -0.288400 & 3.066273 \\
\hline \multicolumn{4}{|c|}{ His-heme-CN, $164.4^{\circ} \alpha$-meso-Fe- $\gamma$-meso angle } \\
\hline $\mathrm{C}$ & 0.522518 & -0.342945 & -4.176487 \\
\hline $\mathrm{N}$ & 1.308512 & 0.754398 & -3.872319 \\
\hline C & -0.019623 & -0.746993 & -2.981707 \\
\hline $\mathrm{C}$ & 1.222929 & 0.980439 & -2.534730 \\
\hline
\end{tabular}




\begin{tabular}{|c|c|c|c|}
\hline $\mathrm{N}$ & 0.423022 & 0.084048 & -1.972452 \\
\hline $\mathrm{H}$ & 0.425910 & -0.724494 & -5.186059 \\
\hline $\mathrm{H}$ & 1.856622 & 1.299049 & -4.529910 \\
\hline $\mathrm{H}$ & -0.689616 & -1.573711 & -2.774284 \\
\hline $\mathrm{H}$ & 1.738273 & 1.780665 & -2.014509 \\
\hline C & -2.445950 & -2.428652 & -0.102778 \\
\hline $\mathrm{C}$ & -2.317916 & 2.376272 & -0.678511 \\
\hline C & 2.162417 & 2.343117 & 1.140349 \\
\hline C & 2.374700 & -2.395981 & 0.179004 \\
\hline C & -2.835315 & -1.113053 & -0.316526 \\
\hline C & -4.187413 & -0.697165 & -0.627329 \\
\hline C & -4.132351 & 0.654836 & -0.879051 \\
\hline C & -2.753156 & 1.059175 & -0.676232 \\
\hline C & -5.248048 & 1.581977 & -1.239787 \\
\hline C & -5.373375 & -1.606912 & -0.655206 \\
\hline C & -1.074293 & 2.808814 & -0.224616 \\
\hline C & -0.733757 & 4.191575 & 0.030407 \\
\hline C & 0.511758 & 4.185769 & 0.638062 \\
\hline C & 0.926985 & 2.788003 & 0.692781 \\
\hline $\mathrm{C}$ & -1.589285 & 5.364416 & -0.317471 \\
\hline C & 1.322516 & 5.293995 & 1.108662 \\
\hline C & 0.919576 & 6.543257 & 1.417039 \\
\hline C & 2.645084 & 1.043381 & 1.000323 \\
\hline C & 4.001301 & 0.638120 & 1.281450 \\
\hline C & 4.094151 & -0.697276 & 0.912288 \\
\hline C & 2.760126 & -1.089352 & 0.474444 \\
\hline C & 5.052352 & 1.506643 & 1.890456 \\
\hline C & 5.231652 & -1.596941 & 0.980690 \\
\hline $\mathrm{C}$ & 6.538476 & -1.265915 & 0.979546 \\
\hline C & 1.068068 & -2.831839 & 0.010661 \\
\hline C & 0.670651 & -4.233737 & -0.013715 \\
\hline C & -0.702285 & -4.247922 & -0.005626 \\
\hline C & -1.125112 & -2.856058 & -0.023776 \\
\hline C & 1.620180 & -5.387491 & 0.001882 \\
\hline C & -1.631275 & -5.418575 & 0.013926 \\
\hline $\mathrm{N}$ & -1.973941 & -0.032146 & -0.356364 \\
\hline $\mathrm{N}$ & -0.050385 & 1.972314 & 0.160886 \\
\hline $\mathrm{N}$ & 1.913781 & -0.011751 & 0.499371 \\
\hline $\mathrm{N}$ & -0.038382 & -2.017269 & -0.029896 \\
\hline $\mathrm{Fe}$ & -0.049828 & -0.016389 & 0.066060 \\
\hline $\mathrm{H}$ & -3.226692 & -3.189610 & -0.082338 \\
\hline $\mathrm{H}$ & -3.050382 & 3.138996 & -0.942209 \\
\hline $\mathrm{H}$ & 2.844256 & 3.081779 & 1.559323 \\
\hline $\mathrm{H}$ & 3.148885 & -3.162972 & 0.191477 \\
\hline $\mathrm{H}$ & -6.163303 & 1.028678 & -1.488032 \\
\hline $\mathrm{H}$ & -4.992304 & 2.207640 & -2.109529 \\
\hline $\mathrm{H}$ & -5.491527 & 2.266217 & -0.410160 \\
\hline $\mathrm{H}$ & -5.572366 & -2.039124 & 0.338464 \\
\hline $\mathrm{H}$ & -5.227804 & -2.448324 & -1.351271 \\
\hline $\mathrm{H}$ & -6.280535 & -1.072915 & -0.967720 \\
\hline $\mathrm{H}$ & -2.180473 & 5.713351 & 0.546566 \\
\hline $\mathrm{H}$ & -2.297407 & 5.126421 & -1.12327 \\
\hline
\end{tabular}




\begin{tabular}{|c|c|c|c|}
\hline $\mathrm{H}$ & & & \\
\hline $\mathrm{H}$ & & & \\
\hline & 2.388824 & 5.076358 & 1.233807 \\
\hline $\mathrm{H}$ & -0.123062 & 6.856201 & 1.374987 \\
\hline $\mathrm{H}$ & 1.640466 & 7.288642 & 1.755247 \\
\hline $\mathrm{H}$ & 4.611666 & 2.303084 & 2.506498 \\
\hline $\mathrm{H}$ & 5.687041 & 1.992936 & 1.129878 \\
\hline $\mathrm{H}$ & 5.719305 & 0.912583 & 2.533069 \\
\hline $\mathrm{H}$ & 4.989824 & -2.663991 & 1.034503 \\
\hline $\mathrm{H}$ & 6.885665 & -0.237006 & 0.884790 \\
\hline $\mathrm{H}$ & 7.306382 & -2.037064 & 1.053747 \\
\hline $\mathrm{H}$ & 2.176351 & -5.442677 & 0.952258 \\
\hline $\mathrm{H}$ & 2.365569 & -5.314371 & -0.806491 \\
\hline $\mathrm{H}$ & 1.091576 & -6.341760 & -0.122438 \\
\hline $\mathrm{H}$ & -1.079914 & -6.367395 & -0.019149 \\
\hline $\mathrm{H}$ & -2.322612 & -5.409791 & -0.844855 \\
\hline $\mathrm{H}$ & -2.249019 & -5.429066 & 0.926655 \\
\hline $\mathrm{C}$ & -0.379981 & -0.185284 & 1.921220 \\
\hline $\mathrm{N}$ & -0.597336 & -0.283686 & 3.071051 \\
\hline His $-\mathrm{H}$ & $\mathrm{CN}, 165.4^{\circ} \alpha-\mathrm{me}$ & e- $\gamma$-meso angle & \\
\hline $\mathrm{C}$ & 0.525142 & -0.344372 & -4.168933 \\
\hline $\mathrm{N}$ & 1.301940 & 0.759805 & -3.865864 \\
\hline C & -0.015055 & -0.750611 & -2.974044 \\
\hline $\mathrm{C}$ & 1.214314 & 0.986578 & -2.528578 \\
\hline $\mathrm{N}$ & 0.421148 & 0.084678 & -1.965434 \\
\hline $\mathrm{H}$ & 0.432173 & -0.728089 & -5.178028 \\
\hline $\mathrm{H}$ & 1.845860 & 1.308118 & -4.523894 \\
\hline $\mathrm{H}$ & -0.678881 & -1.582152 & -2.766139 \\
\hline $\mathrm{H}$ & 1.723151 & 1.791515 & -2.009231 \\
\hline $\mathrm{C}$ & -2.446318 & -2.433661 & -0.124012 \\
\hline $\mathrm{C}$ & -2.324359 & 2.376623 & -0.657851 \\
\hline $\mathrm{C}$ & 2.171437 & 2.348736 & 1.123398 \\
\hline C & 2.371972 & -2.398683 & 0.199500 \\
\hline $\mathrm{C}$ & -2.836031 & -1.116565 & -0.326663 \\
\hline $\mathrm{C}$ & -4.188067 & -0.700175 & -0.636939 \\
\hline $\mathrm{C}$ & -4.135150 & 0.654602 & -0.872965 \\
\hline $\mathrm{C}$ & -2.757153 & 1.059026 & -0.662942 \\
\hline $\mathrm{C}$ & -5.251813 & 1.583493 & -1.226253 \\
\hline $\mathrm{C}$ & -5.372309 & -1.611666 & -0.676936 \\
\hline $\mathrm{C}$ & -1.079073 & 2.809220 & -0.209578 \\
\hline $\mathrm{C}$ & -0.736806 & 4.192673 & 0.039144 \\
\hline C & 0.515540 & 4.188778 & 0.632375 \\
\hline $\mathrm{C}$ & 0.931377 & 2.791206 & 0.686722 \\
\hline $\mathrm{C}$ & -1.596337 & 5.364703 & -0.301696 \\
\hline $\mathrm{C}$ & 1.331162 & 5.298216 & 1.091449 \\
\hline $\mathrm{C}$ & 0.931810 & 6.548732 & 1.399523 \\
\hline C & 2.649271 & 1.046671 & 0.992960 \\
\hline $\mathrm{C}$ & 4.005383 & 0.641169 & 1.274037 \\
\hline $\mathrm{C}$ & 4.093546 & -0.698401 & 0.919875 \\
\hline $\mathrm{C}$ & 2.757805 & -1.091238 & 0.488584 \\
\hline C & 5.060212 & 1.513542 & 1.870991 \\
\hline $\mathrm{C}$ & 5.228680 & -1.600525 & 0.995891 \\
\hline
\end{tabular}




$\begin{array}{lrrr}\mathrm{C} & 6.536348 & -1.273159 & 0.986712 \\ \mathrm{C} & 1.066336 & -2.833577 & 0.024578 \\ \mathrm{C} & 0.670170 & -4.235518 & -0.006971 \\ \mathrm{C} & -0.702597 & -4.250480 & -0.014933 \\ \mathrm{C} & -1.125938 & -2.858880 & -0.033366 \\ \mathrm{C} & 1.620185 & -5.388726 & 0.016509 \\ \mathrm{C} & -1.631132 & -5.421608 & -0.008014 \\ \mathrm{~N} & -1.976183 & -0.033848 & -0.352191 \\ \mathrm{~N} & -0.051361 & 1.973387 & 0.167815 \\ \mathrm{~N} & 1.913141 & -0.011788 & 0.505046 \\ \mathrm{~N} & -0.039772 & -2.018666 & -0.024738 \\ \mathrm{Fe} & -0.051599 & -0.017076 & 0.074264 \\ \mathrm{H} & -3.226074 & -3.195776 & -0.113902 \\ \mathrm{H} & -3.058768 & 3.139301 & -0.916143 \\ \mathrm{H} & 2.857264 & 3.088893 & 1.533060 \\ \mathrm{H} & 3.145509 & -3.166198 & 0.217402 \\ \mathrm{H} & -6.166014 & 1.031238 & -1.480683 \\ \mathrm{H} & -4.996005 & 2.217592 & -2.089811 \\ \mathrm{H} & -5.497136 & 2.259616 & -0.390559 \\ \mathrm{H} & -5.572469 & -2.054830 & 0.311682 \\ \mathrm{H} & -5.224067 & -2.445347 & -1.381717 \\ \mathrm{H} & -6.279793 & -1.075825 & -0.985372 \\ \mathrm{H} & -2.175365 & 5.717394 & 0.569035 \\ \mathrm{H} & -2.315512 & 5.124234 & -1.096799 \\ \mathrm{H} & -0.982578 & 6.212165 & -0.643700 \\ \mathrm{H} & 2.398532 & 5.080483 & 1.207168 \\ \mathrm{H} & -0.110959 & 6.862257 & 1.366237 \\ \mathrm{H} & 1.656301 & 7.294741 & 1.728527 \\ \mathrm{H} & 4.623114 & 2.316975 & 2.480452 \\ \mathrm{H} & 5.693418 & 1.990866 & 1.103551 \\ \mathrm{H} & 5.727979 & 0.924466 & 2.517338 \\ \mathrm{H} & 4.983926 & -2.666050 & 1.064046 \\ \mathrm{H} & 6.885990 & -0.246472 & 0.877917 \\ \mathrm{H} & 7.302399 & -2.045417 & 1.068258 \\ \mathrm{H} & 2.166756 & -5.444799 & 0.972338 \\ \mathrm{H} & 2.373673 & -5.313872 & -0.784151 \\ \mathrm{H} & 1.093498 & -6.343162 & -0.114565 \\ \mathrm{H} & -1.078855 & -6.370050 & -0.035661 \\ \mathrm{H} & -2.312237 & -5.412191 & -0.874902 \\ \mathrm{H} & -2.259658 & -5.433576 & 0.897333 \\ \mathrm{C} & -0.383354 & -0.185701 & 1.928982 \\ \mathrm{~N} & -0.601712 & -0.283931 & 3.078707\end{array}$

His-heme-CN, $166.3^{\circ} \alpha$-meso-Fe- $\gamma$-meso angle

$\begin{array}{llrl}\mathrm{C} & 0.723504 & -0.429154 & -4.147280 \\ \mathrm{~N} & 1.158124 & 0.862033 & -3.908712 \\ \mathrm{C} & 0.276580 & -0.894561 & -2.935384 \\ \mathrm{C} & 0.965099 & 1.139251 & -2.591718 \\ \mathrm{~N} & 0.432336 & 0.089350 & -1.979385 \\ \mathrm{H} & 0.772281 & -0.885066 & -5.129193 \\ \mathrm{H} & 1.553899 & 1.496874 & -4.594125\end{array}$




\begin{tabular}{|c|c|c|c|}
\hline $\mathrm{H}$ & -0.142039 & -1.861184 & -2.680746 \\
\hline $\mathrm{H}$ & 1.214698 & 2.083854 & -2.121712 \\
\hline $\mathrm{C}$ & -2.439204 & -2.427937 & -0.156474 \\
\hline $\mathrm{C}$ & -2.340677 & 2.393596 & -0.617534 \\
\hline $\mathrm{C}$ & 2.182094 & 2.338812 & 1.078433 \\
\hline $\mathrm{C}$ & 2.372090 & -2.428330 & 0.227812 \\
\hline C & -2.827722 & -1.107686 & -0.355187 \\
\hline C & -4.181716 & -0.692862 & -0.656937 \\
\hline C & -4.138590 & 0.668771 & -0.862871 \\
\hline C & -2.765815 & 1.073982 & -0.643986 \\
\hline $\mathrm{C}$ & -5.261160 & 1.597499 & -1.198835 \\
\hline $\mathrm{C}$ & -5.360715 & -1.609539 & -0.716253 \\
\hline C & -1.090880 & 2.822589 & -0.181164 \\
\hline $\mathrm{C}$ & -0.742185 & 4.204567 & 0.070767 \\
\hline C & 0.525370 & 4.191680 & 0.628190 \\
\hline C & 0.938550 & 2.789226 & 0.659818 \\
\hline C & -1.610275 & 5.381016 & -0.232521 \\
\hline C & 1.356970 & 5.293514 & 1.075710 \\
\hline C & 0.971831 & 6.545090 & 1.397954 \\
\hline C & 2.650167 & 1.032594 & 0.962077 \\
\hline C & 4.005186 & 0.629191 & 1.245319 \\
\hline C & 4.089670 & -0.720334 & 0.922749 \\
\hline C & 2.755991 & -1.117761 & 0.504272 \\
\hline $\mathrm{C}$ & 5.066305 & 1.508695 & 1.819181 \\
\hline C & 5.222531 & -1.623669 & 1.021516 \\
\hline C & 6.530787 & -1.300920 & 0.989203 \\
\hline C & 1.066047 & -2.853591 & 0.049750 \\
\hline C & 0.659734 & -4.252396 & 0.016958 \\
\hline C & -0.711020 & -4.254651 & -0.018163 \\
\hline C & -1.124536 & -2.857195 & -0.044900 \\
\hline C & 1.601830 & -5.411781 & 0.062826 \\
\hline C & -1.649609 & -5.417152 & -0.026208 \\
\hline $\mathrm{N}$ & -1.975977 & -0.023964 & -0.360943 \\
\hline $\mathrm{N}$ & -0.053954 & 1.980257 & 0.158448 \\
\hline $\mathrm{N}$ & 1.908513 & -0.036792 & 0.502902 \\
\hline $\mathrm{N}$ & -0.031873 & -2.023862 & -0.017974 \\
\hline $\mathrm{Fe}$ & -0.043324 & -0.027205 & 0.065258 \\
\hline $\mathrm{H}$ & -3.220815 & -3.188254 & -0.160965 \\
\hline $\mathrm{H}$ & -3.083197 & 3.156496 & -0.850961 \\
\hline $\mathrm{H}$ & 2.879360 & 3.076632 & 1.472959 \\
\hline $\mathrm{H}$ & 3.143761 & -3.197142 & 0.254449 \\
\hline $\mathrm{H}$ & -6.179153 & 1.044485 & -1.437194 \\
\hline $\mathrm{H}$ & -5.020811 & 2.229556 & -2.068425 \\
\hline $\mathrm{H}$ & -5.493270 & 2.275130 & -0.360707 \\
\hline $\mathrm{H}$ & -5.541011 & -2.094888 & 0.256281 \\
\hline $\mathrm{H}$ & -5.217589 & -2.412494 & -1.456966 \\
\hline $\mathrm{H}$ & -6.276598 & -1.068143 & -0.987662 \\
\hline $\mathrm{H}$ & -2.155631 & 5.731306 & 0.660607 \\
\hline $\mathrm{H}$ & -2.359538 & 5.147719 & -1.001556 \\
\hline $\mathrm{H}$ & -1.007600 & 6.228806 & -0.593223 \\
\hline $\mathrm{H}$ & 2.425109 & 5.068563 & 1.168183 \\
\hline $\mathrm{H}$ & -0.069491 & 6.864827 & 1.386951 \\
\hline
\end{tabular}




$\begin{array}{lrrr}\mathrm{H} & 1.707395 & 7.285016 & 1.715964 \\ \mathrm{H} & 4.636544 & 2.322376 & 2.420075 \\ \mathrm{H} & 5.690900 & 1.972736 & 1.036805 \\ \mathrm{H} & 5.740299 & 0.928119 & 2.466504 \\ \mathrm{H} & 4.974347 & -2.684846 & 1.131423 \\ \mathrm{H} & 6.882008 & -0.280023 & 0.838807 \\ \mathrm{H} & 7.295422 & -2.071979 & 1.092490 \\ \mathrm{H} & 2.140907 & -5.459714 & 1.023259 \\ \mathrm{H} & 2.362067 & -5.352576 & -0.732987 \\ \mathrm{H} & 1.069574 & -6.364094 & -0.060937 \\ \mathrm{H} & -1.106193 & -6.370716 & -0.000483 \\ \mathrm{H} & -2.287122 & -5.425173 & -0.925757 \\ \mathrm{H} & -2.322414 & -5.398252 & 0.846671 \\ \mathrm{C} & -0.437225 & -0.132439 & 1.913707 \\ \mathrm{~N} & -0.688980 & -0.196851 & 3.058992\end{array}$

His-heme-CN, $167.3^{\circ} \alpha$-meso-Fe- $\gamma$-meso angle

$\begin{array}{lrrr}\mathrm{C} & 0.721824 & -0.433765 & -4.141462 \\ \mathrm{~N} & 1.148393 & 0.860803 & -3.906719 \\ \mathrm{C} & 0.279682 & -0.899114 & -2.927771 \\ \mathrm{C} & 0.955375 & 1.140024 & -2.590101 \\ \mathrm{~N} & 0.430475 & 0.088192 & -1.974473 \\ \mathrm{H} & 0.772133 & -0.891740 & -5.122341 \\ \mathrm{H} & 1.539293 & 1.496447 & -4.594184 \\ \mathrm{H} & -0.132397 & -1.867736 & -2.670168 \\ \mathrm{H} & 1.200155 & 2.087360 & -2.123069 \\ \mathrm{C} & -2.437749 & -2.431310 & -0.179750 \\ \mathrm{C} & -2.347353 & 2.394834 & -0.595580 \\ \mathrm{C} & 2.190512 & 2.344636 & 1.058907 \\ \mathrm{C} & 2.369672 & -2.430533 & 0.249846 \\ \mathrm{C} & -2.827008 & -1.109757 & -0.367027 \\ \mathrm{C} & -4.181389 & -0.694977 & -0.666653 \\ \mathrm{C} & -4.141337 & 0.669160 & -0.855236 \\ \mathrm{C} & -2.769650 & 1.074628 & -0.630347 \\ \mathrm{C} & -5.265701 & 1.599136 & -1.181582 \\ \mathrm{C} & -5.358036 & -1.613746 & -0.738729 \\ \mathrm{C} & -1.095413 & 2.824039 & -0.166673 \\ \mathrm{C} & -0.745114 & 4.206599 & 0.079950 \\ \mathrm{C} & 0.529399 & 4.195428 & 0.620842 \\ \mathrm{C} & 0.943161 & 2.793114 & 0.650504 \\ \mathrm{C} & -1.617441 & 5.382344 & -0.213656 \\ \mathrm{C} & 1.366282 & 5.298358 & 1.055764 \\ \mathrm{C} & 0.984385 & 6.549585 & 1.383244 \\ \mathrm{C} & 2.654609 & 1.036376 & 0.953078 \\ \mathrm{C} & 4.009191 & 0.632962 & 1.237843 \\ \mathrm{C} & 4.089008 & -0.720704 & 0.932294 \\ \mathrm{C} & 2.754074 & -1.118984 & 0.519376 \\ \mathrm{C} & 5.074130 & 1.516363 & 1.798528 \\ \mathrm{C} & 5.219095 & -1.626277 & 1.041718 \\ \mathrm{C} & 6.528345 & -1.308198 & 1.004312 \\ \mathrm{C} & 1.064753 & -2.855070 & 0.063348 \\ \mathrm{C} & 0.659628 & -4.254045 & 0.023042\end{array}$




$\begin{array}{lrrr}\mathrm{C} & -0.710397 & -4.256887 & -0.029265 \\ \mathrm{C} & -1.123982 & -2.859462 & -0.056419 \\ \mathrm{C} & 1.601819 & -5.413029 & 0.077195 \\ \mathrm{C} & -1.648457 & -5.419693 & -0.050573 \\ \mathrm{~N} & -1.977419 & -0.024311 & -0.357943 \\ \mathrm{~N} & -0.054609 & 1.982293 & 0.162927 \\ \mathrm{~N} & 1.908463 & -0.036184 & 0.507928 \\ \mathrm{~N} & -0.032360 & -2.025284 & -0.013561 \\ \mathrm{Fe} & -0.044220 & -0.027111 & 0.071372 \\ \mathrm{H} & -3.218329 & -3.192450 & -0.196936 \\ \mathrm{H} & -3.092455 & 3.157470 & -0.821454 \\ \mathrm{H} & 2.891934 & 3.084302 & 1.442433 \\ \mathrm{H} & 3.140594 & -3.199815 & 0.282959 \\ \mathrm{H} & -6.181808 & 1.046673 & -1.428396 \\ \mathrm{H} & -5.025470 & 2.242435 & -2.042862 \\ \mathrm{H} & -5.501013 & 2.265775 & -0.335575 \\ \mathrm{H} & -5.537239 & -2.112844 & 0.227057 \\ \mathrm{H} & -5.213085 & -2.406188 & -1.490377 \\ \mathrm{H} & -6.275186 & -1.070815 & -1.002697 \\ \mathrm{H} & -2.153746 & 5.731511 & 0.685359 \\ \mathrm{H} & -2.374503 & 5.149132 & -0.975037 \\ \mathrm{H} & -1.019261 & 6.230876 & -0.579938 \\ \mathrm{H} & 2.435966 & 5.074692 & 1.132558 \\ \mathrm{H} & -0.057459 & 6.867838 & 1.387698 \\ \mathrm{H} & 1.723559 & 7.290591 & 1.690176 \\ \mathrm{H} & 4.648073 & 2.339816 & 2.388532 \\ \mathrm{H} & 5.699442 & 1.967282 & 1.009118 \\ \mathrm{H} & 5.746611 & 0.942170 & 2.453110 \\ \mathrm{H} & 4.967605 & -2.685059 & 1.166029 \\ \mathrm{H} & 6.882795 & -0.290543 & 0.840289 \\ \mathrm{H} & 7.290447 & -2.080420 & 1.117276 \\ \mathrm{H} & 2.129628 & -5.462888 & 1.043713 \\ \mathrm{H} & 2.371230 & -5.351320 & -0.709542 \\ \mathrm{H} & 1.071568 & -6.365316 & -0.055225 \\ \mathrm{H} & -1.104876 & -6.373073 & -0.021721 \\ \mathrm{H} & -2.276606 & -5.425536 & -0.956656 \\ \mathrm{H} & -2.330273 & -5.403477 & 0.815373 \\ \mathrm{C} & -0.439894 & -0.131349 & 1.919004 \\ \mathrm{~N} & -0.692102 & -0.195202 & 3.064243 \\ & & & \end{array}$

His-heme-CN, $168.2^{\circ} \alpha$-meso-Fe- $\gamma$-meso angle

$\begin{array}{lrrr}\mathrm{C} & 0.727474 & -0.439275 & -4.135987 \\ \mathrm{~N} & 1.139860 & 0.860598 & -3.905100 \\ \mathrm{C} & 0.289587 & -0.905525 & -2.921107 \\ \mathrm{C} & 0.942830 & 1.141932 & -2.589575 \\ \mathrm{~N} & 0.428932 & 0.086448 & -1.970878 \\ \mathrm{H} & 0.783447 & -0.899796 & -5.115367 \\ \mathrm{H} & 1.524593 & 1.498163 & -4.594269 \\ \mathrm{H} & -0.112082 & -1.877741 & -2.660678 \\ \mathrm{H} & 1.177175 & 2.093263 & -2.125314\end{array}$




\begin{tabular}{|c|c|c|c|}
\hline C & -2.436153 & -2.434015 & -0.204321 \\
\hline C & -2.354687 & 2.396433 & -0.572857 \\
\hline C & 2.198845 & 2.350078 & 1.037698 \\
\hline C & 2.366694 & -2.433163 & 0.27266 \\
\hline C & -2.826354 & -1.111397 & -0.38084 \\
\hline C & -4.181168 & -0.696760 & -0.67821 \\
\hline C & -4.144577 & 0.669819 & -0.84846 \\
\hline C & -2.774081 & 1.075650 & -0.61725 \\
\hline C & -5.270876 & 1.600960 & -1.16464 \\
\hline C & -5.355248 & -1.617651 & -0.76396 \\
\hline C & -1.100510 & 2.825595 & -0.15162 \\
\hline C & -0.748697 & 4.208619 & 0.0908 \\
\hline $\mathrm{C}$ & 0.532970 & 4.198870 & 0.61398 \\
\hline C & 0.947635 & 2.796774 & 0.64012 \\
\hline C & -1.625613 & 5.383800 & -0.1909 \\
\hline C & 1.375188 & 5.302838 & 1.0359 \\
\hline C & 0.996343 & 6.552724 & 1.3719 \\
\hline C & 2.659229 & 1.039991 & 0.9419 \\
\hline C & 4.013360 & 0.636558 & 1.2281 \\
\hline $\mathrm{C}$ & 4.088280 & -0.721231 & 0.9404 \\
\hline C & 2.751984 & -1.120438 & 0.5335 \\
\hline $\mathrm{C}$ & 5.082319 & 1.523784 & 1.77501 \\
\hline C & 5.215616 & -1.628945 & 1.0604 \\
\hline C & 6.525759 & -1.315587 & 1.0159 \\
\hline C & 1.062884 & -2.856965 & 0.07806 \\
\hline C & 0.658479 & -4.256038 & 0.0319 \\
\hline C & -0.710550 & -4.258952 & -0.03860 \\
\hline C & -1.123642 & -2.861412 & -0.06830 \\
\hline C & 1.600281 & -5.414838 & 0.0963 \\
\hline C & -1.648284 & -5.421780 & -0.07258 \\
\hline $\mathrm{N}$ & -1.979315 & -0.024124 & -0.3564 \\
\hline $\mathrm{N}$ & -0.055381 & 1.984346 & 0.16613 \\
\hline $\mathrm{N}$ & 1.908587 & -0.035698 & 0.5112 \\
\hline $\mathrm{N}$ & -0.032876 & -2.026776 & -0.00993 \\
\hline $\mathrm{Fe}$ & -0.044965 & -0.026762 & 0.07613 \\
\hline $\mathrm{H}$ & -3.215622 & -3.195895 & -0.23400 \\
\hline $\mathrm{H}$ & -3.102461 & 3.159005 & -0.78993 \\
\hline $\mathrm{H}$ & 2.904134 & 3.091518 & 1.4105 \\
\hline $\mathrm{H}$ & 3.136638 & -3.203025 & 0.3134 \\
\hline $\mathrm{H}$ & -6.185920 & 1.049290 & -1.41715 \\
\hline $\mathrm{H}$ & -5.032060 & 2.253797 & -2.0190 \\
\hline $\mathrm{H}$ & -5.507293 & 2.258062 & -0.3115 \\
\hline $\mathrm{H}$ & -5.532564 & -2.132239 & 0.19404 \\
\hline $\mathrm{H}$ & -5.208707 & -2.397913 & -1.52799 \\
\hline $\mathrm{H}$ & -6.274003 & -1.073122 & -1.01890 \\
\hline $\mathrm{H}$ & -2.152743 & 5.730027 & 0.71459 \\
\hline $\mathrm{H}$ & -2.390532 & 5.151790 & -0.9448 \\
\hline $\mathrm{H}$ & -1.032258 & 6.233934 & -0.5612 \\
\hline $\mathrm{H}$ & 2.446548 & 5.081192 & 1.0936 \\
\hline $\mathrm{H}$ & -0.046088 & 6.868331 & 1.3954 \\
\hline $\mathrm{H}$ & 1.739033 & 7.295053 & 1.6669 \\
\hline $\mathrm{H}$ & 4.660205 & 2.355517 & 2.3560 \\
\hline
\end{tabular}




$\begin{array}{rrrr}\mathrm{H} & 5.706432 & 1.963333 & 0.978305 \\ \mathrm{H} & 5.755003 & 0.955681 & 2.434665 \\ \mathrm{H} & 4.960888 & -2.684924 & 1.200661 \\ \mathrm{H} & 6.882998 & -0.301529 & 0.836559 \\ \mathrm{H} & 7.285595 & -2.088577 & 1.138588 \\ \mathrm{H} & 2.116586 & -5.465254 & 1.068925 \\ \mathrm{H} & 2.378941 & -5.352062 & -0.681176 \\ \mathrm{H} & 1.071901 & -6.367143 & -0.043378 \\ \mathrm{H} & -1.104917 & -6.375080 & -0.037597 \\ \mathrm{H} & -2.265248 & -5.427119 & -0.986278 \\ \mathrm{H} & -2.340678 & -5.406292 & 0.784974 \\ \mathrm{C} & -0.442232 & -0.128548 & 1.923196 \\ \mathrm{~N} & -0.694899 & -0.191150 & 3.068432\end{array}$

His-heme-CN, $169.2^{\circ} \alpha$-meso-Fe- $\gamma$-meso angle

$\begin{array}{lrrr}\mathrm{C} & 0.734527 & -0.445201 & -4.130658 \\ \mathrm{~N} & 1.132983 & 0.859674 & -3.903469 \\ \mathrm{C} & 0.300050 & -0.912125 & -2.914816 \\ \mathrm{C} & 0.931213 & 1.143197 & -2.589169 \\ \mathrm{~N} & 0.427595 & 0.084388 & -1.967657 \\ \mathrm{H} & 0.796638 & -0.908269 & -5.108468 \\ \mathrm{H} & 1.512097 & 1.498952 & -4.594169 \\ \mathrm{H} & -0.091667 & -1.887670 & -2.651752 \\ \mathrm{H} & 1.155117 & 2.098351 & -2.127617 \\ \mathrm{C} & -2.434332 & -2.436315 & -0.229203 \\ \mathrm{C} & -2.362181 & 2.397937 & -0.549696 \\ \mathrm{C} & 2.206968 & 2.355373 & 1.015807 \\ \mathrm{C} & 2.363438 & -2.435608 & 0.295723 \\ \mathrm{C} & -2.825613 & -1.112768 & -0.395066 \\ \mathrm{C} & -4.180922 & -0.698321 & -0.690123 \\ \mathrm{C} & -4.147956 & 0.670485 & -0.841599 \\ \mathrm{C} & -2.778657 & 1.076665 & -0.604088 \\ \mathrm{C} & -5.276250 & 1.602704 & -1.147267 \\ \mathrm{C} & -5.352230 & -1.621335 & -0.790078 \\ \mathrm{C} & -1.105710 & 2.827040 & -0.136372 \\ \mathrm{C} & -0.752453 & 4.210481 & 0.102123 \\ \mathrm{C} & 0.536265 & 4.202148 & 0.607079 \\ \mathrm{C} & 0.951924 & 2.800323 & 0.629288 \\ \mathrm{C} & -1.633952 & 5.385094 & -0.167245 \\ \mathrm{C} & 1.383639 & 5.307184 & 1.016027 \\ \mathrm{C} & 1.007786 & 6.555282 & 1.361798 \\ \mathrm{C} & 2.663762 & 1.043576 & 0.930090 \\ \mathrm{C} & 4.017466 & 0.640132 & 1.217674 \\ \mathrm{C} & 4.087449 & -0.721567 & 0.948089 \\ \mathrm{C} & 2.749741 & -1.121701 & 0.547476 \\ \mathrm{C} & 5.090417 & 1.531051 & 1.750663 \\ \mathrm{C} & 5.212035 & -1.631274 & 1.078844 \\ \mathrm{C} & 6.523029 & -1.322813 & 1.026733 \\ \mathrm{C} & 1.060790 & -2.858716 & 0.093043 \\ \mathrm{C} & 0.657043 & -4.257837 & 0.041353 \\ \mathrm{C} & -0.710749 & -4.260691 & -0.047554 \\ \mathrm{C} & -1.123236 & -2.863039 & -0.080210\end{array}$




\begin{tabular}{|c|c|c|c|}
\hline C & 1.598174 & -5.416561 & 0.11651 \\
\hline C & -1.648124 & -5.423415 & -0.09417 \\
\hline $\mathrm{N}$ & -1.981263 & -0.023828 & -0.35528 \\
\hline $\mathrm{N}$ & -0.056226 & 1.986340 & 0.16898 \\
\hline $\mathrm{N}$ & 1.908623 & -0.035114 & 0.51414 \\
\hline $\mathrm{N}$ & -0.033451 & -2.028078 & -0.00643 \\
\hline $\mathrm{Fe}$ & -0.045663 & -0.026319 & 0.08048 \\
\hline $\mathrm{H}$ & -3.212558 & -3.198867 & -0.27139 \\
\hline $\mathrm{H}$ & -3.112630 & 3.160448 & -0.75757 \\
\hline $\mathrm{H}$ & 2.916005 & 3.098563 & 1.37800 \\
\hline $\mathrm{H}$ & 3.132351 & -3.206014 & 0.34449 \\
\hline $\mathrm{H}$ & -6.190901 & 1.051902 & $-1.4030 s$ \\
\hline $\mathrm{H}$ & -5.039958 & 2.263548 & -1.99619 \\
\hline $\mathrm{H}$ & -5.512050 & 2.251658 & $-0.2877 \mathrm{~s}$ \\
\hline $\mathrm{H}$ & -5.526666 & -2.152934 & 0.1591 \\
\hline $\mathrm{H}$ & -5.204470 & -2.387862 & -1.56771 \\
\hline $\mathrm{H}$ & -6.272966 & -1.075274 & -1.03438 \\
\hline $\mathrm{H}$ & -2.152331 & 5.727528 & 0.7447 \\
\hline $\mathrm{H}$ & -2.406274 & 5.154694 & -0.91403 \\
\hline $\mathrm{H}$ & -1.045445 & 6.237235 & -0.54047 \\
\hline $\mathrm{H}$ & 2.456467 & 5.087980 & 1.05317 \\
\hline $\mathrm{H}$ & -0.035005 & 6.867630 & 1.40581 \\
\hline $\mathrm{H}$ & 1.753721 & 7.299122 & 1.6445 \\
\hline $\mathrm{H}$ & 4.672244 & 2.370242 & 2.32376 \\
\hline $\mathrm{H}$ & 5.712506 & 1.960148 & 0.94672 \\
\hline $\mathrm{H}$ & 5.764079 & 0.968813 & 2.41430 \\
\hline $\mathrm{H}$ & 4.954100 & -2.684112 & 1.23563 \\
\hline $\mathrm{H}$ & 6.882934 & -0.312689 & 0.83129 \\
\hline $\mathrm{H}$ & 7.280661 & -2.096352 & 1.15921 \\
\hline $\mathrm{H}$ & 2.102868 & -5.467188 & 1.09514 \\
\hline $\mathrm{H}$ & 2.385998 & -5.353173 & $-0.6515 s$ \\
\hline $\mathrm{H}$ & 1.071632 & -6.368869 & -0.03000 \\
\hline $\mathrm{H}$ & -1.105252 & -6.376701 & -0.05176 \\
\hline $\mathrm{H}$ & -2.252894 & -5.428858 & -1.01594 \\
\hline $\mathrm{H}$ & -2.351836 & -5.407849 & 0.75414 \\
\hline C & -0.444601 & -0.125219 & 1.9270 \\
\hline $\mathrm{N}$ & -0 . & -0.186334 & 3 \\
\hline
\end{tabular}

His-heme-CN, $170.2^{\circ} \alpha$-meso-Fe- $\gamma$-meso angle

$\begin{array}{rrrr}\mathrm{C} & 0.742420 & -0.451207 & -4.125342 \\ \mathrm{~N} & 1.126705 & 0.858576 & -3.901926 \\ \mathrm{C} & 0.310861 & -0.918600 & -2.908644 \\ \mathrm{C} & 0.919998 & 1.144287 & -2.588903 \\ \mathrm{~N} & 0.426507 & 0.082327 & -1.964601 \\ \mathrm{H} & 0.810986 & -0.916855 & -5.101493 \\ \mathrm{H} & 1.500263 & 1.499479 & -4.594152 \\ \mathrm{H} & -0.071036 & -1.897311 & -2.642968 \\ \mathrm{H} & 1.133387 & 2.103165 & -2.130104 \\ \mathrm{C} & -2.432255 & -2.438320 & -0.254323 \\ \mathrm{C} & -2.369735 & 2.399251 & -0.526094 \\ \mathrm{C} & 2.214826 & 2.360471 & 0.993503\end{array}$




\begin{tabular}{|c|c|c|c|}
\hline $\mathrm{C}$ & 2.359913 & -2.437824 & 0.318923 \\
\hline $\mathrm{C}$ & -2.824767 & -1.113963 & -0.409452 \\
\hline $\mathrm{C}$ & -4.180623 & -0.699710 & -0.701998 \\
\hline $\mathrm{C}$ & -4.151419 & 0.671090 & -0.834319 \\
\hline $\mathrm{C}$ & -2.783303 & 1.077583 & -0.590654 \\
\hline $\mathrm{C}$ & -5.281776 & 1.604302 & -1.129034 \\
\hline $\mathrm{C}$ & -5.349000 & -1.624769 & -0.816667 \\
\hline $\mathrm{C}$ & -1.110902 & 2.828336 & -0.120991 \\
\hline C & -0.756188 & 4.212194 & 0.113460 \\
\hline C & 0.539427 & 4.205277 & 0.599862 \\
\hline C & 0.956055 & 2.803740 & 0.618047 \\
\hline $\mathrm{C}$ & -1.642169 & 5.386219 & -0.143212 \\
\hline C & 1.391836 & 5.311429 & 0.995406 \\
\hline $\mathrm{C}$ & 1.019151 & 6.557510 & 1.351620 \\
\hline $\mathrm{C}$ & 2.668138 & 1.047059 & 0.917940 \\
\hline $\mathrm{C}$ & 4.021402 & 0.643600 & 1.207032 \\
\hline C & 4.086461 & -0.721766 & 0.955737 \\
\hline C & 2.747328 & -1.122772 & 0.561384 \\
\hline C & 5.098285 & 1.538062 & 1.726079 \\
\hline $\mathrm{C}$ & 5.208285 & -1.633329 & 1.097311 \\
\hline $\mathrm{C}$ & 6.520107 & -1.329925 & 1.037650 \\
\hline $\mathrm{C}$ & 1.058515 & -2.860306 & 0.108026 \\
\hline $\mathrm{C}$ & 0.655446 & -4.259453 & 0.050954 \\
\hline $\mathrm{C}$ & -0.710870 & -4.262213 & -0.056484 \\
\hline $\mathrm{C}$ & -1.122714 & -2.864462 & -0.092313 \\
\hline $\mathrm{C}$ & 1.595704 & -5.418142 & 0.137204 \\
\hline $\mathrm{C}$ & -1.647743 & -5.424817 & -0.115801 \\
\hline $\mathrm{N}$ & -1.983194 & -0.023516 & -0.354191 \\
\hline $\mathrm{N}$ & -0.057130 & 1.988240 & 0.171637 \\
\hline $\mathrm{N}$ & 1.908566 & -0.034460 & 0.516821 \\
\hline $\mathrm{N}$ & -0.034091 & -2.029231 & -0.003199 \\
\hline $\mathrm{Fe}$ & -0.046408 & -0.025870 & 0.084569 \\
\hline $\mathrm{H}$ & -3.209103 & -3.201477 & -0.309027 \\
\hline $\mathrm{H}$ & -3.122856 & 3.161664 & -0.724467 \\
\hline $\mathrm{H}$ & 2.927494 & 3.105401 & 1.344913 \\
\hline $\mathrm{H}$ & 3.127740 & -3.208735 & 0.375892 \\
\hline $\mathrm{H}$ & -6.196616 & 1.054412 & -1.386138 \\
\hline $\mathrm{H}$ & -5.048999 & 2.271965 & -1.973583 \\
\hline $\mathrm{H}$ & -5.515429 & 2.246215 & -0.263740 \\
\hline $\mathrm{H}$ & -5.519722 & -2.174643 & 0.122807 \\
\hline $\mathrm{H}$ & -5.200361 & -2.376101 & -1.608892 \\
\hline $\mathrm{H}$ & -6.272019 & -1.077323 & -1.048924 \\
\hline $\mathrm{H}$ & -2.151981 & 5.724436 & 0.775131 \\
\hline $\mathrm{H}$ & -2.421635 & 5.157617 & -0.883122 \\
\hline $\mathrm{H}$ & -1.058507 & 6.240552 & -0.518861 \\
\hline $\mathrm{H}$ & 2.465801 & 5.094913 & 1.011099 \\
\hline $\mathrm{H}$ & -0.023630 & 6.866257 & 1.417152 \\
\hline $\mathrm{H}$ & 1.768132 & 7.302995 & 1.621715 \\
\hline $\mathrm{H}$ & 4.684010 & 2.384328 & 2.291496 \\
\hline $\mathrm{H}$ & 5.718036 & 1.957049 & 0.915052 \\
\hline $\mathrm{H}$ & 5.773218 & 0.981629 & 2.393268 \\
\hline $\mathrm{H}$ & 4.947148 & -2.682762 & 1.270572 \\
\hline
\end{tabular}




$\begin{array}{lrl}\mathrm{H} & 6.882643 & -0.323982 \\ \mathrm{H} & 7.275536 & -2.103874 \\ \mathrm{H} & 2.088573 & -5.468824 \\ \mathrm{H} & 2.392691 & -5.354340 \\ \mathrm{H} & 1.071051 & -6.370449 \\ \mathrm{H} & -1.105524 & -6.378095 \\ \mathrm{H} & -2.239780 & -5.430588 \\ \mathrm{H} & -2.363031 & -5.408887 \\ \mathrm{C} & -0.447053 & -0.122011 \\ \mathrm{~N} & -0.701104 & -0.181684\end{array}$

$$
\begin{array}{r}
0.826245 \\
1.180001 \\
1.121774 \\
-0.621327 \\
-0.016038 \\
-0.065442 \\
-1.045758 \\
0.722789 \\
1.930611 \\
3.075749
\end{array}
$$

His-heme-CN, $171.1^{\circ} \alpha$-meso-Fe- $\gamma$-meso angle

$\begin{array}{lrrr}\mathrm{C} & 0.750996 & -0.456816 & -4.120062 \\ \mathrm{~N} & 1.120799 & 0.857765 & -3.900352 \\ \mathrm{C} & 0.322393 & -0.924806 & -2.902556 \\ \mathrm{C} & 0.908800 & 1.145551 & -2.588664 \\ \mathrm{~N} & 0.425626 & 0.080428 & -1.961622 \\ \mathrm{H} & 0.826289 & -0.924928 & -5.094538 \\ \mathrm{H} & 1.488761 & 1.500328 & -4.594041 \\ \mathrm{H} & -0.049371 & -1.906693 & -2.634298 \\ \mathrm{H} & 1.111347 & 2.108078 & -2.132612 \\ \mathrm{C} & -2.429945 & -2.440111 & -0.279614 \\ \mathrm{C} & -2.377315 & 2.400295 & -0.501984 \\ \mathrm{C} & 2.222392 & 2.365327 & 0.970918 \\ \mathrm{C} & 2.356071 & -2.439855 & 0.342318 \\ \mathrm{C} & -2.823835 & -1.115066 & -0.423944 \\ \mathrm{C} & -4.180259 & -0.701016 & -0.713862 \\ \mathrm{C} & -4.154923 & 0.671543 & -0.826622 \\ \mathrm{C} & -2.787989 & 1.078319 & -0.576899 \\ \mathrm{C} & -5.287418 & 1.605654 & -1.109913 \\ \mathrm{C} & -5.345584 & -1.627965 & -0.843796 \\ \mathrm{C} & -1.116074 & 2.829424 & -0.105413 \\ \mathrm{C} & -0.759864 & 4.213716 & 0.124855 \\ \mathrm{C} & 0.542469 & 4.208232 & 0.592356 \\ \mathrm{C} & 0.960015 & 2.806982 & 0.606513 \\ \mathrm{C} & -1.650194 & 5.387139 & -0.118918 \\ \mathrm{C} & 1.399818 & 5.315568 & 0.974013 \\ \mathrm{C} & 1.030586 & 6.559517 & 1.340992 \\ \mathrm{C} & 2.672349 & 1.050400 & 0.905567 \\ \mathrm{C} & 4.025165 & 0.646942 & 1.196201 \\ \mathrm{C} & 4.085312 & -0.721852 & 0.963327 \\ \mathrm{C} & 2.744731 & -1.123691 & 0.575270 \\ \mathrm{C} & 5.105956 & 1.544788 & 1.701203 \\ \mathrm{C} & 5.204379 & -1.635117 & 1.115693 \\ \mathrm{C} & 6.517020 & -1.336897 & 1.048904 \\ \mathrm{C} & 1.056012 & -2.861789 & 0.123078 \\ \mathrm{C} & 0.653630 & -4.260961 & 0.060752 \\ \mathrm{C} & -0.710952 & -4.263612 & -0.065421 \\ \mathrm{C} & -1.122105 & -2.865769 & -0.104569 \\ \mathrm{C} & 1.592796 & -5.419654 & 0.158455 \\ \mathrm{C} & -1.647161 & -5.426084 & -0.137568 \\ \mathrm{~N} & -1.985108 & -0.023268 & -0.353100\end{array}$




\begin{tabular}{|c|c|c|c|}
\hline $\mathrm{N}$ & -0.058109 & 1.989992 & 0.174195 \\
\hline $\mathrm{N}$ & 1.908410 & -0.033779 & 0.519337 \\
\hline $\mathrm{N}$ & -0.034832 & -2.030308 & -0.000162 \\
\hline $\mathrm{Fe}$ & -0.047257 & -0.025478 & 0.088480 \\
\hline $\mathrm{H}$ & -3.205284 & -3.203806 & -0.346842 \\
\hline $\mathrm{H}$ & -3.133095 & 3.162563 & -0.690604 \\
\hline $\mathrm{H}$ & 2.938570 & 3.111987 & 1.311440 \\
\hline $\mathrm{H}$ & 3.122748 & -3.211238 & 0.407690 \\
\hline $\mathrm{H}$ & -6.202966 & 1.056678 & -1.366432 \\
\hline $\mathrm{H}$ & -5.059087 & 2.279120 & -1.951072 \\
\hline $\mathrm{H}$ & -5.517505 & 2.241504 & -0.239245 \\
\hline $\mathrm{H}$ & -5.512326 & -2.196726 & 0.085102 \\
\hline $\mathrm{H}$ & -5.196117 & -2.363079 & -1.650997 \\
\hline $\mathrm{H}$ & -6.271006 & -1.079376 & -1.063406 \\
\hline $\mathrm{H}$ & -2.151516 & 5.720871 & 0.805692 \\
\hline $\mathrm{H}$ & -2.436626 & 5.160462 & -0.852049 \\
\hline $\mathrm{H}$ & -1.071355 & 6.243772 & -0.496611 \\
\hline $\mathrm{H}$ & 2.474517 & 5.101927 & 0.967434 \\
\hline $\mathrm{H}$ & -0.011733 & 6.864446 & 1.428819 \\
\hline $\mathrm{H}$ & 1.782432 & 7.306780 & 1.597903 \\
\hline $\mathrm{H}$ & 4.695585 & 2.398007 & 2.258907 \\
\hline $\mathrm{H}$ & 5.723289 & 1.953636 & 0.883197 \\
\hline $\mathrm{H}$ & 5.782236 & 0.994195 & 2.371826 \\
\hline $\mathrm{H}$ & 4.940055 & -2.680962 & 1.304930 \\
\hline $\mathrm{H}$ & 6.882241 & -0.335311 & 0.822068 \\
\hline $\mathrm{H}$ & 7.270205 & -2.111189 & 1.200988 \\
\hline $\mathrm{H}$ & 2.073443 & -5.470327 & 1.148990 \\
\hline $\mathrm{H}$ & 2.399080 & -5.355558 & -0.590116 \\
\hline $\mathrm{H}$ & 1.070117 & -6.371946 & -0.001564 \\
\hline $\mathrm{H}$ & -1.105720 & -6.379366 & -0.079367 \\
\hline $\mathrm{H}$ & -2.226310 & -5.432046 & -1.075563 \\
\hline $\mathrm{H}$ & -2.373924 & -5.409880 & 0.691101 \\
\hline C & -0.449496 & -0.119112 & 1.934030 \\
\hline $\mathrm{N}$ & -0.704306 & -0.177470 & 3.079092 \\
\hline \multicolumn{4}{|c|}{ His-heme- $\mathrm{CN}, 172.1^{\circ} \alpha$-meso-Fe- $\gamma$-meso angle } \\
\hline $\mathrm{C}$ & 0.759941 & -0.462134 & -4.114957 \\
\hline $\mathrm{N}$ & 1.114395 & 0.857322 & -3.899161 \\
\hline $\mathrm{C}$ & 0.334514 & -0.930768 & -2.896591 \\
\hline $\mathrm{C}$ & 0.897079 & 1.147099 & -2.588815 \\
\hline $\mathrm{N}$ & 0.424865 & 0.078741 & -1.958880 \\
\hline $\mathrm{H}$ & 0.842236 & -0.932702 & -5.087683 \\
\hline $\mathrm{H}$ & 1.476312 & 1.501631 & -4.594415 \\
\hline $\mathrm{H}$ & -0.026566 & -1.915881 & -2.625613 \\
\hline $\mathrm{H}$ & 1.088274 & 2.113300 & -2.135657 \\
\hline $\mathrm{C}$ & -2.427332 & -2.441645 & -0.305140 \\
\hline $\mathrm{C}$ & -2.384880 & 2.401078 & -0.477411 \\
\hline $\mathrm{C}$ & 2.229717 & 2.369913 & 0.947977 \\
\hline $\mathrm{C}$ & 2.352011 & -2.441709 & 0.365899 \\
\hline $\mathrm{C}$ & -2.822757 & -1.116028 & -0.438511 \\
\hline $\mathrm{C}$ & -4.179784 & -0.702176 & -0.725722 \\
\hline $\mathrm{C}$ & -4.158414 & 0.671874 & -0.81855 \\
\hline
\end{tabular}




\begin{tabular}{|c|c|c|c|}
\hline $\mathrm{C}$ & -2.792671 & 1.078892 & -0.562796 \\
\hline $\mathrm{C}$ & -5.293111 & 1.606770 & -1.090015 \\
\hline $\mathrm{C}$ & -5.341960 & -1.630812 & -0.871367 \\
\hline $\mathrm{C}$ & -1.121193 & 2.830314 & -0.089648 \\
\hline $\mathrm{C}$ & -0.763445 & 4.215033 & 0.136285 \\
\hline $\mathrm{C}$ & 0.545418 & 4.210955 & 0.584577 \\
\hline $\mathrm{C}$ & 0.963815 & 2.810000 & 0.594683 \\
\hline $\mathrm{C}$ & -1.657971 & 5.387841 & -0.094424 \\
\hline C & 1.407583 & 5.319469 & 0.952076 \\
\hline $\mathrm{C}$ & 1.042149 & 6.561214 & 1.330014 \\
\hline $\mathrm{C}$ & 2.676440 & 1.053572 & 0.892924 \\
\hline $\mathrm{C}$ & 4.028791 & 0.650122 & 1.185283 \\
\hline C & 4.084078 & -0.721849 & 0.970966 \\
\hline $\mathrm{C}$ & 2.742038 & -1.124474 & 0.589168 \\
\hline $\mathrm{C}$ & 5.113425 & 1.551191 & 1.676221 \\
\hline $\mathrm{C}$ & 5.200383 & -1.636658 & 1.134184 \\
\hline C & 6.513847 & -1.343740 & 1.060822 \\
\hline C & 1.053371 & -2.863154 & 0.138195 \\
\hline C & 0.651689 & -4.262328 & 0.070679 \\
\hline $\mathrm{C}$ & -0.710903 & -4.264821 & -0.074398 \\
\hline $\mathrm{C}$ & -1.121325 & -2.866900 & -0.117037 \\
\hline $\mathrm{C}$ & 1.589508 & -5.421083 & 0.180171 \\
\hline $\mathrm{C}$ & -1.646277 & -5.427157 & -0.159480 \\
\hline $\mathrm{N}$ & -1.986956 & -0.023055 & -0.351925 \\
\hline $\mathrm{N}$ & -0.059158 & 1.991579 & 0.176676 \\
\hline $\mathrm{N}$ & 1.908240 & -0.033097 & 0.521639 \\
\hline $\mathrm{N}$ & -0.035586 & -2.031272 & 0.002689 \\
\hline $\mathrm{Fe}$ & -0.048147 & -0.025134 & 0.092143 \\
\hline $\mathrm{H}$ & -3.201012 & -3.205815 & -0.384942 \\
\hline $\mathrm{H}$ & -3.143289 & 3.163136 & -0.656085 \\
\hline $\mathrm{H}$ & 2.949295 & 3.118300 & 1.277445 \\
\hline $\mathrm{H}$ & 3.117449 & -3.213542 & 0.439923 \\
\hline $\mathrm{H}$ & -6.209747 & 1.058669 & -1.344477 \\
\hline $\mathrm{H}$ & -5.070001 & 2.285295 & -1.928528 \\
\hline $\mathrm{H}$ & -5.518520 & 2.237285 & -0.214308 \\
\hline $\mathrm{H}$ & -5.505445 & -2.217779 & 0.046732 \\
\hline $\mathrm{H}$ & -5.191063 & -2.349787 & -1.692758 \\
\hline $\mathrm{H}$ & -6.269610 & -1.081474 & -1.079330 \\
\hline $\mathrm{H}$ & -2.150745 & 5.716976 & 0.836377 \\
\hline $\mathrm{H}$ & -2.451296 & 5.163121 & -0.820735 \\
\hline $\mathrm{H}$ & -1.083971 & 6.246800 & -0.474025 \\
\hline $\mathrm{H}$ & 2.482547 & 5.108763 & 0.922825 \\
\hline $\mathrm{H}$ & 0.000816 & 6.862248 & 1.440565 \\
\hline $\mathrm{H}$ & 1.796677 & 7.310280 & 1.573499 \\
\hline $\mathrm{H}$ & 4.706915 & 2.411427 & 2.225846 \\
\hline $\mathrm{H}$ & 5.728533 & 1.949595 & 0.851413 \\
\hline $\mathrm{H}$ & 5.790883 & 1.006569 & 2.350483 \\
\hline $\mathrm{H}$ & 4.932868 & -2.678756 & 1.338883 \\
\hline $\mathrm{H}$ & 6.881830 & -0.346666 & 0.819118 \\
\hline $\mathrm{H}$ & 7.264731 & -2.118314 & 1.222583 \\
\hline $\mathrm{H}$ & 2.057417 & -5.471797 & 1.176730 \\
\hline $\mathrm{H}$ & 2.405296 & -5.356751 & -0.557953 \\
\hline
\end{tabular}




$\begin{array}{rrrr}\mathrm{H} & 1.068897 & -6.373323 & 0.013161 \\ \mathrm{H} & -1.105686 & -6.380458 & -0.093986 \\ \mathrm{H} & -2.212712 & -5.432939 & -1.105165 \\ \mathrm{H} & -2.384131 & -5.411006 & 0.659334 \\ \mathrm{C} & -0.451617 & -0.116583 & 1.937272 \\ \mathrm{~N} & -0.707020 & -0.173774 & 3.082281\end{array}$

His-heme-CN, $173.1^{\circ} \alpha$-meso-Fe- $\gamma$-meso angle

$\begin{array}{lrrr}\mathrm{C} & 0.774665 & -0.468458 & -4.108782 \\ \mathrm{~N} & 1.104644 & 0.858243 & -3.898759 \\ \mathrm{C} & 0.354057 & -0.938475 & -2.889265 \\ \mathrm{C} & 0.879203 & 1.150443 & -2.590357 \\ \mathrm{~N} & 0.424399 & 0.076964 & -1.956201 \\ \mathrm{H} & 0.868118 & -0.942330 & -5.078891 \\ \mathrm{H} & 1.456841 & 1.505451 & -4.596314 \\ \mathrm{H} & 0.009767 & -1.928449 & -2.614256 \\ \mathrm{H} & 1.052448 & 2.121937 & -2.141360 \\ \mathrm{C} & -2.424537 & -2.442721 & -0.331214 \\ \mathrm{C} & -2.392587 & 2.401766 & -0.451890 \\ \mathrm{C} & 2.236819 & 2.374113 & 0.924633 \\ \mathrm{C} & 2.347476 & -2.443472 & 0.389756 \\ \mathrm{C} & -2.821710 & -1.116661 & -0.453429 \\ \mathrm{C} & -4.179435 & -0.702994 & -0.737499 \\ \mathrm{C} & -4.162187 & 0.672296 & -0.809661 \\ \mathrm{C} & -2.797566 & 1.079462 & -0.548075 \\ \mathrm{C} & -5.299172 & 1.607953 & -1.068368 \\ \mathrm{C} & -5.338361 & -1.633058 & -0.899416 \\ \mathrm{C} & -1.126246 & 2.831138 & -0.073766 \\ \mathrm{C} & -0.766793 & 4.216272 & 0.147685 \\ \mathrm{C} & 0.548526 & 4.213509 & 0.576068 \\ \mathrm{C} & 0.967717 & 2.812848 & 0.581895 \\ \mathrm{C} & -1.665425 & 5.388484 & -0.069372 \\ \mathrm{C} & 1.415589 & 5.323281 & 0.928428 \\ \mathrm{C} & 1.054374 & 6.562304 & 1.318911 \\ \mathrm{C} & 2.680406 & 1.056447 & 0.880084 \\ \mathrm{C} & 4.032206 & 0.652946 & 1.174327 \\ \mathrm{C} & 4.082558 & -0.721967 & 0.978794 \\ \mathrm{C} & 2.739044 & -1.125236 & 0.603154 \\ \mathrm{C} & 5.120781 & 1.557025 & 1.650875 \\ \mathrm{C} & 5.196011 & -1.638248 & 1.152978 \\ \mathrm{C} & 6.510325 & -1.350916 & 1.073327 \\ \mathrm{C} & 1.050415 & -2.864494 & 0.153049 \\ \mathrm{C} & 0.649336 & -4.263638 & 0.080484 \\ \mathrm{C} & -0.710982 & -4.265861 & -0.084025 \\ \mathrm{C} & -1.120515 & -2.867844 & -0.130414 \\ \mathrm{C} & 1.585421 & -5.422568 & 0.202597 \\ \mathrm{C} & -1.645386 & -5.427995 & -0.182304 \\ \mathrm{~N} & -1.988941 & -0.022723 & -0.350799 \\ \mathrm{~N} & -0.060092 & 1.993147 & 0.178477 \\ \mathrm{~N} & 1.907924 & -0.032467 & 0.523875 \\ \mathrm{~N} & -0.036434 & -2.032207 & 0.004631 \\ \mathrm{Fe} & -0.049095 & -0.024781 & 0.095383\end{array}$




\begin{tabular}{|c|c|c|c|}
\hline $\mathrm{H}$ & & & \\
\hline & -3.196446 & -3.207262 & -0.423791 \\
\hline $\mathrm{H}$ & -3.153655 & 3.163610 & -0.619975 \\
\hline $\mathrm{H}$ & 2.959696 & 3.124269 & 1.242798 \\
\hline $\mathrm{H}$ & 3.111579 & -3.215715 & 0.472790 \\
\hline $\mathrm{H}$ & -6.218085 & 1.060679 & -1.316277 \\
\hline $\mathrm{H}$ & -5.083465 & 2.289082 & -1.906775 \\
\hline $\mathrm{H}$ & -5.516592 & 2.235687 & -0.188710 \\
\hline $\mathrm{H}$ & -5.499703 & -2.237302 & 0.007805 \\
\hline $\mathrm{H}$ & -5.185214 & -2.336237 & -1.733986 \\
\hline $\mathrm{H}$ & -6.267970 & -1.083305 & -1.097235 \\
\hline $\mathrm{H}$ & -2.150310 & 5.711922 & 0.867520 \\
\hline $\mathrm{H}$ & -2.465064 & 5.166296 & -0.789567 \\
\hline $\mathrm{H}$ & -1.096128 & 6.250251 & -0.449490 \\
\hline $\mathrm{H}$ & 2.490316 & 5.116057 & 0.873974 \\
\hline $\mathrm{H}$ & 0.014621 & 6.858701 & 1.454643 \\
\hline $\mathrm{H}$ & 1.811375 & 7.313507 & 1.547754 \\
\hline $\mathrm{H}$ & 4.718283 & 2.424381 & 2.192142 \\
\hline $\mathrm{H}$ & 5.733616 & 1.944632 & 0.819259 \\
\hline $\mathrm{H}$ & 5.799401 & 1.018516 & 2.328832 \\
\hline $\mathrm{H}$ & 4.925204 & -2.676371 & 1.372909 \\
\hline $\mathrm{H}$ & 6.881241 & -0.358597 & 0.817034 \\
\hline $\mathrm{H}$ & 7.258755 & -2.125787 & 1.244763 \\
\hline $\mathrm{H}$ & 2.039501 & -5.473398 & 1.205467 \\
\hline $\mathrm{H}$ & 2.411320 & -5.358124 & -0.524105 \\
\hline $\mathrm{H}$ & 1.067011 & -6.374680 & 0.028087 \\
\hline $\mathrm{H}$ & -1.105688 & -6.381357 & -0.110457 \\
\hline $\mathrm{H}$ & -2.199475 & -5.432920 & -1.135227 \\
\hline $\mathrm{H}$ & -2.393791 & -5.412475 & 0.626890 \\
\hline C & -0.453941 & -0.113795 & 1.940121 \\
\hline $\mathrm{N}$ & -0.710030 & -0.169695 & 3.085059 \\
\hline His $-\mathrm{r}$ & $\mathrm{N}, 174.0^{\circ} \alpha-\mathrm{me}$ & $\mathrm{e}-\gamma$-meso angle & \\
\hline $\mathrm{C}$ & 0.781175 & -0.472456 & -4.104646 \\
\hline $\mathrm{N}$ & 1.099669 & 0.857568 & -3.897804 \\
\hline $\mathrm{C}$ & 0.362987 & -0.942695 & -2.884399 \\
\hline $\mathrm{C}$ & 0.870422 & 1.151263 & -2.590427 \\
\hline $\mathrm{N}$ & 0.423864 & 0.075726 & -1.953894 \\
\hline $\mathrm{H}$ & 0.879725 & -0.948129 & -5.073367 \\
\hline $\mathrm{H}$ & 1.447203 & 1.505902 & -4.596655 \\
\hline $\mathrm{H}$ & 0.026595 & -1.934780 & -2.607234 \\
\hline $\mathrm{H}$ & 1.035198 & 2.125360 & -2.143868 \\
\hline C & -2.421241 & -2.443897 & -0.357175 \\
\hline $\mathrm{C}$ & -2.399817 & 2.401835 & -0.426702 \\
\hline C & 2.243650 & 2.378280 & 0.901509 \\
\hline $\mathrm{C}$ & 2.342979 & -2.444737 & 0.413556 \\
\hline $\mathrm{C}$ & -2.820168 & -1.117490 & -0.468174 \\
\hline $\mathrm{C}$ & -4.178509 & -0.704006 & -0.749355 \\
\hline C & -4.165376 & 0.672208 & -0.800987 \\
\hline C & -2.801952 & 1.079524 & -0.533507 \\
\hline C & -5.304730 & 1.608291 & -1.047177 \\
\hline $\mathrm{C}$ & -5.334321 & -1.635157 & -0.926952 \\
\hline $\mathrm{C}$ & -1.131098 & 2.831433 & -0.057577 \\
\hline
\end{tabular}




\begin{tabular}{|c|c|c|c|}
\hline C & -0.770184 & 4.217044 & 0.159197 \\
\hline C & 0.551054 & 4.215774 & 0.568358 \\
\hline C & 0.971066 & 2.815422 & 0.570291 \\
\hline C & -1.672674 & 5.388520 & -0.045213 \\
\hline C & 1.422356 & 5.326738 & 0.906619 \\
\hline C & 1.065529 & 6.563900 & 1.306788 \\
\hline $\mathrm{C}$ & 2.684279 & 1.059420 & 0.867121 \\
\hline C & 4.035616 & 0.655926 & 1.163160 \\
\hline C & 4.081173 & -0.721638 & 0.986293 \\
\hline C & 2.736161 & -1.125563 & 0.616964 \\
\hline C & 5.127943 & 1.562807 & 1.625729 \\
\hline $\mathrm{C}$ & 5.191851 & -1.639183 & 1.171265 \\
\hline C & 6.506996 & -1.357247 & 1.086370 \\
\hline C & 1.047468 & -2.865376 & 0.168464 \\
\hline C & 0.647295 & -4.264533 & 0.090635 \\
\hline C & -0.710456 & -4.266701 & -0.093181 \\
\hline $\mathrm{C}$ & -1.119334 & -2.868667 & -0.143030 \\
\hline $\mathrm{C}$ & 1.581617 & -5.423541 & 0.225046 \\
\hline $\mathrm{C}$ & -1.643456 & -5.428812 & -0.204912 \\
\hline $\mathrm{N}$ & -1.990387 & -0.022734 & -0.349678 \\
\hline $\mathrm{N}$ & -0.061257 & 1.994254 & 0.181429 \\
\hline $\mathrm{N}$ & 1.907704 & -0.031619 & 0.525797 \\
\hline $\mathrm{N}$ & -0.037275 & -2.032811 & 0.007578 \\
\hline $\mathrm{Fe}$ & -0.050057 & -0.024499 & 0.098805 \\
\hline $\mathrm{H}$ & -3.191237 & -3.208755 & -0.462228 \\
\hline $\mathrm{H}$ & -3.163393 & 3.163318 & -0.584768 \\
\hline $\mathrm{H}$ & 2.969638 & 3.130075 & 1.208578 \\
\hline $\mathrm{H}$ & 3.105645 & -3.217386 & 0.505518 \\
\hline $\mathrm{H}$ & -6.224860 & 1.061683 & -1.292008 \\
\hline $\mathrm{H}$ & -5.095039 & 2.294263 & -1.883202 \\
\hline $\mathrm{H}$ & -5.516698 & 2.230892 & -0.162612 \\
\hline $\mathrm{H}$ & -5.495584 & -2.253402 & -0.029178 \\
\hline $\mathrm{H}$ & -5.177613 & -2.325236 & -1.771744 \\
\hline $\mathrm{H}$ & -6.265205 & -1.085305 & -1.118302 \\
\hline $\mathrm{H}$ & -2.148801 & 5.707912 & 0.897506 \\
\hline $\mathrm{H}$ & -2.479107 & 5.167776 & -0.758279 \\
\hline $\mathrm{H}$ & -1.108278 & 6.252353 & -0.427743 \\
\hline $\mathrm{H}$ & 2.496352 & 5.122168 & 0.830827 \\
\hline $\mathrm{H}$ & 0.027786 & 6.856907 & 1.463847 \\
\hline $\mathrm{H}$ & 1.824747 & 7.316772 & 1.522484 \\
\hline $\mathrm{H}$ & 4.729249 & 2.437684 & 2.157520 \\
\hline $\mathrm{H}$ & 5.739545 & 1.938701 & 0.787838 \\
\hline $\mathrm{H}$ & 5.806891 & 1.030704 & 2.308370 \\
\hline $\mathrm{H}$ & 4.917834 & -2.673358 & 1.405270 \\
\hline $\mathrm{H}$ & 6.880830 & -0.369597 & 0.816701 \\
\hline $\mathrm{H}$ & 7.253034 & -2.132301 & 1.267169 \\
\hline $\mathrm{H}$ & 2.021709 & -5.474734 & 1.234066 \\
\hline $\mathrm{H}$ & 2.417518 & -5.358646 & -0.490003 \\
\hline $\mathrm{H}$ & 1.065700 & -6.375564 & 0.042763 \\
\hline $\mathrm{H}$ & -1.104440 & -6.382150 & -0.127691 \\
\hline $\mathrm{H}$ & -2.185588 & -5.432385 & -1.164649 \\
\hline $\mathrm{H}$ & -2.401910 & -5.414691 & 0.594893 \\
\hline
\end{tabular}




$\begin{array}{llll}\mathrm{C} & -0.455749 & -0.112197 & 1.943178 \\ \mathrm{~N} & -0.712291 & -0.167390 & 3.088066\end{array}$

His-heme- $\mathrm{CN}, 175.2^{\circ} \alpha$-meso-Fe- $\gamma$-meso angle, fully relaxed

$\begin{array}{lrrr}\mathrm{C} & -0.526013502 & -0.386936080 & -4.259386177 \\ \mathrm{~N} & 0.667907450 & 0.307765434 & -4.188153506 \\ \mathrm{C} & -0.916672009 & -0.577694391 & -2.957131401 \\ \mathrm{C} & 0.960616663 & 0.516734134 & -2.877222996 \\ \mathrm{~N} & 0.015283342 & -0.011504560 & -2.109867822 \\ \mathrm{C} & -3.417292710 & 0.000451963 & 0.139815593 \\ \mathrm{C} & 0.011798677 & 3.417107748 & -0.098724345 \\ \mathrm{C} & 3.415904490 & -0.000000000 & 0.148790967 \\ \mathrm{C} & -0.016729334 & -3.416948960 & -0.080637733 \\ \mathrm{C} & -2.771902547 & 1.228972414 & 0.069391352 \\ \mathrm{C} & -3.459609086 & 2.502558750 & 0.068754396 \\ \mathrm{C} & -2.486215912 & 3.472252449 & -0.025334099 \\ \mathrm{C} & -1.217085233 & 2.776499787 & -0.067115579 \\ \mathrm{C} & -2.648126565 & 4.958254846 & -0.052253680 \\ \mathrm{C} & -4.941467101 & 2.672704985 & 0.167093757 \\ \mathrm{C} & 1.250300240 & 2.790578001 & -0.039513643 \\ \mathrm{C} & 2.516262534 & 3.491057893 & 0.024487118 \\ \mathrm{C} & 3.495049699 & 2.518658075 & 0.119971852 \\ \mathrm{C} & 2.793079016 & 1.236531008 & 0.092990888 \\ \mathrm{C} & 2.688955264 & 4.973070354 & -0.023299571 \\ \mathrm{C} & 4.938576261 & 2.654148464 & 0.195742773 \\ \mathrm{C} & 5.647167836 & 3.724097733 & 0.609228119 \\ \mathrm{C} & 2.777647936 & -1.234400220 & 0.091142961 \\ \mathrm{C} & 3.470396652 & -2.497733537 & 0.106649733 \\ \mathrm{C} & 2.494140283 & -3.481442327 & 0.005741745 \\ \mathrm{C} & 1.221371138 & -2.783736271 & -0.037689215 \\ \mathrm{C} & 4.944611801 & -2.674202165 & 0.264991381 \\ \mathrm{C} & 2.638438083 & -4.926630298 & -0.003787491 \\ \mathrm{C} & 3.716603843 & -5.637442063 & -0.389193069 \\ \mathrm{C} & -1.244499839 & -2.780561354 & -0.029256847 \\ \mathrm{C} & -2.521028273 & -3.480192024 & 0.039074254 \\ \mathrm{C} & -3.486206506 & -2.512196603 & 0.131604308 \\ \mathrm{C} & -2.787300448 & -1.233516111 & 0.103856176 \\ \mathrm{C} & -2.675584154 & -4.966940257 & 0.034646280 \\ \mathrm{C} & -4.966856852 & -2.668319860 & 0.261120679 \\ \mathrm{~N} & -1.408158418 & 1.409374542 & -0.024211861 \\ \mathrm{~N} & 1.434513793 & 1.423980569 & -0.010355947 \\ \mathrm{~N} & 1.411872190 & -1.421899082 & -0.008152732 \\ \mathrm{~N} & -1.427862636 & -1.416451457 & -0.003017646 \\ \mathrm{Fe} & 0.000000000 & 0.000000000 & 0.000000000 \\ \mathrm{C} & -0.000000000 & -0.000000000 & 1.890351664 \\ \mathrm{~N} & -0.003906497 & 0.002626710 & 3.064960961 \\ \mathrm{H} & -0.973917496 & -0.674364043 & -5.203411249 \\ \mathrm{H} & 1.232911015 & 0.609890159 & -4.974920645 \\ \mathrm{H} & -1.794641456 & -1.079678991 & -2.568151122 \\ & -1.843001408 & 1.037337232 & -2.522643560 \\ \mathrm{H} & -0.000895006 & 4.506829121 & -0.114855861\end{array}$




\begin{tabular}{|c|c|c|c|}
\hline $\mathrm{H}$ & 4.500804614 & -0.012563795 & 0.245861935 \\
\hline $\mathrm{H}$ & -0.024213846 & -4.505982774 & -0.112423434 \\
\hline & -3.707718151 & 5.244287002 & -0.082583184 \\
\hline & -2.159309261 & 5.405246627 & -0.932347689 \\
\hline & -2.204686613 & 5.430737161 & 0.839487756 \\
\hline & -5.337394873 & 2.233012717 & 1.096638109 \\
\hline & -5.465815326 & 2.185539134 & -0.670860076 \\
\hline & -5.222946385 & 3.733952380 & 0.160121517 \\
\hline & 2.772591674 & 5.408038846 & 0.987201303 \\
\hline & 1.845540593 & 5.470400078 & -0.522245401 \\
\hline & 3.609192022 & 5.240273173 & -0.564383066 \\
\hline & 5.510380213 & 1.772884735 & -0.116501034 \\
\hline & 5.178347272 & 4.631806668 & 0.988379974 \\
\hline & 6.737694865 & 3.700154124 & 0.607984 \\
\hline 11 & 5.393995492 & -1.840489722 & 0.821818428 \\
\hline $\mathrm{H}$ & 5.463056920 & -2.736988200 & -0.706993863 \\
\hline $\mathrm{H}$ & 5.164730268 & -3.604222634 & 0.809657029 \\
\hline $\mathrm{H}$ & 1.766865792 & -5.492009174 & 0.342604441 \\
\hline $\mathrm{H}$ & 4.616898640 & -5.171125346 & -0.789170789 \\
\hline $\mathrm{H}$ & 3.711684208 & -6.726787223 & -0.331660912 \\
\hline 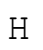 & -2.229198824 & -5.423479285 & 0.9327383 \\
\hline $\mathrm{H}$ & -2.186057443 & -5.425888567 & -0.839396 \\
\hline $\mathrm{H}$ & -3.734035823 & -5.258011669 & 0.0092669 \\
\hline $\mathrm{H}$ & -5.257508621 & -3.727070527 & 0.26753611 \\
\hline $\mathrm{H}$ & -5.507348046 & -2.182291441 & -0.56751252 \\
\hline & -5.338891071 & -2.219850219 & 1.196887 \\
\hline
\end{tabular}

Coordinates starting from the X-ray crystal structure and reducing the $\mathrm{Meso}_{\text {alpha }}-\mathrm{Fe}-\mathrm{Meso}_{\text {gamma }}$ angle His-heme-CN, $160.5^{\circ} \alpha$-meso-Fe- $\gamma$-meso angle

$\begin{array}{lrrr}\mathrm{C} & 0.508110 & -0.343336 & -4.196620 \\ \mathrm{~N} & 1.324282 & 0.731600 & -3.892087 \\ \mathrm{C} & -0.041687 & -0.735263 & -3.001339 \\ \mathrm{C} & 1.248850 & 0.956353 & -2.553662 \\ \mathrm{~N} & 0.426347 & 0.080753 & -1.991173 \\ \mathrm{H} & 0.398487 & -0.719814 & -5.206741 \\ \mathrm{H} & 1.885424 & 1.262585 & -4.549844 \\ \mathrm{H} & -0.733627 & -1.543586 & -2.793447 \\ \mathrm{H} & 1.787940 & 1.740561 & -2.033085 \\ \mathrm{C} & -2.444587 & -2.412618 & -0.038818 \\ \mathrm{C} & -2.293493 & 2.373334 & -0.745122 \\ \mathrm{C} & 2.136338 & 2.325922 & 1.193383 \\ \mathrm{C} & 2.381363 & -2.384457 & 0.114540 \\ \mathrm{C} & -2.832087 & -1.101888 & -0.285136 \\ \mathrm{C} & -4.183201 & -0.685790 & -0.601493 \\ \mathrm{C} & -4.119552 & 0.655768 & -0.903093 \\ \mathrm{C} & -2.736845 & 1.058496 & -0.719791 \\ \mathrm{C} & -5.230822 & 1.578136 & -1.288918 \\ \mathrm{C} & -5.375940 & -1.587127 & -0.591485 \\ \mathrm{C} & -1.056482 & 2.805562 & -0.270657 \\ \mathrm{C} & -0.722315 & 4.186178 & 0.003240 \\ \mathrm{C} & 0.499570 & 4.175065 & 0.657932 \\ \mathrm{C} & 0.914031 & 2.777295 & 0.715962\end{array}$




\begin{tabular}{|c|c|c|c|}
\hline $\mathrm{C}$ & -1.564900 & 5.361050 & -0.368546 \\
\hline $\mathrm{C}$ & 1.291133 & 5.279747 & 1.168146 \\
\hline $\mathrm{C}$ & 0.872790 & 6.522458 & 1.481834 \\
\hline $\mathrm{C}$ & 2.631776 & 1.033403 & 1.022996 \\
\hline $\mathrm{C}$ & 3.988911 & 0.628005 & 1.300892 \\
\hline $\mathrm{C}$ & 4.096031 & -0.692288 & 0.883390 \\
\hline $\mathrm{C}$ & 2.766283 & -1.080889 & 0.427319 \\
\hline C & 5.028125 & 1.482859 & 1.948403 \\
\hline C & 5.241350 & -1.583467 & 0.922259 \\
\hline C & 6.545326 & -1.242085 & 0.950140 \\
\hline C & 1.072171 & -2.823463 & -0.030738 \\
\hline C & 0.672249 & -4.224954 & -0.032363 \\
\hline $\mathrm{C}$ & -0.699971 & -4.237124 & 0.025904 \\
\hline $\mathrm{C}$ & -1.122664 & -2.845085 & 0.008467 \\
\hline $\mathrm{C}$ & 1.620076 & -5.380243 & -0.038115 \\
\hline $\mathrm{C}$ & -1.628855 & -5.406514 & 0.086663 \\
\hline $\mathrm{N}$ & -1.964360 & -0.028067 & -0.369162 \\
\hline $\mathrm{N}$ & -0.045349 & 1.967633 & 0.142693 \\
\hline $\mathrm{N}$ & 1.914499 & -0.009200 & 0.479763 \\
\hline $\mathrm{N}$ & -0.035617 & -2.010013 & -0.044091 \\
\hline $\mathrm{Fe}$ & -0.044671 & -0.013950 & 0.042873 \\
\hline $\mathrm{H}$ & -3.227635 & -3.169443 & 0.018521 \\
\hline $\mathrm{H}$ & -3.018373 & 3.136426 & -1.028287 \\
\hline $\mathrm{H}$ & 2.803471 & 3.057658 & 1.646961 \\
\hline $\mathrm{H}$ & 3.157358 & -3.149895 & 0.110760 \\
\hline $\mathrm{H}$ & -6.149419 & 1.023139 & -1.520336 \\
\hline $\mathrm{H}$ & -4.972738 & 2.177588 & -2.176291 \\
\hline $\mathrm{H}$ & -5.469581 & 2.286741 & -0.478580 \\
\hline $\mathrm{H}$ & -5.573980 & -1.981508 & 0.417863 \\
\hline $\mathrm{H}$ & -5.238526 & -2.454117 & -1.257051 \\
\hline $\mathrm{H}$ & -6.280509 & -1.058702 & -0.920393 \\
\hline $\mathrm{H}$ & -2.204588 & 5.690262 & 0.468163 \\
\hline $\mathrm{H}$ & -2.226941 & 5.133758 & -1.215933 \\
\hline $\mathrm{H}$ & -0.934911 & 6.218861 & -0.648850 \\
\hline $\mathrm{H}$ & 2.353966 & 5.064275 & 1.322877 \\
\hline $\mathrm{H}$ & -0.169848 & 6.830987 & 1.413485 \\
\hline $\mathrm{H}$ & 1.579452 & 7.266436 & 1.851728 \\
\hline $\mathrm{H}$ & 4.575897 & 2.253748 & 2.588167 \\
\hline $\mathrm{H}$ & 5.665785 & 1.999808 & 1.210813 \\
\hline $\mathrm{H}$ & 5.694236 & 0.871887 & 2.575805 \\
\hline $\mathrm{H}$ & 5.009003 & -2.654066 & 0.921838 \\
\hline $\mathrm{H}$ & 6.884735 & -0.207079 & 0.908776 \\
\hline $\mathrm{H}$ & 7.319123 & -2.009550 & 0.995065 \\
\hline $\mathrm{H}$ & 2.202671 & -5.431986 & 0.896658 \\
\hline $\mathrm{H}$ & 2.342512 & -5.312783 & -0.867479 \\
\hline $\mathrm{H}$ & 1.086698 & -6.334240 & -0.142328 \\
\hline $\mathrm{H}$ & -1.080438 & -6.356377 & 0.037607 \\
\hline $\mathrm{H}$ & -2.351601 & -5.400493 & -0.745938 \\
\hline $\mathrm{H}$ & -2.212357 & -5.411185 & 1.021573 \\
\hline C & -0.367586 & -0.186306 & 1.900166 \\
\hline $\mathrm{N}$ & -0.580346 & -0.286359 & 3.050616 \\
\hline
\end{tabular}


His-heme-CN, $159.5^{\circ} \alpha$-meso-Fe- $\gamma$-meso angle

\begin{tabular}{|c|c|c|c|}
\hline C & 0.507325 & -0.342699 & -4.207034 \\
\hline $\mathrm{N}$ & 1.329335 & 0.727658 & -3.902155 \\
\hline C & -0.043925 & -0.732584 & -3.011718 \\
\hline $\mathrm{C}$ & 1.255634 & 0.951966 & -2.563493 \\
\hline $\mathrm{N}$ & 0.428721 & 0.080390 & -2.001307 \\
\hline $\mathrm{H}$ & 0.395239 & -0.717899 & -5.217358 \\
\hline $\mathrm{H}$ & 1.893033 & 1.256031 & -4.559827 \\
\hline $\mathrm{H}$ & -0.740067 & -1.537301 & -2.803803 \\
\hline $\mathrm{H}$ & 1.799180 & 1.732833 & -2.042537 \\
\hline C & -2.442695 & -2.406757 & -0.019711 \\
\hline C & -2.287091 & 2.372814 & -0.765433 \\
\hline C & 2.126490 & 2.319018 & 1.209050 \\
\hline C & 2.384092 & -2.381661 & 0.093504 \\
\hline C & -2.830291 & -1.097903 & -0.276641 \\
\hline C & -4.181847 & -0.682138 & -0.591819 \\
\hline C & -4.116706 & 0.656137 & -0.908116 \\
\hline C & -2.732706 & 1.058528 & -0.733307 \\
\hline C & -5.227635 & 1.576542 & -1.299511 \\
\hline C & -5.376036 & -1.581338 & -0.569089 \\
\hline C & -1.051753 & 2.805136 & -0.285527 \\
\hline C & -0.719799 & 4.184762 & -0.004208 \\
\hline C & 0.494956 & 4.171330 & 0.664070 \\
\hline C & 0.909221 & 2.773428 & 0.721163 \\
\hline C & -1.558125 & 5.360974 & -0.381244 \\
\hline C & 1.281495 & 5.274232 & 1.185714 \\
\hline C & 0.861291 & 6.517026 & 1.496634 \\
\hline C & 2.626966 & 1.029314 & 1.028979 \\
\hline C & 3.983821 & 0.623890 & 1.308366 \\
\hline C & 4.096000 & -0.691593 & 0.876439 \\
\hline C & 2.768542 & -1.078973 & 0.412330 \\
\hline C & 5.018591 & 1.474324 & 1.968654 \\
\hline C & 5.243728 & -1.579800 & 0.908673 \\
\hline C & 6.546632 & -1.235167 & 0.946486 \\
\hline C & 1.074092 & -2.821658 & -0.045437 \\
\hline C & 0.673047 & -4.222906 & -0.038483 \\
\hline C & -0.698657 & -4.233858 & 0.035006 \\
\hline C & -1.120800 & -2.841622 & 0.016285 \\
\hline C & 1.619891 & -5.378964 & -0.050793 \\
\hline C & -1.627602 & -5.402450 & 0.109194 \\
\hline $\mathrm{N}$ & -1.961311 & -0.026386 & -0.375741 \\
\hline $\mathrm{N}$ & -0.044064 & 1.966435 & 0.134143 \\
\hline $\mathrm{N}$ & 1.915184 & -0.009155 & 0.471526 \\
\hline $\mathrm{N}$ & -0.033643 & -2.008307 & -0.051725 \\
\hline $\mathrm{Fe}$ & -0.042260 & -0.013283 & 0.031957 \\
\hline $\mathrm{H}$ & -3.226375 & -3.162063 & 0.048543 \\
\hline $\mathrm{H}$ & -3.010434 & 3.135804 & -1.052849 \\
\hline $\mathrm{H}$ & 2.788972 & 3.048355 & 1.673248 \\
\hline $\mathrm{H}$ & 3.160623 & -3.146569 & 0.085421 \\
\hline $\mathrm{H}$ & -6.147955 & 1.020834 & -1.52215 \\
\hline
\end{tabular}




\begin{tabular}{|c|c|c|c|}
\hline $\mathrm{H}$ & -4.971684 & 2.166518 & -2.193841 \\
\hline $\mathrm{H}$ & -5.462479 & 2.293622 & -0.495502 \\
\hline $\mathrm{H}$ & -5.570381 & -1.966723 & 0.444428 \\
\hline $\mathrm{H}$ & -5.242655 & -2.454230 & -1.227704 \\
\hline $\mathrm{H}$ & -6.281103 & -1.054353 & -0.898868 \\
\hline $\mathrm{H}$ & -2.205001 & 5.689049 & 0.450352 \\
\hline $\mathrm{H}$ & -2.212587 & 5.136054 & -1.235161 \\
\hline $\mathrm{H}$ & -0.924872 & 6.218784 & -0.654051 \\
\hline $\mathrm{H}$ & 2.342154 & 5.057232 & 1.352497 \\
\hline $\mathrm{H}$ & -0.179943 & 6.827455 & 1.416542 \\
\hline $\mathrm{H}$ & 1.564773 & 7.259248 & 1.876009 \\
\hline $\mathrm{H}$ & 4.562004 & 2.237232 & 2.614848 \\
\hline $\mathrm{H}$ & 5.657787 & 2.000514 & 1.238989 \\
\hline $\mathrm{H}$ & 5.683769 & 0.858324 & 2.592104 \\
\hline $\mathrm{H}$ & 5.014602 & -2.651012 & 0.892745 \\
\hline $\mathrm{H}$ & 6.883247 & -0.198754 & 0.920515 \\
\hline $\mathrm{H}$ & 7.322413 & -2.000999 & 0.984348 \\
\hline $\mathrm{H}$ & 2.213145 & -5.427852 & 0.877450 \\
\hline $\mathrm{H}$ & 2.332750 & -5.315302 & -0.888686 \\
\hline $\mathrm{H}$ & 1.084610 & -6.332905 & -0.145226 \\
\hline $\mathrm{H}$ & -1.080328 & -6.352865 & 0.057886 \\
\hline $\mathrm{H}$ & -2.358647 & -5.398787 & -0.716149 \\
\hline $\mathrm{H}$ & -2.201653 & -5.403421 & 1.049914 \\
\hline C & -0.364092 & -0.186084 & 1.889474 \\
\hline $\mathrm{N}$ & -0.576186 & -0.286444 & 3.039970 \\
\hline \multicolumn{4}{|c|}{ His-heme-CN, $158.4^{\circ} \alpha$-meso-Fe- $\gamma$-meso angle } \\
\hline $\mathrm{C}$ & 0.500520 & -0.343218 & -4.215636 \\
\hline $\mathrm{N}$ & 1.333106 & 0.719132 & -3.911521 \\
\hline $\mathrm{C}$ & -0.052931 & -0.728093 & -3.019727 \\
\hline $\mathrm{C}$ & 1.263763 & 0.943274 & -2.572578 \\
\hline $\mathrm{N}$ & 0.429313 & 0.079506 & -2.009490 \\
\hline $\mathrm{H}$ & 0.383368 & -0.716949 & -5.225922 \\
\hline $\mathrm{H}$ & 1.900987 & 1.242322 & -4.569727 \\
\hline $\mathrm{H}$ & -0.756702 & -1.525915 & -2.810931 \\
\hline $\mathrm{H}$ & 1.815928 & 1.718497 & -2.052237 \\
\hline $\mathrm{C}$ & -2.441433 & -2.400974 & 0.000891 \\
\hline $\mathrm{C}$ & -2.278549 & 2.371130 & -0.787114 \\
\hline C & 2.116758 & 2.312352 & 1.228209 \\
\hline $\mathrm{C}$ & 2.386107 & -2.376563 & 0.070946 \\
\hline $\mathrm{C}$ & -2.828529 & -1.093970 & -0.266258 \\
\hline C & -4.179941 & -0.677853 & -0.582617 \\
\hline C & -4.112139 & 0.656261 & -0.915352 \\
\hline C & -2.726828 & 1.057788 & -0.747546 \\
\hline C & -5.221684 & 1.575089 & -1.314384 \\
\hline $\mathrm{C}$ & -5.376260 & -1.573843 & -0.546758 \\
\hline $\mathrm{C}$ & -1.045542 & 2.803335 & -0.300292 \\
\hline C & -0.715991 & 4.182026 & -0.012630 \\
\hline C & 0.490264 & 4.166770 & 0.671417 \\
\hline C & 0.904343 & 2.769023 & 0.729973 \\
\hline $\mathrm{C}$ & -1.549323 & 5.359355 & -0.397189 \\
\hline $\mathrm{C}$ & 1.269880 & 5.268280 & 1.206091 \\
\hline
\end{tabular}




\begin{tabular}{|c|c|c|c|}
\hline C & 0.846525 & 6.511061 & 1.512813 \\
\hline C & 2.621693 & 1.025567 & 1.037377 \\
\hline C & 3.978661 & 0.619758 & 1.316309 \\
\hline C & 4.096135 & -0.689546 & 0.867204 \\
\hline $\mathrm{C}$ & 2.770342 & -1.075155 & 0.395769 \\
\hline C & 5.008834 & 1.464756 & 1.990739 \\
\hline $\mathrm{C}$ & 5.246586 & -1.574360 & 0.888493 \\
\hline C & 6.548394 & -1.227283 & 0.941156 \\
\hline C & 1.075431 & -2.817759 & -0.060249 \\
\hline C & 0.674191 & -4.218822 & -0.045590 \\
\hline C & -0.696735 & -4.229309 & 0.044733 \\
\hline $\mathrm{C}$ & -1.119302 & -2.837255 & 0.026197 \\
\hline C & 1.620460 & -5.375276 & -0.065248 \\
\hline $\mathrm{C}$ & -1.624793 & -5.397704 & 0.133211 \\
\hline $\mathrm{N}$ & -1.957387 & -0.025314 & -0.380535 \\
\hline $\mathrm{N}$ & -0.042175 & 1.964016 & 0.128458 \\
\hline N & 1.915071 & -0.007504 & 0.464002 \\
\hline $\mathrm{N}$ & -0.032730 & -2.004934 & -0.057625 \\
\hline $\mathrm{Fe}$ & -0.040593 & -0.011818 & 0.023441 \\
\hline $\mathrm{H}$ & -3.225475 & -3.154652 & 0.082054 \\
\hline $\mathrm{H}$ & -2.999443 & 3.134206 & -1.080444 \\
\hline $\mathrm{H}$ & 2.773226 & 3.038548 & 1.705729 \\
\hline $\mathrm{H}$ & 3.163145 & -3.140966 & 0.057791 \\
\hline $\mathrm{H}$ & -6.143864 & 1.019379 & -1.529164 \\
\hline $\mathrm{H}$ & -4.966146 & 2.154725 & -2.215577 \\
\hline $\mathrm{H}$ & -5.453063 & 2.301223 & -0.517535 \\
\hline $\mathrm{H}$ & -5.566935 & -1.950066 & 0.470857 \\
\hline $\mathrm{H}$ & -5.247690 & -2.452689 & -1.198396 \\
\hline $\mathrm{H}$ & -6.281500 & -1.047656 & -0.877263 \\
\hline $\mathrm{H}$ & -2.205119 & 5.686577 & 0.427722 \\
\hline $\mathrm{H}$ & -2.194328 & 5.136194 & -1.258756 \\
\hline $\mathrm{H}$ & -0.912403 & 6.217075 & -0.661500 \\
\hline $\mathrm{H}$ & 2.327711 & 5.049878 & 1.388063 \\
\hline $\mathrm{H}$ & -0.192916 & 6.823339 & 1.418037 \\
\hline $\mathrm{H}$ & 1.545469 & 7.251686 & 1.903577 \\
\hline $\mathrm{H}$ & 4.547657 & 2.219483 & 2.643207 \\
\hline $\mathrm{H}$ & 5.650720 & 1.999820 & 1.269950 \\
\hline $\mathrm{H}$ & 5.671967 & 0.843076 & 2.610717 \\
\hline $\mathrm{H}$ & 5.020884 & -2.645685 & 0.848432 \\
\hline $\mathrm{H}$ & 6.882554 & -0.189781 & 0.939475 \\
\hline $\mathrm{H}$ & 7.325894 & -1.991842 & 0.967208 \\
\hline $\mathrm{H}$ & 2.225102 & -5.420867 & 0.855843 \\
\hline $\mathrm{H}$ & 2.322920 & -5.315209 & -0.912118 \\
\hline $\mathrm{H}$ & 1.083819 & -6.329421 & -0.149201 \\
\hline $\mathrm{H}$ & -1.078111 & -6.348311 & 0.079245 \\
\hline $\mathrm{H}$ & -2.364692 & -5.396799 & -0.684228 \\
\hline $\mathrm{H}$ & -2.188671 & -5.395196 & 1.080018 \\
\hline C & -0.358934 & -0.187405 & 1.881256 \\
\hline $\mathrm{N}$ & -0.568902 & -0.289517 & 3.031973 \\
\hline \multicolumn{4}{|c|}{ is-heme- $\mathrm{CN}, 157.4^{\circ} \alpha$-meso- $\mathrm{Fe}-\gamma$-meso angle } \\
\hline $\mathrm{C}$ & 0.500565 & -0.342870 & -4.226239 \\
\hline
\end{tabular}




\begin{tabular}{|c|c|c|c|}
\hline $\mathrm{N}$ & 1.338179 & 0.715427 & -3.921860 \\
\hline $\mathrm{C}$ & -0.053674 & -0.726320 & -3.030212 \\
\hline $\mathrm{C}$ & 1.269898 & 0.939570 & -2.582805 \\
\hline $\mathrm{N}$ & 0.431905 & 0.079150 & -2.019915 \\
\hline $\mathrm{H}$ & 0.381357 & -0.715522 & -5.236680 \\
\hline $\mathrm{H}$ & 1.908289 & 1.236253 & -4.580009 \\
\hline $\mathrm{H}$ & -0.760656 & -1.521282 & -2.821290 \\
\hline $\mathrm{H}$ & 1.825506 & 1.712132 & -2.062181 \\
\hline C & -2.438769 & -2.394247 & 0.019470 \\
\hline $\mathrm{C}$ & -2.272334 & 2.370798 & -0.807023 \\
\hline $\mathrm{C}$ & 2.106545 & 2.304882 & 1.242362 \\
\hline $\mathrm{C}$ & 2.388688 & -2.373960 & 0.050708 \\
\hline $\mathrm{C}$ & -2.826243 & -1.089366 & -0.258477 \\
\hline $\mathrm{C}$ & -4.178153 & -0.673735 & -0.573522 \\
\hline $\mathrm{C}$ & -4.109206 & 0.656795 & -0.920735 \\
\hline C & -2.722662 & 1.057970 & -0.761486 \\
\hline C & -5.218704 & 1.573457 & -1.324768 \\
\hline C & -5.375900 & -1.567195 & -0.524349 \\
\hline $\mathrm{C}$ & -1.040765 & 2.803025 & -0.315432 \\
\hline $\mathrm{C}$ & -0.713412 & 4.180684 & -0.020147 \\
\hline $\mathrm{C}$ & 0.485655 & 4.162788 & 0.676680 \\
\hline $\mathrm{C}$ & 0.899421 & 2.764860 & 0.733892 \\
\hline $\mathrm{C}$ & -1.542685 & 5.359430 & -0.409006 \\
\hline $\mathrm{C}$ & 1.259869 & 5.262352 & 1.223024 \\
\hline C & 0.833645 & 6.504141 & 1.529748 \\
\hline C & 2.616938 & 1.021204 & 1.042197 \\
\hline $\mathrm{C}$ & 3.973708 & 0.615550 & 1.322583 \\
\hline $\mathrm{C}$ & 4.096212 & -0.688809 & 0.859928 \\
\hline $\mathrm{C}$ & 2.772643 & -1.073293 & 0.380822 \\
\hline $\mathrm{C}$ & 4.999594 & 1.456080 & 2.008974 \\
\hline $\mathrm{C}$ & 5.248890 & -1.570721 & 0.875371 \\
\hline $\mathrm{C}$ & 6.549611 & -1.221191 & 0.938545 \\
\hline $\mathrm{C}$ & 1.077297 & -2.816061 & -0.074965 \\
\hline $\mathrm{C}$ & 0.674876 & -4.216843 & -0.051748 \\
\hline $\mathrm{C}$ & -0.695165 & -4.225780 & 0.053418 \\
\hline $\mathrm{C}$ & -1.116986 & -2.833411 & 0.033452 \\
\hline C & 1.619895 & -5.374237 & -0.076839 \\
\hline $\mathrm{C}$ & -1.623073 & -5.393170 & 0.155938 \\
\hline $\mathrm{N}$ & -1.954245 & -0.023330 & -0.387633 \\
\hline $\mathrm{N}$ & -0.040899 & 1.962850 & 0.119186 \\
\hline $\mathrm{N}$ & 1.915864 & -0.007654 & 0.455604 \\
\hline $\mathrm{N}$ & -0.030580 & -2.003246 & -0.065418 \\
\hline $\mathrm{Fe}$ & -0.038010 & -0.011127 & 0.011813 \\
\hline $\mathrm{H}$ & -3.223342 & -3.146114 & 0.111906 \\
\hline $\mathrm{H}$ & -2.991872 & 3.133823 & -1.103849 \\
\hline $\mathrm{H}$ & 2.757964 & 3.028554 & 1.730614 \\
\hline $\mathrm{H}$ & 3.166125 & -3.137919 & 0.033824 \\
\hline $\mathrm{H}$ & -6.142561 & 1.017159 & -1.530550 \\
\hline $\mathrm{H}$ & -4.965695 & 2.143615 & -2.232705 \\
\hline $\mathrm{H}$ & -5.445995 & 2.307841 & -0.534323 \\
\hline $\mathrm{H}$ & -5.562952 & -1.933621 & 0.497496 \\
\hline $\mathrm{H}$ & -5.251516 & -2.452277 & -1.168278 \\
\hline
\end{tabular}




\begin{tabular}{|c|c|c|c|}
\hline $\mathrm{H}$ & & & \\
\hline & -6.281579 & -1.042549 & -0.856038 \\
\hline $\mathrm{H}$ & -2.207062 & 5.683672 & 0.410167 \\
\hline $\mathrm{H}$ & -2.178551 & 5.139561 & -1.278235 \\
\hline $\mathrm{H}$ & -0.902913 & 6.217989 & -0.663353 \\
\hline $\mathrm{H}$ & 2.315789 & 5.043199 & 1.414797 \\
\hline $\mathrm{H}$ & -0.204748 & 6.816989 & 1.425703 \\
\hline $\mathrm{H}$ & 1.528815 & 7.243309 & 1.929895 \\
\hline $\mathrm{H}$ & 4.534414 & 2.203854 & 2.666561 \\
\hline $\mathrm{H}$ & 5.643345 & 1.998880 & 1.295696 \\
\hline $\mathrm{H}$ & 5.661381 & 0.829907 & 2.625846 \\
\hline $\mathrm{H}$ & 5.026231 & -2.642031 & 0.820019 \\
\hline $\mathrm{H}$ & 6.881195 & -0.182971 & 0.952324 \\
\hline $\mathrm{H}$ & 7.328848 & -1.984167 & 0.958089 \\
\hline $\mathrm{H}$ & 2.234000 & -5.417078 & 0.838140 \\
\hline $\mathrm{H}$ & 2.313592 & -5.318046 & -0.931145 \\
\hline $\mathrm{H}$ & 1.081554 & -6.328179 & -0.151633 \\
\hline $\mathrm{H}$ & -1.077611 & -6.344393 & 0.100224 \\
\hline $\mathrm{H}$ & -2.371204 & -5.394975 & -0.653970 \\
\hline $\mathrm{H}$ & -2.177316 & -5.386489 & 1.108365 \\
\hline $\mathrm{C}$ & -0.355845 & -0.186290 & 1.869972 \\
\hline $\mathrm{N}$ & -0.565452 & -0.288254 & 3.020708 \\
\hline His $-\mathrm{H}$ & $\mathrm{N}, 156.4^{\circ} \alpha-\mathrm{me}$ & $\mathrm{e}-\gamma$-meso angle & \\
\hline $\mathrm{C}$ & 0.498427 & -0.343470 & -4.235785 \\
\hline $\mathrm{N}$ & 1.341626 & 0.710502 & -3.931922 \\
\hline $\mathrm{C}$ & -0.057655 & -0.723534 & -3.039542 \\
\hline C & 1.275820 & 0.934544 & -2.592711 \\
\hline $\mathrm{N}$ & 0.433460 & 0.078708 & -2.029303 \\
\hline $\mathrm{H}$ & 0.376654 & -0.715512 & -5.246140 \\
\hline $\mathrm{H}$ & 1.914082 & 1.228337 & -4.590386 \\
\hline $\mathrm{H}$ & -0.769150 & -1.514314 & -2.830099 \\
\hline $\mathrm{H}$ & 1.836231 & 1.703824 & -2.072392 \\
\hline $\mathrm{C}$ & -2.436553 & -2.387777 & 0.038691 \\
\hline $\mathrm{C}$ & -2.264416 & 2.369504 & -0.828258 \\
\hline $\mathrm{C}$ & 2.096824 & 2.297597 & 1.258195 \\
\hline $\mathrm{C}$ & 2.390706 & -2.369873 & 0.028972 \\
\hline $\mathrm{C}$ & -2.823917 & -1.085030 & -0.249837 \\
\hline C & -4.175948 & -0.669139 & -0.564857 \\
\hline C & -4.104955 & 0.657205 & -0.927636 \\
\hline $\mathrm{C}$ & -2.717158 & 1.057516 & -0.776228 \\
\hline $\mathrm{C}$ & -5.213636 & 1.572087 & -1.337967 \\
\hline $\mathrm{C}$ & -5.375725 & -1.559039 & -0.501952 \\
\hline $\mathrm{C}$ & -1.034965 & 2.801724 & -0.330542 \\
\hline C & -0.710185 & 4.178352 & -0.028035 \\
\hline C & 0.480815 & 4.158198 & 0.682877 \\
\hline $\mathrm{C}$ & 0.894715 & 2.760343 & 0.739938 \\
\hline $\mathrm{C}$ & -1.535058 & 5.358336 & -0.422507 \\
\hline $\mathrm{C}$ & 1.248361 & 5.256113 & 1.241766 \\
\hline C & 0.818208 & 6.496600 & 1.548225 \\
\hline C & 2.611966 & 1.017001 & 1.048038 \\
\hline $\mathrm{C}$ & 3.968614 & 0.610932 & 1.328822 \\
\hline . & 4.096164 & -0.687606 & 0.850981 \\
\hline
\end{tabular}




\begin{tabular}{|c|c|c|c|}
\hline $\mathrm{C}$ & 2.774551 & -1.070330 & 0.364364 \\
\hline $\mathrm{C}$ & 4.990126 & 1.446186 & 2.028123 \\
\hline $\mathrm{C}$ & 5.251183 & -1.566401 & 0.857820 \\
\hline $\mathrm{C}$ & 6.550789 & -1.215309 & 0.934852 \\
\hline $\mathrm{C}$ & 1.078789 & -2.813029 & -0.089899 \\
\hline $\mathrm{C}$ & 0.675850 & -4.213489 & -0.057957 \\
\hline C & -0.693064 & -4.221350 & 0.063186 \\
\hline $\mathrm{C}$ & -1.114959 & -2.828991 & 0.042098 \\
\hline $\mathrm{C}$ & 1.619919 & -5.371548 & -0.088952 \\
\hline $\mathrm{C}$ & -1.620023 & -5.388131 & 0.180833 \\
\hline $\mathrm{N}$ & -1.950259 & -0.022068 & -0.394419 \\
\hline $\mathrm{N}$ & -0.038855 & 1.960894 & 0.111165 \\
\hline $\mathrm{N}$ & 1.916156 & -0.006655 & 0.446627 \\
\hline $\mathrm{N}$ & -0.029159 & -2.000398 & -0.072853 \\
\hline $\mathrm{Fe}$ & -0.035764 & -0.010098 & 0.001255 \\
\hline $\mathrm{H}$ & -3.221421 & -3.137653 & 0.144271 \\
\hline $\mathrm{H}$ & -2.982028 & 3.132619 & -1.129626 \\
\hline $\mathrm{H}$ & 2.742219 & 3.018009 & 1.759237 \\
\hline $\mathrm{H}$ & 3.168556 & -3.133422 & 0.008180 \\
\hline $\mathrm{H}$ & -6.139579 & 1.015918 & -1.534507 \\
\hline $\mathrm{H}$ & -4.962376 & 2.131530 & -2.253024 \\
\hline $\mathrm{H}$ & -5.436439 & 2.315540 & -0.554771 \\
\hline $\mathrm{H}$ & -5.559212 & -1.915205 & 0.524141 \\
\hline $\mathrm{H}$ & -5.256203 & -2.450575 & -1.137848 \\
\hline $\mathrm{H}$ & -6.281512 & -1.035441 & -0.834938 \\
\hline $\mathrm{H}$ & -2.209708 & 5.679074 & 0.389602 \\
\hline $\mathrm{H}$ & -2.159840 & 5.141714 & -1.300592 \\
\hline $\mathrm{H}$ & -0.892372 & 6.218013 & -0.665256 \\
\hline $\mathrm{H}$ & 2.302110 & 5.036499 & 1.444536 \\
\hline $\mathrm{H}$ & -0.219055 & 6.809647 & 1.433818 \\
\hline $\mathrm{H}$ & 1.508694 & 7.234545 & 1.958645 \\
\hline $\mathrm{H}$ & 4.520683 & 2.186713 & 2.690824 \\
\hline $\mathrm{H}$ & 5.636478 & 1.996597 & 1.323091 \\
\hline $\mathrm{H}$ & 5.649799 & 0.814959 & 2.642096 \\
\hline $\mathrm{H}$ & 5.031562 & -2.637059 & 0.781426 \\
\hline $\mathrm{H}$ & 6.880042 & -0.176883 & 0.970127 \\
\hline $\mathrm{H}$ & 7.331526 & -1.976921 & 0.944669 \\
\hline $\mathrm{H}$ & 2.244264 & -5.410999 & 0.819277 \\
\hline $\mathrm{H}$ & 2.303959 & -5.319334 & -0.951225 \\
\hline $\mathrm{H}$ & 1.080314 & -6.325514 & -0.153594 \\
\hline $\mathrm{H}$ & -1.075321 & -6.339706 & 0.123576 \\
\hline $\mathrm{H}$ & -2.376753 & -5.393366 & -0.621031 \\
\hline $\mathrm{H}$ & -2.164092 & -5.376919 & 1.139032 \\
\hline $\mathrm{C}$ & -0.352156 & -0.186083 & 1.859809 \\
\hline $\mathrm{N}$ & -0.560800 & -0.288679 & 3.010652 \\
\hline \multicolumn{4}{|c|}{ His-heme-CN, $155.4^{\circ} \alpha$-meso-Fe- $\gamma$-meso angle } \\
\hline $\mathrm{C}$ & 0.498464 & -0.342970 & -4.247247 \\
\hline $\mathrm{N}$ & 1.346484 & 0.707057 & -3.943183 \\
\hline C & -0.058178 & -0.721748 & -3.050838 \\
\hline $\mathrm{C}$ & 1.281666 & 0.931173 & -2.603886 \\
\hline $\mathrm{N}$ & 0.436042 & 0.078526 & -2.040567 \\
\hline
\end{tabular}




\begin{tabular}{|c|c|c|c|}
\hline $\mathrm{H}$ & 0.374664 & -0.713979 & -5.25773 \\
\hline $\mathrm{H}$ & 1.921022 & 1.222621 & -4.60160 \\
\hline $\mathrm{H}$ & -0.772604 & -1.509855 & -2.84122 \\
\hline $\mathrm{H}$ & 1.845299 & 1.697972 & -2.08338 \\
\hline C & -2.433433 & -2.380723 & 0.05627 \\
\hline C & -2.258041 & 2.368980 & -0.84797 \\
\hline C & 2.086665 & 2.289822 & 1.27124 \\
\hline C & 2.393078 & -2.367088 & 0.00872 \\
\hline C & -2.821198 & -1.080275 & -0.24292 \\
\hline C & -4.173730 & -0.664674 & -0.55635 \\
\hline C & -4.101703 & 0.657784 & -0.93331 \\
\hline C & -2.712692 & 1.057497 & -0.7904 \\
\hline C & -5.210405 & 1.570548 & -1.34825 \\
\hline C & -5.374959 & -1.551544 & -0.47972 \\
\hline C & -1.030071 & 2.801168 & -0.34542 \\
\hline C & -0.707780 & 4.176697 & -0.03507 \\
\hline C & 0.475987 & 4.153789 & 0.68800 \\
\hline C & 0.889824 & 2.755853 & 0.7433 \\
\hline C & -1.528765 & 5.358158 & -0.4331 \\
\hline $\mathrm{C}$ & 1.237866 & 5.249620 & 1.2586 \\
\hline C & 0.804485 & 6.488693 & 1.5661 \\
\hline $\mathrm{C}$ & 2.607149 & 1.012506 & 1.05184 \\
\hline C & 3.963484 & 0.606433 & 1.3343 \\
\hline C & 4.096093 & -0.686910 & 0.8433 \\
\hline C & 2.776736 & -1.068256 & 0.34895 \\
\hline C & 4.980574 & 1.437031 & 2.0455 \\
\hline C & 5.253265 & -1.562733 & 0.84442 \\
\hline C & 6.551695 & -1.209980 & 0.9335 \\
\hline C & 1.080477 & -2.811074 & -0.1046 \\
\hline C & 0.676587 & -4.211161 & -0.06364 \\
\hline C & -0.691118 & -4.217339 & 0.0720 \\
\hline C & -1.112362 & -2.824744 & 0.0488 \\
\hline C & 1.619316 & -5.370213 & -0.0992 \\
\hline C & -1.617503 & -5.383014 & 0.20468 \\
\hline $\mathrm{N}$ & -1.946689 & -0.020275 & -0.40226 \\
\hline $\mathrm{N}$ & -0.037341 & 1.959445 & 0.1015 \\
\hline $\mathrm{N}$ & 1.916844 & -0.006601 & 0.4373 \\
\hline $\mathrm{N}$ & -0.027069 & -1.998339 & -0.0812 \\
\hline $\mathrm{Fe}$ & -0.033059 & -0.009351 & -0.0112 \\
\hline $\mathrm{H}$ & -3.218577 & -3.128568 & 0.17350 \\
\hline $\mathrm{H}$ & -2.974344 & 3.132094 & -1.1524 \\
\hline $\mathrm{H}$ & 2.726683 & 3.007376 & 1.7832 \\
\hline $\mathrm{H}$ & 3.171202 & -3.130269 & -0.01533 \\
\hline $\mathrm{H}$ & -6.138240 & 1.014193 & -1.53503 \\
\hline $\mathrm{H}$ & -4.962158 & 2.119967 & $-2.2701 \varepsilon$ \\
\hline $\mathrm{H}$ & -5.428440 & 2.322421 & $-0.5717 \varepsilon$ \\
\hline $\mathrm{H}$ & -5.554318 & -1.897915 & 0.5504 \\
\hline $\mathrm{H}$ & -5.260165 & -2.449125 & -1.1079 \\
\hline $\mathrm{H}$ & -6.281238 & -1.029237 & -0.8133 \\
\hline $\mathrm{H}$ & -2.212096 & 5.675267 & 0.3730 \\
\hline $\mathrm{H}$ & -2.143982 & 5.145231 & -1.3189 \\
\hline $\mathrm{H}$ & -0.883678 & 6.218915 & -0.6652 \\
\hline
\end{tabular}




\begin{tabular}{|c|c|c|c|}
\hline & & & \\
\hline $\mathrm{H}$ & 2.289732 & 5.029435 & 1.470254 \\
\hline $\mathrm{H}$ & -0.231768 & 6.801984 & 1.443516 \\
\hline $\mathrm{H}$ & 1.490948 & 7.225139 & 1.985953 \\
\hline $\mathrm{H}$ & 4.507015 & 2.171117 & 2.712401 \\
\hline $\mathrm{H}$ & 5.629370 & 1.994248 & 1.348166 \\
\hline $\mathrm{H}$ & 5.638254 & 0.801495 & 2.657168 \\
\hline $\mathrm{H}$ & 5.036692 & -2.632705 & 0.751307 \\
\hline $\mathrm{H}$ & 6.878475 & -0.171528 & 0.985920 \\
\hline $\mathrm{H}$ & 7.333995 & -1.970037 & 0.936483 \\
\hline $\mathrm{H}$ & 2.253005 & -5.406240 & 0.802707 \\
\hline $\mathrm{H}$ & 2.294419 & -5.322550 & -0.968731 \\
\hline $\mathrm{H}$ & 1.078242 & -6.323964 & -0.153906 \\
\hline $\mathrm{H}$ & -1.073866 & -6.335171 & 0.146791 \\
\hline $\mathrm{H}$ & -2.382204 & -5.391867 & -0.589540 \\
\hline $\mathrm{H}$ & -2.151964 & -5.366691 & 1.168171 \\
\hline C & -0.348930 & -0.184991 & 1.847714 \\
\hline $\mathrm{N}$ & -0.557158 & -0.287521 & 2.998572 \\
\hline His $-\mathrm{r}$ & $\mathrm{N}, 154.4^{\circ} \alpha-\mathrm{me}$ & e- $\gamma$-meso angle & \\
\hline $\mathrm{C}$ & 0.497811 & -0.342812 & -4.257989 \\
\hline $\mathrm{N}$ & 1.349893 & 0.703956 & -3.954149 \\
\hline C & -0.060599 & -0.718905 & -3.061563 \\
\hline $\mathrm{C}$ & 1.286938 & 0.927729 & -2.614686 \\
\hline $\mathrm{N}$ & 0.437902 & 0.078590 & -2.051146 \\
\hline $\mathrm{H}$ & 0.372229 & -0.713295 & -5.268442 \\
\hline $\mathrm{H}$ & 1.926243 & 1.217325 & -4.612695 \\
\hline $\mathrm{H}$ & -0.778599 & -1.503690 & -2.851730 \\
\hline $\mathrm{H}$ & 1.854213 & 1.691867 & -2.094241 \\
\hline $\mathrm{C}$ & -2.430543 & -2.373938 & 0.074344 \\
\hline $\mathrm{C}$ & -2.250579 & 2.367677 & -0.868378 \\
\hline C & 2.076916 & 2.282142 & 1.285228 \\
\hline C & 2.395021 & -2.363267 & -0.012638 \\
\hline C & -2.818422 & -1.075824 & -0.235369 \\
\hline C & -4.171221 & -0.659927 & -0.548007 \\
\hline $\mathrm{C}$ & -4.097580 & 0.658236 & -0.939798 \\
\hline C & -2.707355 & 1.056962 & -0.805137 \\
\hline C & -5.205782 & 1.569155 & -1.360156 \\
\hline C & -5.374337 & -1.542915 & -0.457286 \\
\hline C & -1.024596 & 2.799878 & -0.360209 \\
\hline C & -0.705055 & 4.174300 & -0.042385 \\
\hline C & 0.470969 & 4.148951 & 0.693541 \\
\hline $\mathrm{C}$ & 0.885150 & 2.751113 & 0.748009 \\
\hline C & -1.522070 & 5.357068 & -0.444817 \\
\hline C & 1.226309 & 5.242960 & 1.276192 \\
\hline C & 0.789053 & 6.480575 & 1.584105 \\
\hline $\mathrm{C}$ & 2.602197 & 1.008053 & 1.056315 \\
\hline $\mathrm{C}$ & 3.958219 & 0.601438 & 1.339816 \\
\hline $\mathrm{C}$ & 4.095810 & -0.686119 & 0.834742 \\
\hline C & 2.778585 & -1.065513 & 0.332529 \\
\hline $\mathrm{C}$ & 4.970884 & 1.426690 & 2.063477 \\
\hline C & 5.255036 & -1.559018 & 0.828417 \\
\hline $\mathrm{C}$ & 6.552283 & -1.205810 & 0.932461 \\
\hline
\end{tabular}




\begin{tabular}{|c|c|c|c|}
\hline C & 1.081977 & -2.808181 & -0.119781 \\
\hline $\mathrm{C}$ & 0.677696 & -4.207862 & -0.069617 \\
\hline $\mathrm{C}$ & -0.688575 & -4.212736 & 0.08156 \\
\hline $\mathrm{C}$ & -1.109898 & -2.820166 & 0.05650 \\
\hline C & 1.619356 & -5.367658 & -0.11032 \\
\hline $\mathrm{C}$ & -1.613592 & -5.377633 & 0.23007 \\
\hline $\mathrm{N}$ & -1.942540 & -0.019141 & -0.41002 \\
\hline $\mathrm{N}$ & -0.035237 & 1.957451 & 0.09272 \\
\hline $\mathrm{N}$ & 1.917192 & -0.005742 & 0.42750 \\
\hline $\mathrm{N}$ & -0.025488 & -1.995501 & -0.08962 \\
\hline $\mathrm{Fe}$ & -0.030587 & -0.008407 & -0.02304 \\
\hline $\mathrm{H}$ & -3.215752 & -3.119550 & 0.20483 \\
\hline $\mathrm{H}$ & -2.965310 & 3.130880 & -1.17643 \\
\hline $\mathrm{H}$ & 2.710862 & 2.996271 & 1.80953 \\
\hline $\mathrm{H}$ & 3.173442 & -3.126139 & -0.0399 \\
\hline $\mathrm{H}$ & -6.136097 & 1.013333 & -1.5358 \\
\hline $\mathrm{H}$ & -4.960541 & 2.106802 & $-2.2897 \varepsilon$ \\
\hline $\mathrm{H}$ & -5.417938 & 2.330683 & -0.5915 \\
\hline $\mathrm{H}$ & -5.549311 & -1.879422 & 0.57688 \\
\hline $\mathrm{H}$ & -5.265039 & -2.446517 & $-1.0777^{\circ}$ \\
\hline $\mathrm{H}$ & -6.280926 & -1.021364 & -0.7912 \\
\hline $\mathrm{H}$ & -2.214217 & 5.670880 & 0.3551 \\
\hline $\mathrm{H}$ & -2.127388 & 5.147411 & -1.3381 \\
\hline $\mathrm{H}$ & -0.874679 & 6.218821 & -0.6663 \\
\hline $\mathrm{H}$ & 2.276016 & 5.022327 & 1.49777 \\
\hline $\mathrm{H}$ & -0.246035 & 6.793949 & 1.45200 \\
\hline $\mathrm{H}$ & 1.470829 & 7.215756 & 2.0136 \\
\hline $\mathrm{H}$ & 4.493103 & 2.154773 & 2.73387 \\
\hline $\mathrm{H}$ & 5.623158 & 1.989910 & 1.37425 \\
\hline $\mathrm{H}$ & 5.625497 & 0.786513 & $2.6735^{\circ}$ \\
\hline $\mathrm{H}$ & 5.041303 & -2.627535 & 0.7143 \\
\hline $\mathrm{H}$ & 6.876986 & -0.168071 & 1.00664 \\
\hline $\mathrm{H}$ & 7.335759 & -1.964627 & 0.9264 \\
\hline $\mathrm{H}$ & 2.263480 & -5.399538 & 0.7843 \\
\hline $\mathrm{H}$ & 2.284311 & -5.324771 & -0.9878 \\
\hline $\mathrm{H}$ & 1.077221 & -6.321383 & -0.15382 \\
\hline $\mathrm{H}$ & -1.070494 & -6.330103 & 0.1721 \\
\hline $\mathrm{H}$ & -2.386400 & -5.391048 & $-0.5561 \varepsilon$ \\
\hline $\mathrm{H}$ & -2.138183 & -5.355694 & 1.1988 \\
\hline $\mathrm{C}$ & -0.345664 & -0.184332 & 1.8362 \\
\hline $\mathrm{N}$ & -0.553254 & -0.287244 & 2.9872 \\
\hline \multicolumn{4}{|c|}{ His-heme-CN, $153.4^{\circ} \alpha$-meso-Fe- $\gamma$-meso angle } \\
\hline $\mathrm{C}$ & 0.498636 & -0.341316 & -4.2704 \\
\hline $\mathrm{N}$ & 1.354832 & 0.701912 & -3.96605 \\
\hline $\mathrm{C}$ & -0.060162 & -0.716818 & -3.07399 \\
\hline $\mathrm{C}$ & 1.292473 & 0.925471 & -2.62647 \\
\hline $\mathrm{N}$ & 0.440689 & 0.078849 & -2.06330 \\
\hline $\mathrm{H}$ & 0.371393 & -0.710592 & -5.28113 \\
\hline $\mathrm{H}$ & 1.933002 & 1.213519 & -4.62436 \\
\hline $\mathrm{H}$ & -0.780529 & -1.499480 & -2.8642 \\
\hline $\mathrm{H}$ & 1.862327 & 1.687397 & -2.1056 \\
\hline
\end{tabular}




\begin{tabular}{|c|c|c|c|}
\hline C & -2.426723 & -2.366571 & 0.090930 \\
\hline C & -2.244555 & 2.367126 & -0.887583 \\
\hline C & 2.066624 & 2.273925 & 1.296845 \\
\hline C & 2.397391 & -2.360637 & -0.032854 \\
\hline C & -2.815290 & -1.070958 & -0.229303 \\
\hline C & -4.168726 & -0.655289 & -0.539674 \\
\hline $\mathrm{C}$ & -4.094442 & 0.658841 & -0.945135 \\
\hline C & -2.703037 & 1.056862 & -0.819405 \\
\hline C & -5.202953 & 1.567599 & -1.369329 \\
\hline $\mathrm{C}$ & -5.373157 & -1.534898 & -0.434770 \\
\hline C & -1.019938 & 2.799327 & -0.374952 \\
\hline $\mathrm{C}$ & -0.703009 & 4.172565 & -0.049126 \\
\hline C & 0.465967 & 4.144296 & 0.698089 \\
\hline C & 0.880217 & 2.746396 & 0.750395 \\
\hline C & -1.516445 & 5.356874 & -0.454253 \\
\hline $\mathrm{C}$ & 1.215634 & 5.236046 & 1.292180 \\
\hline $\mathrm{C}$ & 0.775338 & 6.472320 & 1.601108 \\
\hline $\mathrm{C}$ & 2.597274 & 1.003253 & 1.059135 \\
\hline $\mathrm{C}$ & 3.952813 & 0.596502 & 1.344899 \\
\hline $\mathrm{C}$ & 4.095526 & -0.685766 & 0.827367 \\
\hline $\mathrm{C}$ & 2.780756 & -1.063577 & 0.316970 \\
\hline $\mathrm{C}$ & 4.960853 & 1.416909 & 2.080426 \\
\hline $\mathrm{C}$ & 5.256720 & -1.555825 & 0.816154 \\
\hline $\mathrm{C}$ & 6.552683 & -1.201805 & 0.933196 \\
\hline $\mathrm{C}$ & 1.083745 & -2.806333 & -0.134952 \\
\hline $\mathrm{C}$ & 0.678653 & -4.205549 & -0.075326 \\
\hline C & -0.686112 & -4.208531 & 0.090157 \\
\hline $\mathrm{C}$ & -1.106837 & -2.815757 & 0.062374 \\
\hline C & 1.618880 & -5.366395 & -0.119907 \\
\hline C & -1.610128 & -5.372141 & 0.254449 \\
\hline $\mathrm{N}$ & -1.938834 & -0.017463 & -0.418736 \\
\hline $\mathrm{N}$ & -0.033713 & 1.955975 & 0.082509 \\
\hline $\mathrm{N}$ & 1.917918 & -0.005799 & 0.417409 \\
\hline $\mathrm{N}$ & -0.023215 & -1.993457 & -0.098971 \\
\hline $\mathrm{Fe}$ & -0.027735 & -0.007760 & -0.036482 \\
\hline $\mathrm{H}$ & -3.211964 & -3.109909 & 0.233502 \\
\hline $\mathrm{H}$ & -2.958221 & 3.130325 & -1.198089 \\
\hline $\mathrm{H}$ & 2.695055 & 2.984954 & 1.831947 \\
\hline $\mathrm{H}$ & 3.176028 & -3.123193 & -0.062918 \\
\hline $\mathrm{H}$ & -6.135489 & 1.012015 & -1.533581 \\
\hline $\mathrm{H}$ & -4.961868 & 2.094245 & -2.306309 \\
\hline $\mathrm{H}$ & -5.409095 & 2.338071 & -0.608012 \\
\hline $\mathrm{H}$ & -5.543177 & -1.861968 & 0.603253 \\
\hline $\mathrm{H}$ & -5.269196 & -2.444147 & -1.047855 \\
\hline $\mathrm{H}$ & -6.280429 & -1.014351 & -0.768359 \\
\hline $\mathrm{H}$ & -2.215702 & 5.667664 & 0.340672 \\
\hline $\mathrm{H}$ & -2.113600 & 5.150716 & -1.353925 \\
\hline $\mathrm{H}$ & -0.867069 & 6.219401 & -0.666616 \\
\hline $\mathrm{H}$ & 2.263284 & 5.014639 & 1.522472 \\
\hline $\mathrm{H}$ & -0.258530 & 6.786164 & 1.460771 \\
\hline $\mathrm{H}$ & 1.453055 & 7.205847 & 2.039819 \\
\hline $\mathrm{H}$ & 4.478854 & 2.139202 & 2.754008 \\
\hline
\end{tabular}




$\begin{array}{lrrr}\mathrm{H} & 5.616199 & 1.986020 & 1.399045 \\ \mathrm{H} & 5.612793 & 0.772591 & 2.689018 \\ \mathrm{H} & 5.045972 & -2.623012 & 0.685238 \\ \mathrm{H} & 6.875017 & -0.164769 & 1.024904 \\ \mathrm{H} & 7.337525 & -1.959129 & 0.920906 \\ \mathrm{H} & 2.272628 & -5.394079 & 0.767950 \\ \mathrm{H} & 2.274331 & -5.328894 & -1.004774 \\ \mathrm{H} & 1.075487 & -6.319844 & -0.152328 \\ \mathrm{H} & -1.067900 & -6.325157 & 0.197088 \\ \mathrm{H} & -2.390547 & -5.390123 & -0.524147 \\ \mathrm{H} & -2.125281 & -5.344127 & 1.228080 \\ \mathrm{C} & -0.342782 & -0.183258 & 1.823213 \\ \mathrm{~N} & -0.550211 & -0.286108 & 2.974112\end{array}$

His-heme-CN, $152.4^{\circ} \alpha$-meso-Fe- $\gamma$-meso angle

$\begin{array}{lrrr}\mathrm{C} & 0.500461 & -0.340256 & -4.282498 \\ \mathrm{~N} & 1.358902 & 0.700995 & -3.977771 \\ \mathrm{C} & -0.060510 & -0.713751 & -3.086430 \\ \mathrm{C} & 1.297619 & 0.923729 & -2.637973 \\ \mathrm{~N} & 0.443305 & 0.079374 & -2.075186 \\ \mathrm{H} & 0.372535 & -0.709049 & -5.293270 \\ \mathrm{H} & 1.938395 & 1.211373 & -4.635865 \\ \mathrm{H} & -0.783648 & -1.493945 & -2.877064 \\ \mathrm{H} & 1.869854 & 1.683561 & -2.116662 \\ \mathrm{C} & -2.422827 & -2.359274 & 0.107588 \\ \mathrm{C} & -2.238046 & 2.366140 & -0.907088 \\ \mathrm{C} & 2.056456 & 2.265589 & 1.308576 \\ \mathrm{C} & 2.399537 & -2.357602 & -0.053648 \\ \mathrm{C} & -2.812045 & -1.066237 & -0.223052 \\ \mathrm{C} & -4.166042 & -0.650459 & -0.531313 \\ \mathrm{C} & -4.090927 & 0.659386 & -0.950716 \\ \mathrm{C} & -2.698355 & 1.056478 & -0.833933 \\ \mathrm{C} & -5.199572 & 1.566088 & -1.378995 \\ \mathrm{C} & -5.371990 & -1.526109 & -0.411845 \\ \mathrm{C} & -1.015027 & 2.798438 & -0.389753 \\ \mathrm{C} & -0.700900 & 4.170461 & -0.056022 \\ \mathrm{C} & 0.460760 & 4.139374 & 0.702729 \\ \mathrm{C} & 0.875347 & 2.741501 & 0.753165 \\ \mathrm{C} & -1.510799 & 5.356225 & -0.464003 \\ \mathrm{C} & 1.204259 & 5.228948 & 1.308404 \\ \mathrm{C} & 0.760736 & 6.464007 & 1.617562 \\ \mathrm{C} & 2.592237 & 0.998338 & 1.062071 \\ \mathrm{C} & 3.947241 & 0.591117 & 1.349909 \\ \mathrm{C} & 4.095052 & -0.685550 & 0.819595 \\ \mathrm{C} & 2.782757 & -1.061437 & 0.300870 \\ \mathrm{C} & 4.950575 & 1.406254 & 2.097629 \\ \mathrm{C} & 5.258110 & -1.552870 & 0.803041 \\ \mathrm{C} & 6.552753 & -1.198785 & 0.934158 \\ \mathrm{C} & 1.085459 & -2.804147 & -0.150508 \\ \mathrm{C} & 0.679840 & -4.202844 & -0.081259 \\ \mathrm{C} & -0.683215 & -4.204051 & 0.098980 \\ \mathrm{C} & -1.103733 & -2.811207 & 0.068529\end{array}$




\begin{tabular}{|c|c|c|c|}
\hline $\mathrm{C}$ & 1.618735 & -5.364642 & -0.130022 \\
\hline C & -1.605692 & -5.366477 & 0.279729 \\
\hline $\mathrm{N}$ & -1.934840 & -0.016197 & -0.427703 \\
\hline $\mathrm{N}$ & -0.031843 & 1.954285 & 0.072334 \\
\hline $\mathrm{N}$ & 1.918522 & -0.005531 & 0.406803 \\
\hline $\mathrm{N}$ & -0.021149 & -1.991131 & -0.108690 \\
\hline $\mathrm{Fe}$ & -0.024929 & -0.007146 & -0.049774 \\
\hline $\mathrm{H}$ & -3.207928 & -3.100131 & 0.263253 \\
\hline $\mathrm{H}$ & -2.950606 & 3.129380 & -1.220104 \\
\hline $\mathrm{H}$ & 2.678988 & 2.973143 & 1.855162 \\
\hline $\mathrm{H}$ & 3.178413 & -3.119873 & -0.086320 \\
\hline $\mathrm{H}$ & -6.134689 & 1.011256 & -1.530638 \\
\hline $\mathrm{H}$ & -4.962981 & 2.080518 & -2.323858 \\
\hline $\mathrm{H}$ & -5.398803 & 2.346304 & -0.625821 \\
\hline $\mathrm{H}$ & -5.536766 & -1.843635 & 0.629985 \\
\hline $\mathrm{H}$ & -5.273921 & -2.440992 & -1.017460 \\
\hline $\mathrm{H}$ & -6.279858 & -1.006221 & -0.744801 \\
\hline $\mathrm{H}$ & -2.216629 & 5.664539 & 0.326053 \\
\hline $\mathrm{H}$ & -2.100252 & 5.153155 & -1.369451 \\
\hline $\mathrm{H}$ & -0.859533 & 6.219326 & -0.667992 \\
\hline $\mathrm{H}$ & 2.249544 & 5.006661 & 1.548320 \\
\hline $\mathrm{H}$ & -0.271659 & 6.778455 & 1.467985 \\
\hline $\mathrm{H}$ & 1.434012 & 7.195918 & 2.065736 \\
\hline $\mathrm{H}$ & 4.464247 & 2.122715 & 2.774281 \\
\hline $\mathrm{H}$ & 5.609286 & 1.981145 & 1.424423 \\
\hline $\mathrm{H}$ & 5.599496 & 0.757586 & 2.704816 \\
\hline $\mathrm{H}$ & 5.050210 & -2.618218 & 0.653857 \\
\hline $\mathrm{H}$ & 6.872880 & -0.162979 & 1.045011 \\
\hline $\mathrm{H}$ & 7.338766 & -1.954742 & 0.914860 \\
\hline $\mathrm{H}$ & 2.282797 & -5.387677 & 0.750321 \\
\hline $\mathrm{H}$ & 2.263838 & -5.332775 & -1.022652 \\
\hline $\mathrm{H}$ & 1.074359 & -6.317866 & -0.150484 \\
\hline $\mathrm{H}$ & -1.064003 & -6.319874 & 0.223411 \\
\hline $\mathrm{H}$ & -2.393809 & -5.389664 & -0.490910 \\
\hline $\mathrm{H}$ & -2.111192 & -5.331960 & 1.258179 \\
\hline $\mathrm{C}$ & -0.340124 & -0.182283 & 1.810287 \\
\hline $\mathrm{N}$ & -0.547471 & -0.285142 & 2.961167 \\
\hline \multicolumn{4}{|c|}{ His-heme-CN, $151.3^{\circ} \alpha$-meso-Fe- $\gamma$-meso angle } \\
\hline $\mathrm{C}$ & 0.500539 & -0.337371 & -4.295378 \\
\hline $\mathrm{N}$ & 1.364030 & 0.699387 & -3.989705 \\
\hline $\mathrm{C}$ & -0.060456 & -0.710825 & -3.099298 \\
\hline $\mathrm{C}$ & 1.303350 & 0.921617 & -2.649754 \\
\hline $\mathrm{N}$ & 0.445900 & 0.080044 & -2.087529 \\
\hline $\mathrm{H}$ & 0.370497 & -0.704347 & -5.306539 \\
\hline $\mathrm{H}$ & 1.945660 & 1.207853 & -4.647383 \\
\hline $\mathrm{H}$ & -0.786137 & -1.488753 & -2.890302 \\
\hline $\mathrm{H}$ & 1.878547 & 1.678791 & -2.127843 \\
\hline $\mathrm{C}$ & -2.418581 & -2.351831 & 0.123738 \\
\hline $\mathrm{C}$ & -2.231744 & 2.365143 & -0.926246 \\
\hline $\mathrm{C}$ & 2.046190 & 2.257102 & 1.319677 \\
\hline $\mathrm{C}$ & 2.401709 & -2.354581 & -0.07434 \\
\hline
\end{tabular}




\begin{tabular}{|c|c|c|c|}
\hline C & -2.808582 & -1.061469 & -0.217243 \\
\hline $\mathrm{C}$ & -4.163188 & -0.645613 & -0.523139 \\
\hline $\mathrm{C}$ & -4.087456 & 0.659913 & -0.956126 \\
\hline $\mathrm{C}$ & -2.693745 & 1.056025 & -0.848389 \\
\hline $\mathrm{C}$ & -5.196361 & 1.564528 & -1.388170 \\
\hline $\mathrm{C}$ & -5.370499 & -1.517242 & -0.389100 \\
\hline $\mathrm{C}$ & -1.010246 & 2.797561 & -0.404407 \\
\hline $\mathrm{C}$ & -0.699050 & 4.168338 & -0.062670 \\
\hline C & 0.455409 & 4.134369 & 0.707188 \\
\hline C & 0.870411 & 2.736541 & 0.755491 \\
\hline $\mathrm{C}$ & -1.505616 & 5.355573 & -0.473002 \\
\hline $\mathrm{C}$ & 1.192752 & 5.221645 & 1.324364 \\
\hline $\mathrm{C}$ & 0.746275 & 6.455647 & 1.633498 \\
\hline $\mathrm{C}$ & 2.587132 & 0.993354 & 1.064528 \\
\hline $\mathrm{C}$ & 3.941511 & 0.585621 & 1.354731 \\
\hline $\mathrm{C}$ & 4.094495 & -0.685395 & 0.811942 \\
\hline $\mathrm{C}$ & 2.784764 & -1.059264 & 0.284741 \\
\hline C & 4.940001 & 1.395418 & 2.114636 \\
\hline C & 5.259349 & -1.550017 & 0.790437 \\
\hline $\mathrm{C}$ & 6.552584 & -1.196305 & 0.936139 \\
\hline $\mathrm{C}$ & 1.087194 & -2.801929 & -0.166108 \\
\hline $\mathrm{C}$ & 0.681161 & -4.200064 & -0.087067 \\
\hline $\mathrm{C}$ & -0.680048 & -4.199411 & 0.107708 \\
\hline $\mathrm{C}$ & -1.100371 & -2.806529 & 0.074309 \\
\hline $\mathrm{C}$ & 1.618706 & -5.362819 & -0.139685 \\
\hline C & -1.600781 & -5.360540 & 0.305134 \\
\hline $\mathrm{N}$ & -1.930788 & -0.014987 & -0.436925 \\
\hline $\mathrm{N}$ & -0.029982 & 1.952590 & 0.061954 \\
\hline $\mathrm{N}$ & 1.919120 & -0.005256 & 0.395903 \\
\hline $\mathrm{N}$ & -0.018997 & -1.988741 & -0.118662 \\
\hline $\mathrm{Fe}$ & -0.022045 & -0.006531 & -0.063501 \\
\hline $\mathrm{H}$ & -3.203386 & -3.090136 & 0.292392 \\
\hline $\mathrm{H}$ & -2.943322 & 3.128412 & -1.241472 \\
\hline $\mathrm{H}$ & 2.662867 & 2.961125 & 1.877430 \\
\hline $\mathrm{H}$ & 3.180779 & -3.116600 & -0.109450 \\
\hline $\mathrm{H}$ & -6.134231 & 1.010764 & -1.526114 \\
\hline $\mathrm{H}$ & -4.964939 & 2.066013 & -2.341218 \\
\hline $\mathrm{H}$ & -5.387914 & 2.354903 & -0.643669 \\
\hline $\mathrm{H}$ & -5.529593 & -1.825688 & 0.656341 \\
\hline $\mathrm{H}$ & -5.278558 & -2.437403 & -0.987627 \\
\hline $\mathrm{H}$ & -6.279081 & -0.997821 & -0.720805 \\
\hline $\mathrm{H}$ & -2.217170 & 5.661741 & 0.312738 \\
\hline $\mathrm{H}$ & -2.088231 & 5.155459 & -1.383524 \\
\hline $\mathrm{H}$ & -0.852618 & 6.219095 & -0.669489 \\
\hline $\mathrm{H}$ & 2.235484 & 4.998245 & 1.574070 \\
\hline $\mathrm{H}$ & -0.284426 & 6.771006 & 1.474433 \\
\hline $\mathrm{H}$ & 1.415127 & 7.185764 & 2.091148 \\
\hline $\mathrm{H}$ & 4.449284 & 2.106457 & 2.793795 \\
\hline $\mathrm{H}$ & 5.602457 & 1.975580 & 1.449706 \\
\hline $\mathrm{H}$ & 5.585491 & 0.742499 & 2.720921 \\
\hline $\mathrm{H}$ & 5.054343 & -2.613180 & 0.622820 \\
\hline $\mathrm{H}$ & 6.870516 & -0.162133 & 1.066394 \\
\hline
\end{tabular}




$\begin{array}{lrrr}\mathrm{H} & 7.339687 & -1.950920 & 0.910034 \\ \mathrm{H} & 2.293306 & -5.380764 & 0.732762 \\ \mathrm{H} & 2.253056 & -5.337072 & -1.040159 \\ \mathrm{H} & 1.073503 & -6.315764 & -0.147495 \\ \mathrm{H} & -1.059486 & -6.314275 & 0.250575 \\ \mathrm{H} & -2.396253 & -5.389401 & -0.457689 \\ \mathrm{H} & -2.096916 & -5.319045 & 1.288077 \\ \mathrm{C} & -0.337541 & -0.181140 & 1.796935 \\ \mathrm{~N} & -0.544874 & -0.283932 & 2.947785\end{array}$

His-heme-CN, $150.3^{\circ} \alpha$-meso-Fe- $\gamma$-meso angle

$\begin{array}{lrrr}\mathrm{C} & 0.502406 & -0.335609 & -4.308405 \\ \mathrm{~N} & 1.368112 & 0.699118 & -4.002230 \\ \mathrm{C} & -0.060964 & -0.707052 & -3.112806 \\ \mathrm{C} & 1.308649 & 0.920155 & -2.661987 \\ \mathrm{~N} & 0.448575 & 0.080866 & -2.100301 \\ \mathrm{H} & 0.371681 & -0.701931 & -5.319711 \\ \mathrm{H} & 1.951060 & 1.206462 & -4.659599 \\ \mathrm{H} & -0.789603 & -1.482354 & -2.904348 \\ \mathrm{H} & 1.886353 & 1.675015 & -2.139499 \\ \mathrm{C} & -2.413995 & -2.344300 & 0.139434 \\ \mathrm{C} & -2.225579 & 2.364123 & -0.945083 \\ \mathrm{C} & 2.035942 & 2.248461 & 1.330135 \\ \mathrm{C} & 2.403926 & -2.351657 & -0.094914 \\ \mathrm{C} & -2.804910 & -1.056695 & -0.211770 \\ \mathrm{C} & -4.160178 & -0.640694 & -0.514954 \\ \mathrm{C} & -4.084021 & 0.660483 & -0.961204 \\ \mathrm{C} & -2.689192 & 1.055536 & -0.862703 \\ \mathrm{C} & -5.193309 & 1.563030 & -1.396630 \\ \mathrm{C} & -5.368734 & -1.508183 & -0.366264 \\ \mathrm{C} & -1.005524 & 2.796687 & -0.418989 \\ \mathrm{C} & -0.697356 & 4.166173 & -0.069191 \\ \mathrm{C} & 0.449982 & 4.129274 & 0.711424 \\ \mathrm{C} & 0.865521 & 2.731514 & 0.757372 \\ \mathrm{C} & -1.500799 & 5.354864 & -0.481455 \\ \mathrm{C} & 1.181127 & 5.214168 & 1.340002 \\ \mathrm{C} & 0.731908 & 6.447266 & 1.648811 \\ \mathrm{C} & 2.582034 & 0.988263 & 1.066566 \\ \mathrm{C} & 3.935698 & 0.579924 & 1.359397 \\ \mathrm{C} & 4.093866 & -0.685439 & 0.804503 \\ \mathrm{C} & 2.786811 & -1.057175 & 0.268636 \\ \mathrm{C} & 4.929235 & 1.384274 & 2.131465 \\ \mathrm{C} & 5.260405 & -1.547482 & 0.778515 \\ \mathrm{C} & 6.552150 & -1.194630 & 0.939195 \\ \mathrm{C} & 1.089007 & -2.799769 & -0.181798 \\ \mathrm{C} & 0.682640 & -4.197294 & -0.092856 \\ \mathrm{C} & -0.676597 & -4.194690 & 0.116220 \\ \mathrm{C} & -1.096773 & -2.801797 & 0.079683 \\ \mathrm{C} & 1.618813 & -5.361020 & -0.149064 \\ \mathrm{C} & -1.595412 & -5.354396 & 0.330383 \\ \mathrm{~N} & -1.926662 & -0.013863 & -0.446439 \\ \mathrm{~N} & -0.028037 & 1.950908 & 0.051295\end{array}$




\begin{tabular}{|c|c|c|c|}
\hline $\mathrm{N}$ & 1.919791 & -0.005025 & 0.38470 \\
\hline $\mathrm{N}$ & -0.016735 & -1.986364 & -0.12899 \\
\hline $\mathrm{Fe}$ & -0.019029 & -0.005955 & -0.07765 \\
\hline $\mathrm{H}$ & -3.198348 & -3.079931 & 0.32123 \\
\hline $\mathrm{H}$ & -2.936311 & 3.127423 & -1.2621 \\
\hline $\mathrm{H}$ & 2.646724 & 2.948845 & 1.8989 \\
\hline $\mathrm{H}$ & 3.183144 & -3.113473 & -0.1321 \\
\hline $\mathrm{H}$ & -6.134092 & 1.010717 & -1.5198 \\
\hline $\mathrm{H}$ & -4.967743 & 2.050907 & -2.35808 \\
\hline $\mathrm{H}$ & -5.376396 & 2.363903 & -0.6612 \\
\hline $\mathrm{H}$ & -5.521609 & -1.808097 & 0.6825 \\
\hline $\mathrm{H}$ & -5.283252 & -2.433228 & -0.9581 \\
\hline $\mathrm{H}$ & -6.278149 & -0.988960 & -0.6959 \\
\hline $\mathrm{H}$ & -2.217392 & 5.659123 & 0.3004 \\
\hline $\mathrm{H}$ & -2.077291 & 5.157573 & -1.3964 \\
\hline $\mathrm{H}$ & -0.846226 & 6.218708 & -0.6711 \\
\hline $\mathrm{H}$ & 2.221117 & 4.989463 & 1.5996 \\
\hline $\mathrm{H}$ & -0.296879 & 6.763800 & 1.4799 \\
\hline $\mathrm{H}$ & 1.396319 & 7.175465 & 2.1159 \\
\hline $\mathrm{H}$ & 4.434096 & 2.090306 & 2.8125 \\
\hline $\mathrm{H}$ & 5.595810 & 1.969204 & 1.4749 \\
\hline $\mathrm{H}$ & 5.570881 & 0.727169 & 2.7373 \\
\hline $\mathrm{H}$ & 5.058284 & -2.608125 & 0.5924 \\
\hline $\mathrm{H}$ & 6.867943 & -0.162496 & 1.088 \\
\hline $\mathrm{H}$ & 7.340232 & -1.947967 & 0.9065 \\
\hline $\mathrm{H}$ & 2.304136 & -5.373488 & 0.7151 \\
\hline $\mathrm{H}$ & 2.242028 & -5.341822 & -1.0574 \\
\hline $\mathrm{H}$ & 1.072958 & -6.313614 & -0.1436 \\
\hline $\mathrm{H}$ & -1.054376 & -6.308427 & 0.2782 \\
\hline $\mathrm{H}$ & -2.397828 & -5.389289 & $-0.424 \varepsilon$ \\
\hline $\mathrm{H}$ & -2.082573 & -5.305497 & 1.317 \\
\hline C & -0.335044 & -0.179862 & 1.783 \\
\hline $\mathrm{N}$ & -0.542477 & -0.282494 & 2.9339 \\
\hline \multicolumn{4}{|c|}{ His-heme-CN, $149.3^{\circ} \alpha$-meso-Fe- $\gamma$-meso angle } \\
\hline $\mathrm{C}$ & 0.502418 & -0.331599 & -4.3221 \\
\hline $\mathrm{N}$ & 1.373620 & 0.698081 & -4.0147 \\
\hline $\mathrm{C}$ & -0.061091 & -0.703203 & -3.1266 \\
\hline $\mathrm{C}$ & 1.314873 & 0.918169 & -2.6742 \\
\hline $\mathrm{N}$ & 0.451329 & 0.081856 & -2.1133 \\
\hline $\mathrm{H}$ & 0.369390 & -0.695662 & -5.3340 \\
\hline $\mathrm{H}$ & 1.958909 & 1.203467 & -4.6715 \\
\hline $\mathrm{H}$ & -0.792612 & -1.475961 & -2.9188 \\
\hline $\mathrm{H}$ & 1.895875 & 1.669909 & -2.15093 \\
\hline $\mathrm{C}$ & -2.409059 & -2.336662 & 0.15474 \\
\hline $\mathrm{C}$ & -2.219640 & 2.363069 & -0.9636 \\
\hline $\mathrm{C}$ & 2.025568 & 2.239683 & 1.3400 \\
\hline $\mathrm{C}$ & 2.406191 & -2.348795 & -0.1152 \\
\hline $\mathrm{C}$ & -2.801046 & -1.051908 & -0.2065 \\
\hline $\mathrm{C}$ & -4.157038 & -0.635757 & -0.5067 \\
\hline $\mathrm{C}$ & -4.080690 & 0.661042 & -0.9659 \\
\hline $\mathrm{C}$ & -2.684772 & 1.054988 & $-0.876 s$ \\
\hline
\end{tabular}




\begin{tabular}{|c|c|c|c|}
\hline $\mathrm{C}$ & -5.190510 & 1.561505 & -1.404400 \\
\hline $\mathrm{C}$ & -5.366675 & -1.499051 & -0.343361 \\
\hline $\mathrm{C}$ & -1.000948 & 2.795825 & -0.433542 \\
\hline $\mathrm{C}$ & -0.695903 & 4.163986 & -0.075666 \\
\hline $\mathrm{C}$ & 0.444395 & 4.124118 & 0.715374 \\
\hline $\mathrm{C}$ & 0.860547 & 2.726440 & 0.758834 \\
\hline $\mathrm{C}$ & -1.496395 & 5.354114 & -0.489549 \\
\hline C & 1.169321 & 5.206557 & 1.355218 \\
\hline C & 0.717558 & 6.438887 & 1.663439 \\
\hline C & 2.576848 & 0.983101 & 1.068250 \\
\hline $\mathrm{C}$ & 3.929726 & 0.574116 & 1.363947 \\
\hline $\mathrm{C}$ & 4.093151 & -0.685577 & 0.797290 \\
\hline C & 2.788875 & -1.055116 & 0.252627 \\
\hline $\mathrm{C}$ & 4.918185 & 1.372969 & 2.148119 \\
\hline $\mathrm{C}$ & 5.261312 & -1.545107 & 0.767243 \\
\hline C & 6.551474 & -1.193479 & 0.943034 \\
\hline C & 1.090896 & -2.797648 & -0.197450 \\
\hline C & 0.684286 & -4.194512 & -0.098514 \\
\hline C & -0.672861 & -4.189878 & 0.124601 \\
\hline $\mathrm{C}$ & -1.092930 & -2.797002 & 0.084739 \\
\hline $\mathrm{C}$ & 1.619072 & -5.359219 & -0.158065 \\
\hline $\mathrm{C}$ & -1.589621 & -5.348041 & 0.355404 \\
\hline $\mathrm{N}$ & -1.922524 & -0.012827 & -0.456238 \\
\hline $\mathrm{N}$ & -0.026111 & 1.949256 & 0.040385 \\
\hline $\mathrm{N}$ & 1.920480 & -0.004809 & 0.373262 \\
\hline $\mathrm{N}$ & -0.014367 & -1.984001 & -0.139570 \\
\hline $\mathrm{Fe}$ & -0.015945 & -0.005422 & -0.092154 \\
\hline $\mathrm{H}$ & -3.192807 & -3.069515 & 0.349742 \\
\hline $\mathrm{H}$ & -2.929659 & 3.126390 & -1.282336 \\
\hline $\mathrm{H}$ & 2.630428 & 2.936357 & 1.919648 \\
\hline $\mathrm{H}$ & 3.185532 & -3.110440 & -0.154364 \\
\hline $\mathrm{H}$ & -6.134246 & 1.010964 & -1.512021 \\
\hline $\mathrm{H}$ & -4.971394 & 2.035333 & -2.374309 \\
\hline $\mathrm{H}$ & -5.364583 & 2.373022 & -0.678640 \\
\hline $\mathrm{H}$ & -5.512804 & -1.791011 & 0.708702 \\
\hline $\mathrm{H}$ & -5.287928 & -2.428580 & -0.929144 \\
\hline $\mathrm{H}$ & -6.277048 & -0.979781 & -0.670323 \\
\hline $\mathrm{H}$ & -2.217467 & 5.656691 & 0.288870 \\
\hline $\mathrm{H}$ & -2.067351 & 5.159515 & -1.408603 \\
\hline $\mathrm{H}$ & -0.840358 & 6.218188 & -0.673085 \\
\hline $\mathrm{H}$ & 2.206388 & 4.980376 & 1.625033 \\
\hline $\mathrm{H}$ & -0.309119 & 6.756813 & 1.484703 \\
\hline $\mathrm{H}$ & 1.377501 & 7.165062 & 2.139957 \\
\hline $\mathrm{H}$ & 4.418590 & 2.074294 & 2.830820 \\
\hline $\mathrm{H}$ & 5.589104 & 1.962306 & 1.500001 \\
\hline $\mathrm{H}$ & 5.555720 & 0.711756 & 2.753843 \\
\hline $\mathrm{H}$ & 5.062125 & -2.602946 & 0.563037 \\
\hline $\mathrm{H}$ & 6.865112 & -0.163728 & 1.112030 \\
\hline $\mathrm{H}$ & 7.340486 & -1.945548 & 0.904158 \\
\hline $\mathrm{H}$ & 2.315167 & -5.365969 & 0.697547 \\
\hline $\mathrm{H}$ & 2.230896 & -5.346885 & -1.074229 \\
\hline $\mathrm{H}$ & 1.072743 & -6.311373 & -0.138931 \\
\hline
\end{tabular}




$\begin{array}{lllr}\mathrm{H} & -1.048711 & -6.302317 & 0.306385 \\ \mathrm{H} & -2.398486 & -5.389250 & -0.392553 \\ \mathrm{H} & -2.068318 & -5.291382 & 1.346178 \\ \mathrm{C} & -0.332722 & -0.178427 & 1.769053 \\ \mathrm{~N} & -0.540384 & -0.280786 & 2.919810\end{array}$

His-heme-CN, $148.3^{\circ} \alpha$-meso-Fe- $\gamma$-meso angle

\begin{tabular}{|c|c|c|c|}
\hline $\mathrm{C}$ & 0.504292 & -0.329241 & -4.335853 \\
\hline $\mathrm{N}$ & 1.378217 & 0.697865 & -4.027656 \\
\hline $\mathrm{C}$ & -0.061794 & -0.698784 & -3.140924 \\
\hline C & 1.320744 & 0.916504 & -2.686839 \\
\hline $\mathrm{N}$ & 0.454151 & 0.082821 & -2.126704 \\
\hline $\mathrm{H}$ & 0.370414 & -0.692360 & -5.347897 \\
\hline $\mathrm{H}$ & 1.965110 & 1.201945 & -4.684005 \\
\hline $\mathrm{H}$ & -0.796686 & -1.468536 & -2.933875 \\
\hline $\mathrm{H}$ & 1.904636 & 1.665489 & -2.162796 \\
\hline $\mathrm{C}$ & -2.403798 & -2.328832 & 0.169769 \\
\hline C & -2.213822 & 2.362045 & -0.981917 \\
\hline C & 2.015135 & 2.230748 & 1.349440 \\
\hline C & 2.408499 & -2.346016 & -0.135315 \\
\hline C & -2.796983 & -1.047017 & -0.201602 \\
\hline $\mathrm{C}$ & -4.153738 & -0.630695 & -0.498505 \\
\hline C & -4.077387 & 0.661687 & -0.970429 \\
\hline C & -2.680414 & 1.054462 & -0.890953 \\
\hline C & -5.187846 & 1.560056 & -1.411594 \\
\hline C & -5.364303 & -1.489744 & -0.320456 \\
\hline C & -0.996419 & 2.795003 & -0.448006 \\
\hline C & -0.694583 & 4.161789 & -0.081986 \\
\hline C & 0.438700 & 4.118871 & 0.719227 \\
\hline C & 0.855540 & 2.721291 & 0.760012 \\
\hline C & -1.492228 & 5.353376 & -0.497197 \\
\hline $\mathrm{C}$ & 1.157359 & 5.198757 & 1.370277 \\
\hline C & 0.703229 & 6.430427 & 1.677730 \\
\hline C & 2.571638 & 0.977857 & 1.069624 \\
\hline C & 3.923676 & 0.568186 & 1.368325 \\
\hline $\mathrm{C}$ & 4.092396 & -0.685820 & 0.790229 \\
\hline C & 2.790983 & -1.053102 & 0.236710 \\
\hline C & 4.906970 & 1.361488 & 2.164491 \\
\hline C & 5.262130 & -1.542889 & 0.756470 \\
\hline C & 6.550624 & -1.192786 & 0.947273 \\
\hline C & 1.092849 & -2.795567 & -0.212985 \\
\hline C & 0.686040 & -4.191710 & -0.104030 \\
\hline C & -0.668914 & -4.184932 & 0.132836 \\
\hline C & -1.088879 & -2.792092 & 0.089582 \\
\hline $\mathrm{C}$ & 1.619382 & -5.357439 & -0.166735 \\
\hline C & -1.583574 & -5.341408 & 0.379935 \\
\hline $\mathrm{N}$ & -1.918337 & -0.011798 & -0.46617 \\
\hline $\mathrm{N}$ & -0.024126 & 1.947639 & 0.02928 \\
\hline $\mathrm{N}$ & 1.921222 & -0.004621 & 0.36165 \\
\hline $\mathrm{N}$ & -0.011897 & -1.981623 & -0.150261 \\
\hline $\mathrm{Fe}$ & -0.012759 & -0.004928 & -0.10688 \\
\hline $\mathrm{H}$ & -3.186804 & -3.058792 & 0.37799 \\
\hline
\end{tabular}




\begin{tabular}{|c|c|c|c|}
\hline & & & \\
\hline $\mathrm{H}$ & -2.923236 & 3.125388 & -1.301943 \\
\hline $\mathrm{H}$ & 2.614037 & 2.923627 & 1.939757 \\
\hline $\mathrm{H}$ & 3.187926 & -3.107532 & -0.176059 \\
\hline $\mathrm{H}$ & -6.134453 & 1.011544 & -1.503337 \\
\hline $\mathrm{H}$ & -4.975523 & 2.019698 & -2.389775 \\
\hline $\mathrm{H}$ & -5.352699 & 2.382111 & -0.695636 \\
\hline $\mathrm{H}$ & -5.503225 & -1.774369 & 0.734598 \\
\hline $\mathrm{H}$ & -5.292516 & -2.423346 & -0.900625 \\
\hline $\mathrm{H}$ & -6.275725 & -0.970167 & -0.643983 \\
\hline $\mathrm{H}$ & -2.217342 & 5.654440 & 0.278052 \\
\hline $\mathrm{H}$ & -2.058104 & 5.161420 & -1.419929 \\
\hline $\mathrm{H}$ & -0.834791 & 6.217597 & -0.674999 \\
\hline $\mathrm{H}$ & 2.191331 & 4.970939 & 1.650318 \\
\hline $\mathrm{H}$ & -0.321136 & 6.749954 & 1.488905 \\
\hline $\mathrm{H}$ & 1.358666 & 7.154462 & 2.163655 \\
\hline $\mathrm{H}$ & 4.402907 & 2.058299 & 2.848493 \\
\hline $\mathrm{H}$ & 5.582332 & 1.954991 & 1.524860 \\
\hline $\mathrm{H}$ & 5.540245 & 0.696228 & 2.770264 \\
\hline $\mathrm{H}$ & 5.065925 & -2.597684 & 0.534457 \\
\hline $\mathrm{H}$ & 6.862080 & -0.165712 & 1.135151 \\
\hline $\mathrm{H}$ & 7.340537 & -1.943581 & 0.902422 \\
\hline $\mathrm{H}$ & 2.326096 & -5.358473 & 0.680174 \\
\hline $\mathrm{H}$ & 2.219764 & -5.352104 & -1.090483 \\
\hline $\mathrm{H}$ & 1.072712 & -6.309034 & -0.133873 \\
\hline $\mathrm{H}$ & -1.042706 & -6.295898 & 0.334553 \\
\hline $\mathrm{H}$ & -2.398337 & -5.389021 & -0.361171 \\
\hline $\mathrm{H}$ & -2.054411 & -5.276774 & 1.373971 \\
\hline C & -0.330513 & -0.176797 & 1.754727 \\
\hline $\mathrm{N}$ & -0.538518 & -0.278748 & 2.905423 \\
\hline is $-\mathrm{r}$ & $\mathrm{N}, 147.3^{\circ} \alpha-\mathrm{me}$ & $\mathrm{e}-\gamma$-meso angle & \\
\hline $\mathrm{C}$ & 0.504362 & -0.325364 & -4.350146 \\
\hline $\mathrm{N}$ & 1.384295 & 0.696162 & -4.040651 \\
\hline C & -0.062136 & -0.694717 & -3.155341 \\
\hline $\mathrm{C}$ & 1.327573 & 0.913918 & -2.699607 \\
\hline $\mathrm{N}$ & 0.457043 & 0.083735 & -2.140305 \\
\hline $\mathrm{H}$ & 0.368042 & -0.686148 & -5.362697 \\
\hline $\mathrm{H}$ & 1.973843 & 1.197908 & -4.696395 \\
\hline $\mathrm{H}$ & -0.800361 & -1.461458 & -2.948999 \\
\hline $\mathrm{H}$ & 1.915168 & 1.659419 & -2.174747 \\
\hline C & -2.398243 & -2.320811 & 0.184472 \\
\hline C & -2.208178 & 2.361072 & -0.999915 \\
\hline $\mathrm{C}$ & 2.004636 & 2.221758 & 1.358332 \\
\hline C & 2.410850 & -2.343302 & -0.154958 \\
\hline C & -2.792747 & -1.042026 & -0.196886 \\
\hline $\mathrm{C}$ & -4.150291 & -0.625524 & -0.490360 \\
\hline $\mathrm{C}$ & -4.074122 & 0.662413 & -0.974712 \\
\hline C & -2.676140 & 1.053963 & -0.904901 \\
\hline C & -5.185327 & 1.558688 & -1.418313 \\
\hline C & -5.361622 & -1.480304 & -0.297756 \\
\hline C & -0.991991 & 2.794264 & -0.462403 \\
\hline 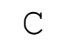 & -0.693530 & 4.159622 & -0.088113 \\
\hline
\end{tabular}




\begin{tabular}{|c|c|c|c|}
\hline $\mathrm{C}$ & 0.432815 & 4.113610 & 0.722970 \\
\hline $\mathrm{C}$ & 0.850487 & 2.716163 & 0.760841 \\
\hline $\mathrm{C}$ & -1.488567 & 5.352629 & -0.504274 \\
\hline $\mathrm{C}$ & 1.145120 & 5.190861 & 1.385204 \\
\hline $\mathrm{C}$ & 0.688668 & 6.421863 & 1.691975 \\
\hline $\mathrm{C}$ & 2.566425 & 0.972643 & 1.070615 \\
\hline $\mathrm{C}$ & 3.917579 & 0.562271 & 1.372463 \\
\hline $\mathrm{C}$ & 4.091613 & -0.686089 & 0.783354 \\
\hline C & 2.793135 & -1.051093 & 0.220968 \\
\hline C & 4.895673 & 1.350035 & 2.180403 \\
\hline $\mathrm{C}$ & 5.262870 & -1.540765 & 0.746296 \\
\hline $\mathrm{C}$ & 6.549632 & -1.192360 & 0.951675 \\
\hline $\mathrm{C}$ & 1.094859 & -2.793509 & -0.228264 \\
\hline $\mathrm{C}$ & 0.687893 & -4.188869 & -0.109245 \\
\hline $\mathrm{C}$ & -0.664780 & -4.179833 & 0.141027 \\
\hline $\mathrm{C}$ & -1.084635 & -2.787045 & 0.094239 \\
\hline $\mathrm{C}$ & 1.619751 & -5.355657 & -0.174812 \\
\hline $\mathrm{C}$ & -1.577326 & -5.334479 & 0.404068 \\
\hline $\mathrm{N}$ & -1.914125 & -0.010768 & -0.476336 \\
\hline $\mathrm{N}$ & -0.022089 & 1.946128 & 0.017926 \\
\hline $\mathrm{N}$ & 1.922034 & -0.004389 & 0.349848 \\
\hline $\mathrm{N}$ & -0.009328 & -1.979212 & -0.161007 \\
\hline $\mathrm{Fe}$ & -0.009463 & -0.004421 & -0.121897 \\
\hline $\mathrm{H}$ & -3.180373 & -3.047775 & 0.405875 \\
\hline $\mathrm{H}$ & -2.917121 & 3.124429 & -1.321006 \\
\hline $\mathrm{H}$ & 2.597531 & 2.910766 & 1.959196 \\
\hline $\mathrm{H}$ & 3.190332 & -3.104734 & -0.197039 \\
\hline $\mathrm{H}$ & -6.134571 & 1.012316 & -1.494500 \\
\hline $\mathrm{H}$ & -4.979863 & 2.004529 & -2.404285 \\
\hline $\mathrm{H}$ & -5.341200 & 2.390786 & -0.712042 \\
\hline $\mathrm{H}$ & -5.492928 & -1.758210 & 0.760068 \\
\hline $\mathrm{H}$ & -5.296983 & -2.417579 & -0.872819 \\
\hline $\mathrm{H}$ & -6.274164 & -0.960164 & -0.617201 \\
\hline $\mathrm{H}$ & -2.217426 & 5.652100 & 0.268077 \\
\hline $\mathrm{H}$ & -2.049693 & 5.163315 & -1.430433 \\
\hline $\mathrm{H}$ & -0.829899 & 6.217044 & -0.676574 \\
\hline $\mathrm{H}$ & 2.175888 & 4.961336 & 1.675409 \\
\hline $\mathrm{H}$ & -0.333268 & 6.743066 & 1.493139 \\
\hline $\mathrm{H}$ & 1.339520 & 7.143664 & 2.187318 \\
\hline $\mathrm{H}$ & 4.387206 & 2.042507 & 2.865519 \\
\hline $\mathrm{H}$ & 5.575451 & 1.947503 & 1.549204 \\
\hline $\mathrm{H}$ & 5.524663 & 0.680814 & 2.786287 \\
\hline $\mathrm{H}$ & 5.069674 & -2.592375 & 0.507239 \\
\hline $\mathrm{H}$ & 6.858869 & -0.168146 & 1.157602 \\
\hline $\mathrm{H}$ & 7.340440 & -1.941864 & 0.901249 \\
\hline $\mathrm{H}$ & 2.336610 & -5.351166 & 0.663531 \\
\hline $\mathrm{H}$ & 2.208996 & -5.357257 & -1.105695 \\
\hline $\mathrm{H}$ & 1.072848 & -6.306575 & -0.128586 \\
\hline $\mathrm{H}$ & -1.036426 & -6.289146 & 0.362838 \\
\hline $\mathrm{H}$ & -2.397439 & -5.388546 & -0.330624 \\
\hline $\mathrm{H}$ & -2.040914 & -5.261666 & 1.400930 \\
\hline $\mathrm{C}$ & -0.328360 & -0.174802 & 1.740147 \\
\hline
\end{tabular}




\begin{tabular}{|c|c|c|c|}
\hline \multirow{2}{*}{\multicolumn{4}{|c|}{$\begin{array}{ccc}\mathrm{N} & -0.536792 & -0.276121 \\
\text { His-heme-CN, 146.3 } & \alpha \text {-meso-Fe- } \gamma \text {-meso angle }\end{array}$}} \\
\hline & & & \\
\hline $\mathrm{C}$ & 0.505844 & -0.323072 & -4.364343 \\
\hline $\mathrm{N}$ & 1.389345 & 0.695112 & -4.054169 \\
\hline $\mathrm{C}$ & -0.063410 & -0.689902 & -3.170057 \\
\hline $\mathrm{C}$ & 1.334175 & 0.911432 & -2.712771 \\
\hline $\mathrm{N}$ & 0.459980 & 0.084612 & -2.154125 \\
\hline $\mathrm{H}$ & 0.368287 & -0.682766 & -5.377110 \\
\hline $\mathrm{H}$ & 1.980854 & 1.195051 & -4.709515 \\
\hline $\mathrm{H}$ & -0.805630 & -1.452965 & -2.964445 \\
\hline $\mathrm{H}$ & 1.925336 & 1.653631 & -2.187248 \\
\hline $\mathrm{C}$ & -2.392293 & -2.312581 & 0.198987 \\
\hline $\mathrm{C}$ & -2.202615 & 2.360151 & -1.017643 \\
\hline $\mathrm{C}$ & 1.994166 & 2.212714 & 1.366735 \\
\hline $\mathrm{C}$ & 2.413279 & -2.340674 & -0.174234 \\
\hline $\mathrm{C}$ & -2.788248 & -1.036913 & -0.192287 \\
\hline $\mathrm{C}$ & -4.146611 & -0.620231 & -0.482139 \\
\hline $\mathrm{C}$ & -4.070824 & 0.663227 & -0.978686 \\
\hline $\mathrm{C}$ & -2.671879 & 1.053505 & -0.918667 \\
\hline $\mathrm{C}$ & -5.182892 & 1.557397 & -1.424430 \\
\hline $\mathrm{C}$ & -5.358520 & -1.470779 & -0.275123 \\
\hline $\mathrm{C}$ & -0.987557 & 2.793599 & -0.476764 \\
\hline $\mathrm{C}$ & -0.692562 & 4.157488 & -0.094173 \\
\hline $\mathrm{C}$ & 0.426869 & 4.108321 & 0.726547 \\
\hline $\mathrm{C}$ & 0.845480 & 2.711035 & 0.761331 \\
\hline $\mathrm{C}$ & -1.485128 & 5.351906 & -0.511029 \\
\hline $\mathrm{C}$ & 1.132727 & 5.182836 & 1.399956 \\
\hline $\mathrm{C}$ & 0.674032 & 6.413198 & 1.706053 \\
\hline $\mathrm{C}$ & 2.561256 & 0.967432 & 1.071314 \\
\hline $\mathrm{C}$ & 3.911458 & 0.556286 & 1.376480 \\
\hline $\mathrm{C}$ & 4.090757 & -0.686491 & 0.776788 \\
\hline $\mathrm{C}$ & 2.795324 & -1.049131 & 0.205462 \\
\hline $\mathrm{C}$ & 4.884336 & 1.338478 & 2.196010 \\
\hline $\mathrm{C}$ & 5.263389 & -1.538966 & 0.736941 \\
\hline $\mathrm{C}$ & 6.548374 & -1.192430 & 0.956323 \\
\hline $\mathrm{C}$ & 1.096978 & -2.791495 & -0.243368 \\
\hline $\mathrm{C}$ & 0.689890 & -4.186013 & -0.114305 \\
\hline $\mathrm{C}$ & -0.660415 & -4.174612 & 0.149076 \\
\hline $\mathrm{C}$ & -1.080138 & -2.781884 & 0.098728 \\
\hline $\mathrm{C}$ & 1.620216 & -5.353897 & -0.182514 \\
\hline $\mathrm{C}$ & -1.570867 & -5.327297 & 0.427596 \\
\hline $\mathrm{N}$ & -1.909820 & -0.009721 & -0.486592 \\
\hline $\mathrm{N}$ & -0.019928 & 1.944705 & 0.006360 \\
\hline $\mathrm{N}$ & 1.922931 & -0.004139 & 0.337943 \\
\hline $\mathrm{N}$ & -0.006599 & -1.976772 & -0.171785 \\
\hline $\mathrm{Fe}$ & -0.006010 & -0.003907 & -0.137115 \\
\hline $\mathrm{H}$ & -3.173425 & -3.036433 & 0.433541 \\
\hline $\mathrm{H}$ & -2.911210 & 3.123510 & -1.339550 \\
\hline $\mathrm{H}$ & 2.580997 & 2.897777 & 1.978010 \\
\hline $\mathrm{H}$ & 3.192800 & -3.102046 & -0.217389 \\
\hline $\mathrm{H}$ & -6.134441 & 1.013127 & -1.485842 \\
\hline
\end{tabular}




\begin{tabular}{|c|c|c|c|}
\hline $\mathrm{H}$ & -4.984140 & 1.990181 & -2.417543 \\
\hline $\mathrm{H}$ & -5.330350 & 2.398798 & -0.727467 \\
\hline $\mathrm{H}$ & -5.481866 & -1.742582 & 0.785248 \\
\hline $\mathrm{H}$ & -5.301111 & -2.411343 & -0.845565 \\
\hline $\mathrm{H}$ & -6.272251 & -0.949881 & -0.589919 \\
\hline $\mathrm{H}$ & -2.217491 & 5.649791 & 0.258619 \\
\hline $\mathrm{H}$ & -2.041761 & 5.165231 & -1.440414 \\
\hline $\mathrm{H}$ & -0.825320 & 6.216510 & -0.678032 \\
\hline $\mathrm{H}$ & 2.160158 & 4.951505 & 1.700314 \\
\hline $\mathrm{H}$ & -0.345331 & 6.736186 & 1.497203 \\
\hline $\mathrm{H}$ & 1.320224 & 7.132667 & 2.210824 \\
\hline $\mathrm{H}$ & 4.371518 & 2.026702 & 2.882138 \\
\hline $\mathrm{H}$ & 5.568469 & 1.939788 & 1.573221 \\
\hline $\mathrm{H}$ & 5.509055 & 0.665335 & 2.801984 \\
\hline $\mathrm{H}$ & 5.073131 & -2.587326 & 0.481839 \\
\hline $\mathrm{H}$ & 6.855421 & -0.171174 & 1.179210 \\
\hline $\mathrm{H}$ & 7.340039 & -1.940680 & 0.900857 \\
\hline $\mathrm{H}$ & 2.346535 & -5.344306 & 0.647615 \\
\hline $\mathrm{H}$ & 2.198888 & -5.362124 & -1.119947 \\
\hline $\mathrm{H}$ & 1.073143 & -6.304035 & -0.123689 \\
\hline $\mathrm{H}$ & -1.029905 & -6.282117 & 0.390815 \\
\hline $\mathrm{H}$ & -2.395861 & -5.387704 & -0.301072 \\
\hline $\mathrm{H}$ & -2.027741 & -5.246259 & 1.426909 \\
\hline $\mathrm{C}$ & -0.326275 & -0.172516 & 1.725378 \\
\hline $\mathrm{N}$ & -0.535262 & -0.273033 & 2.875955 \\
\hline \multicolumn{4}{|c|}{ His-heme- $\mathrm{CN}, 145.3^{\circ} \alpha$-meso-Fe- $\gamma$-meso angle } \\
\hline $\mathrm{C}$ & 0.505625 & -0.319619 & -4.378875 \\
\hline $\mathrm{N}$ & 1.395673 & 0.692451 & -4.067603 \\
\hline $\mathrm{C}$ & -0.064154 & -0.685803 & -3.184629 \\
\hline $\mathrm{C}$ & 1.341496 & 0.908042 & -2.725988 \\
\hline $\mathrm{N}$ & 0.462977 & 0.085315 & -2.167999 \\
\hline $\mathrm{H}$ & 0.365344 & -0.676980 & -5.392092 \\
\hline $\mathrm{H}$ & 1.990051 & 1.189614 & -4.722450 \\
\hline $\mathrm{H}$ & -0.810139 & -1.445338 & -2.979611 \\
\hline $\mathrm{H}$ & 1.936839 & 1.646406 & -2.199801 \\
\hline $\mathrm{C}$ & -2.386199 & -2.304165 & 0.213321 \\
\hline $\mathrm{C}$ & -2.197132 & 2.359261 & -1.035078 \\
\hline $\mathrm{C}$ & 1.983602 & 2.203599 & 1.374902 \\
\hline $\mathrm{C}$ & 2.415708 & -2.338126 & -0.192905 \\
\hline $\mathrm{C}$ & -2.783652 & -1.031678 & -0.187809 \\
\hline C & -4.142828 & -0.614786 & -0.473988 \\
\hline $\mathrm{C}$ & -4.067527 & 0.664156 & -0.982496 \\
\hline C & -2.667657 & 1.053068 & -0.932273 \\
\hline C & -5.180502 & 1.556260 & -1.430169 \\
\hline $\mathrm{C}$ & -5.355190 & -1.461111 & -0.252838 \\
\hline $\mathrm{C}$ & -0.983147 & 2.792992 & -0.490989 \\
\hline C & -0.691763 & 4.155364 & -0.100033 \\
\hline C & 0.420777 & 4.103004 & 0.730115 \\
\hline C & 0.840425 & 2.705913 & 0.761695 \\
\hline $\mathrm{C}$ & -1.482001 & 5.351166 & -0.517373 \\
\hline . & 1.120101 & 5.174715 & 1.414641 \\
\hline
\end{tabular}




\begin{tabular}{|c|c|c|c|}
\hline C & 0.659215 & 6.404452 & 1.720066 \\
\hline C & 2.556090 & 0.962266 & 1.071830 \\
\hline $\mathrm{C}$ & 3.905339 & 0.550373 & 1.380298 \\
\hline C & 4.089943 & -0.686847 & 0.770385 \\
\hline C & 2.797564 & -1.047175 & 0.190253 \\
\hline C & 4.872986 & 1.327025 & 2.211161 \\
\hline $\mathrm{C}$ & 5.264039 & -1.537035 & 0.728021 \\
\hline C & 6.547226 & -1.192191 & 0.960468 \\
\hline C & 1.099089 & -2.789537 & -0.258004 \\
\hline C & 0.691871 & -4.183153 & -0.118985 \\
\hline C & -0.656020 & -4.169276 & 0.157076 \\
\hline $\mathrm{C}$ & -1.075586 & -2.776616 & 0.103162 \\
\hline C & 1.620599 & -5.352199 & -0.189576 \\
\hline $\mathrm{C}$ & -1.564462 & -5.319910 & 0.450392 \\
\hline $\mathrm{N}$ & -1.905508 & -0.008687 & -0.496886 \\
\hline $\mathrm{N}$ & -0.017687 & 1.943354 & -0.005270 \\
\hline $\mathrm{N}$ & 1.923888 & -0.003853 & 0.326058 \\
\hline $\mathrm{N}$ & -0.003853 & -1.974343 & -0.182397 \\
\hline $\mathrm{Fe}$ & -0.002465 & -0.003432 & -0.152399 \\
\hline $\mathrm{H}$ & -3.166244 & -3.024814 & 0.460838 \\
\hline $\mathrm{H}$ & -2.905477 & 3.122617 & -1.357612 \\
\hline $\mathrm{H}$ & 2.564313 & 2.884643 & 1.996461 \\
\hline $\mathrm{H}$ & 3.195249 & -3.099476 & -0.236877 \\
\hline $\mathrm{H}$ & -6.134004 & 1.013983 & -1.477956 \\
\hline $\mathrm{H}$ & -4.988081 & 1.976962 & -2.429675 \\
\hline $\mathrm{H}$ & -5.320324 & 2.406091 & -0.741949 \\
\hline $\mathrm{H}$ & -5.470417 & -1.727336 & 0.809863 \\
\hline $\mathrm{H}$ & -5.305018 & -2.404635 & -0.819060 \\
\hline $\mathrm{H}$ & -6.270103 & -0.939264 & -0.562614 \\
\hline $\mathrm{H}$ & -2.217692 & 5.647438 & 0.249721 \\
\hline $\mathrm{H}$ & -2.034327 & 5.167122 & -1.449831 \\
\hline $\mathrm{H}$ & -0.821152 & 6.215977 & -0.679224 \\
\hline $\mathrm{H}$ & 2.144077 & 4.941502 & 1.725114 \\
\hline $\mathrm{H}$ & -0.357452 & 6.729296 & 1.501242 \\
\hline $\mathrm{H}$ & 1.300660 & 7.121516 & 2.234246 \\
\hline $\mathrm{H}$ & 4.355868 & 2.010851 & 2.898438 \\
\hline $\mathrm{H}$ & 5.561148 & 1.932318 & 1.596723 \\
\hline $\mathrm{H}$ & 5.493727 & 0.650021 & 2.816937 \\
\hline $\mathrm{H}$ & 5.076811 & -2.582189 & 0.457979 \\
\hline $\mathrm{H}$ & 6.851949 & -0.173848 & 1.199051 \\
\hline $\mathrm{H}$ & 7.339856 & -1.939064 & 0.900329 \\
\hline $\mathrm{H}$ & 2.355471 & -5.338117 & 0.632935 \\
\hline $\mathrm{H}$ & 2.189544 & -5.366581 & -1.132842 \\
\hline $\mathrm{H}$ & 1.073335 & -6.301459 & -0.119257 \\
\hline $\mathrm{H}$ & -1.023436 & -6.274862 & 0.418196 \\
\hline $\mathrm{H}$ & -2.393858 & -5.386386 & -0.272689 \\
\hline $\mathrm{H}$ & -2.015188 & -5.230779 & 1.451793 \\
\hline C & -0.324302 & -0.169953 & 1.710563 \\
\hline $\mathrm{N}$ & -0.533958 & -0.269477 & 2.861073 \\
\hline \multicolumn{4}{|c|}{ is-heme-CN, $144.2^{\circ} \alpha$-meso- $\mathrm{Fe}-\gamma$-meso angle } \\
\hline $\mathrm{C}$ & 0.507137 & -0.317559 & -4.393222 \\
\hline
\end{tabular}




\begin{tabular}{|c|c|c|c|}
\hline $\mathrm{N}$ & 1.401146 & 0.690757 & -4.081311 \\
\hline C & -0.065521 & -0.680919 & -3.199477 \\
\hline C & 1.348588 & 0.904970 & -2.739348 \\
\hline $\mathrm{N}$ & 0.466087 & 0.086033 & -2.181978 \\
\hline $\mathrm{H}$ & 0.365477 & -0.673794 & -5.406641 \\
\hline $\mathrm{H}$ & 1.997697 & 1.185800 & -4.735774 \\
\hline $\mathrm{H}$ & -0.815803 & -1.436401 & -2.995193 \\
\hline $\mathrm{H}$ & 1.947809 & 1.639735 & -2.212543 \\
\hline C & -2.379794 & -2.295560 & 0.227422 \\
\hline C & -2.191728 & 2.358374 & -1.052349 \\
\hline $\mathrm{C}$ & 1.972936 & 2.194237 & 1.382672 \\
\hline $\mathrm{C}$ & 2.418183 & -2.335751 & -0.211322 \\
\hline C & -2.778877 & -1.026345 & -0.183501 \\
\hline C & -4.138882 & -0.609199 & -0.465811 \\
\hline C & -4.064225 & 0.665170 & -0.986113 \\
\hline C & -2.663470 & 1.052629 & -0.945814 \\
\hline $\mathrm{C}$ & -5.178200 & 1.555231 & -1.435421 \\
\hline C & -5.351537 & -1.451275 & -0.230566 \\
\hline $\mathrm{C}$ & -0.978747 & 2.792393 & -0.505224 \\
\hline $\mathrm{C}$ & -0.691029 & 4.153197 & -0.105876 \\
\hline $\mathrm{C}$ & 0.414604 & 4.097534 & 0.733539 \\
\hline $\mathrm{C}$ & 0.835325 & 2.700650 & 0.761776 \\
\hline C & -1.478992 & 5.350406 & -0.523518 \\
\hline $\mathrm{C}$ & 1.107347 & 5.166320 & 1.429163 \\
\hline $\mathrm{C}$ & 0.644385 & 6.395469 & 1.733936 \\
\hline $\mathrm{C}$ & 2.550852 & 0.956923 & 1.072128 \\
\hline $\mathrm{C}$ & 3.899116 & 0.544279 & 1.383992 \\
\hline $\mathrm{C}$ & 4.089058 & -0.687392 & 0.764173 \\
\hline C & 2.799820 & -1.045375 & 0.175206 \\
\hline $\mathrm{C}$ & 4.861493 & 1.315427 & 2.225977 \\
\hline $\mathrm{C}$ & 5.264590 & -1.535350 & 0.719679 \\
\hline $\mathrm{C}$ & 6.545956 & -1.192109 & 0.964422 \\
\hline $\mathrm{C}$ & 1.101258 & -2.787678 & -0.272517 \\
\hline $\mathrm{C}$ & 0.693928 & -4.180310 & -0.123480 \\
\hline C & -0.651459 & -4.163824 & 0.165040 \\
\hline C & -1.070849 & -2.771253 & 0.107463 \\
\hline C & 1.621014 & -5.350557 & -0.196147 \\
\hline $\mathrm{C}$ & -1.557903 & -5.312253 & 0.472886 \\
\hline $\mathrm{N}$ & -1.901164 & -0.007693 & -0.507339 \\
\hline $\mathrm{N}$ & -0.015393 & 1.942002 & -0.017077 \\
\hline $\mathrm{N}$ & 1.924850 & -0.003708 & 0.314122 \\
\hline $\mathrm{N}$ & -0.001002 & -1.971929 & -0.193052 \\
\hline $\mathrm{Fe}$ & 0.001162 & -0.003032 & -0.167830 \\
\hline $\mathrm{H}$ & -3.158633 & -3.012892 & 0.487865 \\
\hline $\mathrm{H}$ & -2.899916 & 3.121729 & -1.375302 \\
\hline $\mathrm{H}$ & 2.547427 & 2.871147 & 2.014470 \\
\hline $\mathrm{H}$ & 3.197725 & -3.097104 & -0.255922 \\
\hline $\mathrm{H}$ & -6.133317 & 1.014775 & -1.470673 \\
\hline $\mathrm{H}$ & -4.991785 & 1.964846 & -2.440633 \\
\hline $\mathrm{H}$ & -5.311139 & 2.412651 & -0.755326 \\
\hline $\mathrm{H}$ & -5.458363 & -1.712324 & 0.834297 \\
\hline $\mathrm{H}$ & -5.308687 & -2.397505 & -0.792861 \\
\hline
\end{tabular}




\begin{tabular}{|c|c|c|c|}
\hline $\mathrm{H}$ & & & 5 \\
\hline $\mathrm{H}$ & & & \\
\hline & -2.217838 & 5.645090 & 0.241154 \\
\hline $\mathrm{H}$ & -2.027189 & 5.169016 & -1.458909 \\
\hline $\mathrm{H}$ & -0.817135 & 6.215396 & -0.680344 \\
\hline $\mathrm{H}$ & 2.127732 & 4.931090 & 1.749714 \\
\hline $\mathrm{H}$ & -0.369440 & 6.722301 & 1.505170 \\
\hline $\mathrm{H}$ & 1.281033 & 7.110016 & 2.257516 \\
\hline $\mathrm{H}$ & 4.340091 & 1.994648 & 2.914567 \\
\hline $\mathrm{H}$ & 5.553310 & 1.924905 & 1.619826 \\
\hline $\mathrm{H}$ & 5.478602 & 0.634644 & 2.831242 \\
\hline $\mathrm{H}$ & 5.080381 & -2.577363 & 0.435788 \\
\hline $\mathrm{H}$ & 6.848308 & -0.176609 & 1.217474 \\
\hline $\mathrm{H}$ & 7.339592 & -1.937573 & 0.900100 \\
\hline $\mathrm{H}$ & 2.363602 & -5.332494 & 0.619334 \\
\hline $\mathrm{H}$ & 2.181048 & -5.370685 & -1.144596 \\
\hline $\mathrm{H}$ & 1.073538 & -6.298872 & -0.115327 \\
\hline $\mathrm{H}$ & -1.016847 & -6.267337 & 0.445343 \\
\hline $\mathrm{H}$ & -2.391477 & -5.384610 & -0.244764 \\
\hline $\mathrm{H}$ & -2.002717 & -5.215037 & 1.476164 \\
\hline C & -0.322520 & -0.167195 & 1.695602 \\
\hline $\mathrm{N}$ & -0.533004 & -0.265568 & 2.846030 \\
\hline lis $-\mathrm{H}$ & $\mathrm{CN}, 143.2^{\circ} \alpha-\mathrm{me}$ & $\mathrm{e}-\gamma$-meso angle & \\
\hline $\mathrm{C}$ & 0.506856 & -0.314012 & -4.407949 \\
\hline $\mathrm{N}$ & 1.407936 & 0.687566 & -4.094897 \\
\hline C & -0.066537 & -0.676455 & -3.214260 \\
\hline C & 1.356492 & 0.900981 & -2.752694 \\
\hline $\mathrm{N}$ & 0.469236 & 0.086664 & -2.196008 \\
\hline $\mathrm{H}$ & 0.362276 & -0.667738 & -5.421831 \\
\hline $\mathrm{H}$ & 2.007632 & 1.179471 & -4.748835 \\
\hline $\mathrm{H}$ & -0.821022 & -1.427912 & -3.010650 \\
\hline $\mathrm{H}$ & 1.960327 & 1.631449 & -2.225202 \\
\hline C & -2.373116 & -2.286686 & 0.241437 \\
\hline $\mathrm{C}$ & -2.186371 & 2.357495 & -1.069480 \\
\hline C & 1.962185 & 2.184785 & 1.390116 \\
\hline $\mathrm{C}$ & 2.420689 & -2.333429 & -0.229397 \\
\hline $\mathrm{C}$ & -2.773948 & -1.020847 & -0.179267 \\
\hline $\mathrm{C}$ & -4.134783 & -0.603450 & -0.457671 \\
\hline $\mathrm{C}$ & -4.060893 & 0.666264 & -0.989659 \\
\hline $\mathrm{C}$ & -2.659296 & 1.052197 & -0.959329 \\
\hline $\mathrm{C}$ & -5.175926 & 1.554268 & -1.440468 \\
\hline $\mathrm{C}$ & -5.347578 & -1.441294 & -0.208504 \\
\hline $\mathrm{C}$ & -0.974356 & 2.791832 & -0.519423 \\
\hline C & -0.690476 & 4.151009 & -0.111579 \\
\hline $\mathrm{C}$ & 0.408245 & 4.091988 & 0.736930 \\
\hline $\mathrm{C}$ & 0.830146 & 2.695356 & 0.761694 \\
\hline C & -1.476311 & 5.349596 & -0.529314 \\
\hline $\mathrm{C}$ & 1.094295 & 5.157763 & 1.443644 \\
\hline C & 0.629295 & 6.386326 & 1.747798 \\
\hline $\mathrm{C}$ & 2.545600 & 0.951590 & 1.072202 \\
\hline C & 3.892869 & 0.538205 & 1.387482 \\
\hline $\mathrm{C}$ & 4.088154 & -0.687944 & 0.758083 \\
\hline
\end{tabular}




\begin{tabular}{|c|c|c|c|}
\hline $\mathrm{C}$ & 2.802115 & -1.043582 & 0.160329 \\
\hline $\mathrm{C}$ & 4.849949 & 1.303833 & 2.240415 \\
\hline C & 5.265106 & -1.533712 & 0.711817 \\
\hline $\mathrm{C}$ & 6.544652 & -1.191897 & 0.967976 \\
\hline C & 1.103465 & -2.785842 & -0.286769 \\
\hline $\mathrm{C}$ & 0.696023 & -4.177424 & -0.127721 \\
\hline C & -0.646784 & -4.158199 & 0.173034 \\
\hline C & -1.065955 & -2.765715 & 0.111782 \\
\hline $\mathrm{C}$ & 1.621404 & -5.348926 & -0.202255 \\
\hline C & -1.551273 & -5.304307 & 0.495027 \\
\hline $\mathrm{N}$ & -1.896790 & -0.006690 & -0.517858 \\
\hline $\mathrm{N}$ & -0.013024 & 1.940717 & -0.029001 \\
\hline $\mathrm{N}$ & 1.925852 & -0.003547 & 0.302137 \\
\hline $\mathrm{N}$ & 0.001939 & -1.969465 & -0.203589 \\
\hline $\mathrm{Fe}$ & 0.004871 & -0.002651 & -0.183305 \\
\hline $\mathrm{H}$ & -3.150648 & -3.000613 & 0.514676 \\
\hline $\mathrm{H}$ & -2.894475 & 3.120847 & -1.392704 \\
\hline $\mathrm{H}$ & 2.530375 & 2.857475 & 2.032060 \\
\hline $\mathrm{H}$ & 3.200230 & -3.094797 & -0.274479 \\
\hline $\mathrm{H}$ & -6.132349 & 1.015427 & -1.464257 \\
\hline $\mathrm{H}$ & -4.995128 & 1.953677 & -2.450779 \\
\hline $\mathrm{H}$ & -5.302756 & 2.418543 & -0.767953 \\
\hline $\mathrm{H}$ & -5.445910 & -1.697468 & 0.858360 \\
\hline $\mathrm{H}$ & -5.311985 & -2.390037 & -0.767062 \\
\hline $\mathrm{H}$ & -6.264887 & -0.917167 & -0.507216 \\
\hline $\mathrm{H}$ & -2.218200 & 5.642609 & 0.233051 \\
\hline $\mathrm{H}$ & -2.020500 & 5.170883 & -1.467538 \\
\hline $\mathrm{H}$ & -0.813552 & 6.214808 & -0.681176 \\
\hline $\mathrm{H}$ & 2.110961 & 4.920430 & 1.774256 \\
\hline $\mathrm{H}$ & -0.381562 & 6.715216 & 1.509117 \\
\hline $\mathrm{H}$ & 1.261034 & 7.098267 & 2.280808 \\
\hline $\mathrm{H}$ & 4.324284 & 1.978184 & 2.930537 \\
\hline $\mathrm{H}$ & 5.545066 & 1.917728 & 1.642536 \\
\hline $\mathrm{H}$ & 5.463753 & 0.619276 & 2.844795 \\
\hline $\mathrm{H}$ & 5.083901 & -2.572717 & 0.415242 \\
\hline $\mathrm{H}$ & 6.844587 & -0.179095 & 1.234189 \\
\hline $\mathrm{H}$ & 7.339352 & -1.935907 & 0.899960 \\
\hline $\mathrm{H}$ & 2.370933 & -5.327430 & 0.606776 \\
\hline $\mathrm{H}$ & 2.173304 & -5.374358 & -1.155298 \\
\hline $\mathrm{H}$ & 1.073651 & -6.296237 & -0.111931 \\
\hline $\mathrm{H}$ & -1.010254 & -6.259534 & 0.471986 \\
\hline $\mathrm{H}$ & -2.388875 & -5.382205 & -0.217290 \\
\hline $\mathrm{H}$ & -1.990312 & -5.199177 & 1.500036 \\
\hline $\mathrm{C}$ & -0.320894 & -0.164107 & 1.680607 \\
\hline $\mathrm{N}$ & -0.532344 & -0.261124 & 2.830946 \\
\hline \multicolumn{4}{|c|}{ His-heme-CN, $142.2^{\circ} \alpha$-meso-Fe- $\gamma$-meso angle } \\
\hline $\mathrm{C}$ & 0.506435 & -0.311126 & -4.422086 \\
\hline $\mathrm{N}$ & 1.413921 & 0.684334 & -4.108260 \\
\hline $\mathrm{C}$ & -0.069969 & -0.670155 & -3.228809 \\
\hline $\mathrm{C}$ & 1.364887 & 0.896000 & -2.765623 \\
\hline $\mathrm{N}$ & 0.472069 & 0.087282 & -2.209549 \\
\hline
\end{tabular}




\begin{tabular}{|c|c|c|c|}
\hline $\mathrm{H}$ & 0.359256 & -0.662892 & -5.436272 \\
\hline $\mathrm{H}$ & 2.016730 & 1.172963 & -4.761774 \\
\hline $\mathrm{H}$ & -0.830280 & -1.415931 & -3.026043 \\
\hline $\mathrm{H}$ & 1.974389 & 1.621245 & -2.237471 \\
\hline $\mathrm{C}$ & -2.366489 & -2.278084 & 0.255757 \\
\hline $\mathrm{C}$ & -2.180442 & 2.356059 & -1.086385 \\
\hline $\mathrm{C}$ & 1.951717 & 2.175445 & 1.398023 \\
\hline $\mathrm{C}$ & 2.423064 & -2.330809 & -0.247216 \\
\hline $\mathrm{C}$ & -2.768903 & -1.015615 & -0.174668 \\
\hline C & -4.130435 & -0.597594 & -0.449476 \\
\hline $\mathrm{C}$ & -4.057083 & 0.667313 & -0.993183 \\
\hline $\mathrm{C}$ & -2.654636 & 1.051370 & -0.972586 \\
\hline C & -5.172930 & 1.553465 & -1.445691 \\
\hline $\mathrm{C}$ & -5.343408 & -1.431054 & -0.186829 \\
\hline $\mathrm{C}$ & -0.969562 & 2.790821 & -0.533352 \\
\hline $\mathrm{C}$ & -0.689881 & 4.148416 & -0.117262 \\
\hline C & 0.401679 & 4.086273 & 0.740582 \\
\hline C & 0.825142 & 2.689996 & 0.762179 \\
\hline C & -1.473699 & 5.348215 & -0.535364 \\
\hline $\mathrm{C}$ & 1.080496 & 5.149112 & 1.458511 \\
\hline $\mathrm{C}$ & 0.613720 & 6.377529 & 1.760695 \\
\hline $\mathrm{C}$ & 2.540339 & 0.946363 & 1.072666 \\
\hline $\mathrm{C}$ & 3.886611 & 0.531951 & 1.391006 \\
\hline $\mathrm{C}$ & 4.087123 & -0.688537 & 0.751989 \\
\hline C & 2.804228 & -1.041622 & 0.145675 \\
\hline $\mathrm{C}$ & 4.838587 & 1.291886 & 2.254625 \\
\hline C & 5.265447 & -1.532177 & 0.703692 \\
\hline C & 6.543175 & -1.192036 & 0.971034 \\
\hline C & 1.105660 & -2.783646 & -0.300613 \\
\hline C & 0.698386 & -4.174151 & -0.131845 \\
\hline $\mathrm{C}$ & -0.641724 & -4.152362 & 0.181179 \\
\hline $\mathrm{C}$ & -1.061066 & -2.760085 & 0.116615 \\
\hline $\mathrm{C}$ & 1.622172 & -5.346811 & -0.208533 \\
\hline $\mathrm{C}$ & -1.544061 & -5.296319 & 0.516692 \\
\hline $\mathrm{N}$ & -1.892120 & -0.006287 & -0.528026 \\
\hline $\mathrm{N}$ & -0.010104 & 1.939189 & -0.040434 \\
\hline $\mathrm{N}$ & 1.926665 & -0.003110 & 0.290396 \\
\hline $\mathrm{N}$ & 0.004730 & -1.966652 & -0.213585 \\
\hline $\mathrm{Fe}$ & 0.008725 & -0.002427 & -0.198405 \\
\hline $\mathrm{H}$ & -3.142567 & -2.988537 & 0.541812 \\
\hline $\mathrm{H}$ & -2.888528 & 3.119441 & -1.409746 \\
\hline $\mathrm{H}$ & 2.513408 & 2.843671 & 2.050302 \\
\hline $\mathrm{H}$ & 3.202608 & -3.092237 & -0.292575 \\
\hline $\mathrm{H}$ & -6.130650 & 1.016484 & -1.458434 \\
\hline $\mathrm{H}$ & -4.997423 & 1.942748 & -2.460860 \\
\hline $\mathrm{H}$ & -5.293761 & 2.424415 & -0.780758 \\
\hline $\mathrm{H}$ & -5.432440 & -1.684144 & 0.881581 \\
\hline $\mathrm{H}$ & -5.315820 & -2.381352 & -0.743219 \\
\hline $\mathrm{H}$ & -6.261882 & -0.904857 & -0.478271 \\
\hline $\mathrm{H}$ & -2.217806 & 5.640400 & 0.225161 \\
\hline $\mathrm{H}$ & -2.014805 & 5.171521 & -1.475722 \\
\hline $\mathrm{H}$ & -0.809993 & 6.213367 & -0.683640 \\
\hline
\end{tabular}




\begin{tabular}{|c|c|c|c|}
\hline & & & \\
\hline $\mathrm{H}$ & 2.092763 & 4.909285 & 1.800578 \\
\hline $\mathrm{H}$ & -0.393503 & 6.708968 & 1.510520 \\
\hline $\mathrm{H}$ & 1.240118 & 7.086783 & 2.303520 \\
\hline $\mathrm{H}$ & 4.308830 & 1.961576 & 2.946146 \\
\hline $\mathrm{H}$ & 5.537025 & 1.909906 & 1.664893 \\
\hline $\mathrm{H}$ & 5.449013 & 0.603534 & 2.858136 \\
\hline $\mathrm{H}$ & 5.087125 & -2.568007 & 0.394524 \\
\hline $\mathrm{H}$ & 6.840761 & -0.182104 & 1.250349 \\
\hline $\mathrm{H}$ & 7.338884 & -1.934607 & 0.899122 \\
\hline $\mathrm{H}$ & 2.377954 & -5.322568 & 0.594598 \\
\hline $\mathrm{H}$ & 2.166649 & -5.376651 & -1.165671 \\
\hline $\mathrm{H}$ & 1.074210 & -6.293193 & -0.109995 \\
\hline $\mathrm{H}$ & -1.002691 & -6.251458 & 0.498938 \\
\hline $\mathrm{H}$ & -2.384892 & -5.380117 & -0.191117 \\
\hline $\mathrm{H}$ & -1.978410 & -5.183146 & 1.522850 \\
\hline C & -0.319969 & -0.160309 & 1.666010 \\
\hline $\mathrm{N}$ & -0.532799 & -0.255521 & 2.816234 \\
\hline His- $\mathrm{r}$ & $\mathrm{N}, 141.2^{\circ} \alpha-\mathrm{m}$ & $\mathrm{e}-\gamma$-meso angle & \\
\hline $\mathrm{C}$ & 0.507530 & -0.308637 & -4.437339 \\
\hline $\mathrm{N}$ & 1.420793 & 0.681169 & -4.122629 \\
\hline C & -0.069841 & -0.666510 & -3.244154 \\
\hline $\mathrm{C}$ & 1.372578 & 0.892318 & -2.779794 \\
\hline $\mathrm{N}$ & 0.475684 & 0.087670 & -2.224296 \\
\hline $\mathrm{H}$ & 0.358102 & -0.658466 & -5.451863 \\
\hline $\mathrm{H}$ & 2.026314 & 1.166986 & -4.775715 \\
\hline $\mathrm{H}$ & -0.833815 & -1.408690 & -3.041958 \\
\hline $\mathrm{H}$ & 1.985941 & 1.613878 & -2.251076 \\
\hline C & -2.358984 & -2.268390 & 0.268975 \\
\hline C & -2.175786 & 2.355775 & -1.103117 \\
\hline C & 1.940556 & 2.165470 & 1.404049 \\
\hline C & 2.425884 & -2.329161 & -0.264640 \\
\hline C & -2.763590 & -1.009517 & -0.171122 \\
\hline $\mathrm{C}$ & -4.126081 & -0.591536 & -0.441435 \\
\hline C & -4.054112 & 0.668736 & -0.996250 \\
\hline C & -2.650958 & 1.051350 & -0.985982 \\
\hline C & -5.171411 & 1.552740 & -1.449378 \\
\hline C & -5.338669 & -1.421080 & -0.165007 \\
\hline C & -0.965489 & 2.790745 & -0.547636 \\
\hline C & -0.689598 & 4.146553 & -0.122769 \\
\hline $\mathrm{C}$ & 0.395284 & 4.080641 & 0.743443 \\
\hline C & 0.819765 & 2.684577 & 0.760904 \\
\hline $\mathrm{C}$ & -1.471499 & 5.347869 & -0.540109 \\
\hline C & 1.067595 & 5.140023 & 1.472457 \\
\hline C & 0.598869 & 6.367581 & 1.775228 \\
\hline $\mathrm{C}$ & 2.535020 & 0.940723 & 1.071807 \\
\hline C & 3.880244 & 0.525863 & 1.394024 \\
\hline C & 4.086248 & -0.689337 & 0.746401 \\
\hline C & 2.806777 & -1.040258 & 0.131204 \\
\hline $\mathrm{C}$ & 4.826697 & 1.280527 & 2.268227 \\
\hline $\mathrm{C}$ & 5.266006 & -1.530832 & 0.697608 \\
\hline $\mathrm{C}$ & 6.541888 & -1.191398 & 0.974619 \\
\hline
\end{tabular}




\begin{tabular}{|c|c|c|c|}
\hline $\mathrm{C}$ & 1.108042 & -2.782328 & -0.314608 \\
\hline $\mathrm{C}$ & 0.700375 & -4.171593 & -0.135654 \\
\hline $\mathrm{C}$ & -0.637073 & -4.146528 & 0.188826 \\
\hline $\mathrm{C}$ & -1.055705 & -2.754250 & 0.120232 \\
\hline C & 1.622212 & -5.345734 & -0.213182 \\
\hline C & -1.537784 & -5.287659 & 0.538053 \\
\hline $\mathrm{N}$ & -1.887924 & -0.004750 & -0.538964 \\
\hline $\mathrm{N}$ & -0.008020 & 1.938208 & -0.053069 \\
\hline $\mathrm{N}$ & 1.927862 & -0.003502 & 0.278289 \\
\hline $\mathrm{N}$ & 0.008157 & -1.964425 & -0.224279 \\
\hline $\mathrm{Fe}$ & 0.012597 & -0.002099 & -0.214520 \\
\hline $\mathrm{H}$ & -3.133622 & -2.975286 & 0.567255 \\
\hline $\mathrm{H}$ & -2.883922 & 3.119136 & -1.426390 \\
\hline $\mathrm{H}$ & 2.495894 & 2.829382 & 2.066070 \\
\hline $\mathrm{H}$ & 3.205388 & -3.090595 & -0.310313 \\
\hline $\mathrm{H}$ & -6.129904 & 1.016924 & -1.451956 \\
\hline $\mathrm{H}$ & -5.001449 & 1.933380 & -2.468746 \\
\hline $\mathrm{H}$ & -5.287138 & 2.429314 & -0.790981 \\
\hline $\mathrm{H}$ & -5.419072 & -1.669510 & 0.905177 \\
\hline $\mathrm{H}$ & -5.317914 & -2.373723 & -0.717667 \\
\hline $\mathrm{H}$ & -6.258413 & -0.893945 & -0.450755 \\
\hline $\mathrm{H}$ & -2.218729 & 5.637713 & 0.218243 \\
\hline $\mathrm{H}$ & -2.008505 & 5.174463 & -1.483412 \\
\hline $\mathrm{H}$ & -0.807073 & 6.213428 & -0.682759 \\
\hline $\mathrm{H}$ & 2.076259 & 4.897996 & 1.823460 \\
\hline $\mathrm{H}$ & -0.405463 & 6.701106 & 1.516408 \\
\hline $\mathrm{H}$ & 1.220482 & 7.073893 & 2.327321 \\
\hline $\mathrm{H}$ & 4.292602 & 1.944825 & 2.961599 \\
\hline $\mathrm{H}$ & 5.527669 & 1.903432 & 1.686681 \\
\hline $\mathrm{H}$ & 5.434570 & 0.588607 & 2.870232 \\
\hline $\mathrm{H}$ & 5.090797 & -2.564183 & 0.378493 \\
\hline $\mathrm{H}$ & 6.836855 & -0.183582 & 1.264016 \\
\hline $\mathrm{H}$ & 7.338853 & -1.932412 & 0.900497 \\
\hline $\mathrm{H}$ & 2.383590 & -5.318674 & 0.584556 \\
\hline $\mathrm{H}$ & 2.159964 & -5.380723 & -1.173907 \\
\hline $\mathrm{H}$ & 1.073701 & -6.290911 & -0.106372 \\
\hline $\mathrm{H}$ & -0.996945 & -6.243164 & 0.524012 \\
\hline $\mathrm{H}$ & -2.382822 & -5.375987 & -0.164111 \\
\hline $\mathrm{H}$ & -1.965980 & -5.166970 & 1.545959 \\
\hline $\mathrm{C}$ & -0.318262 & -0.156995 & 1.650357 \\
\hline $\mathrm{N}$ & -0.532127 & -0.250634 & 2.800478 \\
\hline \multicolumn{4}{|c|}{ His-heme-CN, $140.2^{\circ} \alpha$-meso-Fe- $\gamma$-meso angle } \\
\hline $\mathrm{C}$ & 0.507880 & -0.306148 & -4.451295 \\
\hline $\mathrm{N}$ & 1.427076 & 0.677822 & -4.135846 \\
\hline $\mathrm{C}$ & -0.072389 & -0.660719 & -3.258518 \\
\hline $\mathrm{C}$ & 1.380880 & 0.887475 & -2.792626 \\
\hline $\mathrm{N}$ & 0.478761 & 0.088193 & -2.237709 \\
\hline $\mathrm{H}$ & 0.356149 & -0.654201 & -5.466085 \\
\hline $\mathrm{H}$ & 2.035587 & 1.160441 & -4.788503 \\
\hline $\mathrm{H}$ & -0.841780 & -1.397499 & -3.057175 \\
\hline $\mathrm{H}$ & 1.999426 & 1.604119 & -2.26329 \\
\hline
\end{tabular}




\begin{tabular}{|c|c|c|c|}
\hline $\mathrm{C}$ & -2.351866 & -2.259239 & 0.283225 \\
\hline $\mathrm{C}$ & -2.169689 & 2.354207 & -1.119959 \\
\hline $\mathrm{C}$ & 1.929959 & 2.155708 & 1.411353 \\
\hline $\mathrm{C}$ & 2.428230 & -2.326750 & -0.282128 \\
\hline $\mathrm{C}$ & -2.758289 & -1.003957 & -0.166689 \\
\hline $\mathrm{C}$ & -4.121477 & -0.585184 & -0.433253 \\
\hline $\mathrm{C}$ & -4.050185 & 0.670037 & -0.999740 \\
\hline C & -2.646225 & 1.050459 & -0.999460 \\
\hline C & -5.168299 & 1.552299 & -1.454329 \\
\hline C & -5.334063 & -1.410165 & -0.143460 \\
\hline C & -0.960473 & 2.789739 & -0.561751 \\
\hline $\mathrm{C}$ & -0.689166 & 4.143842 & -0.128395 \\
\hline C & 0.388397 & 4.074649 & 0.747007 \\
\hline $\mathrm{C}$ & 0.814700 & 2.679025 & 0.761022 \\
\hline $\mathrm{C}$ & -1.469252 & 5.346331 & -0.545842 \\
\hline $\mathrm{C}$ & 1.053093 & 5.130878 & 1.487390 \\
\hline $\mathrm{C}$ & 0.582413 & 6.358111 & 1.788606 \\
\hline C & 2.529741 & 0.935263 & 1.071933 \\
\hline C & 3.873946 & 0.519304 & 1.397160 \\
\hline $\mathrm{C}$ & 4.085160 & -0.690192 & 0.740343 \\
\hline $\mathrm{C}$ & 2.808936 & -1.038501 & 0.116652 \\
\hline $\mathrm{C}$ & 4.815316 & 1.268200 & 2.281705 \\
\hline $\mathrm{C}$ & 5.266263 & -1.529654 & 0.690059 \\
\hline $\mathrm{C}$ & 6.540414 & -1.191350 & 0.976304 \\
\hline C & 1.110270 & -2.780315 & -0.328202 \\
\hline C & 0.702736 & -4.168304 & -0.139357 \\
\hline $\mathrm{C}$ & -0.631832 & -4.140394 & 0.197158 \\
\hline $\mathrm{C}$ & -1.050613 & -2.748356 & 0.125206 \\
\hline $\mathrm{C}$ & 1.622854 & -5.343712 & -0.218672 \\
\hline $\mathrm{C}$ & -1.530376 & -5.279172 & 0.559510 \\
\hline $\mathrm{N}$ & -1.883215 & -0.004538 & -0.549531 \\
\hline $\mathrm{N}$ & -0.004706 & 1.936774 & -0.064905 \\
\hline $\mathrm{N}$ & 1.928731 & -0.003221 & 0.266422 \\
\hline $\mathrm{N}$ & 0.011051 & -1.961612 & -0.234200 \\
\hline $\mathrm{Fe}$ & 0.016592 & -0.002111 & -0.229613 \\
\hline $\mathrm{H}$ & -3.124884 & -2.962383 & 0.594283 \\
\hline $\mathrm{H}$ & -2.877934 & 3.117650 & -1.443028 \\
\hline $\mathrm{H}$ & 2.478524 & 2.814881 & 2.083724 \\
\hline $\mathrm{H}$ & 3.207741 & -3.088274 & -0.327838 \\
\hline $\mathrm{H}$ & -6.127771 & 1.018210 & -1.447149 \\
\hline $\mathrm{H}$ & -5.003146 & 1.923901 & -2.477800 \\
\hline $\mathrm{H}$ & -5.278870 & 2.434650 & -0.802823 \\
\hline $\mathrm{H}$ & -5.405220 & -1.655576 & 0.928066 \\
\hline $\mathrm{H}$ & -5.321195 & -2.364282 & -0.693843 \\
\hline $\mathrm{H}$ & -6.254801 & -0.880897 & -0.422018 \\
\hline $\mathrm{H}$ & -2.218793 & 5.635050 & 0.210657 \\
\hline $\mathrm{H}$ & -2.003046 & 5.175053 & -1.491326 \\
\hline $\mathrm{H}$ & -0.804071 & 6.211977 & -0.684668 \\
\hline $\mathrm{H}$ & 2.057140 & 4.886277 & 1.849637 \\
\hline $\mathrm{H}$ & -0.418105 & 6.694126 & 1.518541 \\
\hline $\mathrm{H}$ & 1.198307 & 7.061591 & 2.350650 \\
\hline $\mathrm{H}$ & 4.277150 & 1.926996 & 2.977177 \\
\hline
\end{tabular}




$\begin{array}{rrrr}\mathrm{H} & 5.518630 & 1.895988 & 1.708253 \\ \mathrm{H} & 5.420782 & 0.572503 & 2.881795 \\ \mathrm{H} & 5.093796 & -2.560266 & 0.360727 \\ \mathrm{H} & 6.833002 & -0.185913 & 1.276161 \\ \mathrm{H} & 7.338502 & -1.930856 & 0.899187 \\ \mathrm{H} & 2.389326 & -5.314706 & 0.574126 \\ \mathrm{H} & 2.154424 & -5.382443 & -1.182646 \\ \mathrm{H} & 1.073975 & -6.287900 & -0.105138 \\ \mathrm{H} & -0.989518 & -6.234715 & 0.549506 \\ \mathrm{H} & -2.379068 & -5.372289 & -0.137581 \\ \mathrm{H} & -1.953170 & -5.151151 & 1.568774 \\ \mathrm{C} & -0.318261 & -0.152291 & 1.635693 \\ \mathrm{~N} & -0.534143 & -0.243462 & 2.785633\end{array}$

His-heme-CN, $139.1^{\circ} \alpha$-meso-Fe- $\gamma$-meso angle

$\begin{array}{lrrr}\mathrm{C} & 0.508390 & -0.303289 & -4.465301 \\ \mathrm{~N} & 1.433399 & 0.674844 & -4.149012 \\ \mathrm{C} & -0.073253 & -0.656204 & -3.272680 \\ \mathrm{C} & 1.388494 & 0.883535 & -2.805501 \\ \mathrm{~N} & 0.482079 & 0.088679 & -2.251096 \\ \mathrm{H} & 0.354259 & -0.649323 & -5.480416 \\ \mathrm{H} & 2.044627 & 1.154593 & -4.801222 \\ \mathrm{H} & -0.846828 & -1.388788 & -3.072075 \\ \mathrm{H} & 2.011302 & 1.596062 & -2.275639 \\ \mathrm{C} & -2.344489 & -2.249930 & 0.297384 \\ \mathrm{C} & -2.163857 & 2.352662 & -1.136311 \\ \mathrm{C} & 1.919194 & 2.145658 & 1.418228 \\ \mathrm{C} & 2.430636 & -2.324691 & -0.299412 \\ \mathrm{C} & -2.752869 & -0.998313 & -0.162333 \\ \mathrm{C} & -4.116756 & -0.578908 & -0.425149 \\ \mathrm{C} & -4.046345 & 0.671295 & -1.002922 \\ \mathrm{C} & -2.641627 & 1.049496 & -1.012563 \\ \mathrm{C} & -5.165399 & 1.551794 & -1.458658 \\ \mathrm{C} & -5.329082 & -1.399616 & -0.122368 \\ \mathrm{C} & -0.955502 & 2.788720 & -0.575586 \\ \mathrm{C} & -0.688792 & 4.141137 & -0.133848 \\ \mathrm{C} & 0.381635 & 4.068601 & 0.750295 \\ \mathrm{C} & 0.809527 & 2.673338 & 0.760821 \\ \mathrm{C} & -1.467201 & 5.344825 & -0.551014 \\ \mathrm{C} & 1.038933 & 5.121453 & 1.501908 \\ \mathrm{C} & 0.567117 & 6.348814 & 1.801032 \\ \mathrm{C} & 2.524179 & 0.929509 & 1.071923 \\ \mathrm{C} & 3.867424 & 0.512697 & 1.400142 \\ \mathrm{C} & 4.083941 & -0.691140 & 0.734502 \\ \mathrm{C} & 2.810998 & -1.036964 & 0.102446 \\ \mathrm{C} & 4.803631 & 1.256008 & 2.294809 \\ \mathrm{C} & 5.266473 & -1.528467 & 0.683164 \\ \mathrm{C} & 6.538857 & -1.190885 & 0.978043 \\ \mathrm{C} & 1.112430 & -2.778440 & -0.341545 \\ \mathrm{C} & 0.704927 & -4.165089 & -0.143012 \\ \mathrm{C} & -0.626680 & -4.134154 & 0.205258 \\ \mathrm{C} & -1.045374 & -2.742302 & 0.130224\end{array}$




$\begin{array}{lrrr}\mathrm{C} & 1.623219 & -5.341849 & -0.223782 \\ \mathrm{C} & -1.523208 & -5.270414 & 0.580374 \\ \mathrm{~N} & -1.878594 & -0.004365 & -0.559788 \\ \mathrm{~N} & -0.001536 & 1.935307 & -0.076557 \\ \mathrm{~N} & 1.929310 & -0.003471 & 0.254988 \\ \mathrm{~N} & 0.014113 & -1.958709 & -0.243571 \\ \mathrm{Fe} & 0.020677 & -0.002470 & -0.244770 \\ \mathrm{H} & -3.115800 & -2.949363 & 0.620646 \\ \mathrm{H} & -2.872240 & 3.116147 & -1.459069 \\ \mathrm{H} & 2.461189 & 2.800071 & 2.100475 \\ \mathrm{H} & 3.210121 & -3.086246 & -0.345452 \\ \mathrm{H} & -6.125718 & 1.019381 & -1.441818 \\ \mathrm{H} & -5.005231 & 1.914584 & -2.486065 \\ \mathrm{H} & -5.270925 & 2.439690 & -0.813907 \\ \mathrm{H} & -5.390114 & -1.643779 & 0.950050 \\ \mathrm{H} & -5.324648 & -2.354281 & -0.671971 \\ \mathrm{H} & -6.250900 & -0.867624 & -0.392094 \\ \mathrm{H} & -2.218153 & 5.632906 & 0.204331 \\ \mathrm{H} & -1.998839 & 5.175540 & -1.498041 \\ \mathrm{H} & -0.801180 & 6.210314 & -0.687040 \\ \mathrm{H} & 2.037882 & 4.873651 & 1.875852 \\ \mathrm{H} & -0.429055 & 6.688041 & 1.519277 \\ \mathrm{H} & 1.177426 & 7.049070 & 2.373114 \\ \mathrm{H} & 4.261383 & 1.909247 & 2.992340 \\ \mathrm{H} & 5.509076 & 1.888659 & 1.729341 \\ \mathrm{H} & 5.406892 & 0.556679 & 2.892900 \\ \mathrm{H} & 5.096911 & -2.556536 & 0.344477 \\ \mathrm{H} & 6.828882 & -0.187582 & 1.287308 \\ \mathrm{H} & 7.338224 & -1.928747 & 0.898394 \\ \mathrm{H} & 2.393856 & -5.311559 & 0.564947 \\ \mathrm{H} & 2.149677 & -5.383843 & -1.190381 \\ \mathrm{H} & 1.073725 & -6.285006 & -0.104759 \\ \mathrm{H} & -0.982443 & -6.226039 & 0.574379 \\ \mathrm{H} & -2.375398 & -5.368086 & -0.111777 \\ \mathrm{H} & -1.940791 & -5.135146 & 1.590843 \\ \mathrm{C} & -0.318962 & -0.147075 & 1.620920 \\ \mathrm{~N} & -0.537255 & -0.235283 & 2.770616 \\ & & & \end{array}$

